TRANSACTIONS OF THE

AMERICAN MATHEMATICAL SOCIETY

Volume 349, Number 1, January 1997, Pages 55-102

S 0002-9947(97)01634-6

\title{
EXPANSIVE SUBDYNAMICS
}

\author{
MIKE BOYLE AND DOUGLAS LIND
}

\begin{abstract}
This paper provides a framework for studying the dynamics of commuting homeomorphisms. Let $\alpha$ be a continuous action of $\mathbb{Z}^{d}$ on an infinite compact metric space. For each subspace $V$ of $\mathbb{R}^{d}$ we introduce a notion of expansiveness for $\alpha$ along $V$, and show that there are nonexpansive subspaces in every dimension $\leq d-1$. For each $k \leq d$ the set $\mathbb{E}_{k}(\alpha)$ of expansive $k$ dimensional subspaces is open in the Grassmann manifold of all $k$-dimensional subspaces of $\mathbb{R}^{d}$. Various dynamical properties of $\alpha$ are constant, or vary nicely, within a connected component of $\mathbb{E}_{k}(\alpha)$, but change abruptly when passing from one expansive component to another. We give several examples of this sort of "phase transition," including the topological and measure-theoretic directional entropies studied by Milnor, zeta functions, and dimension groups. For $d=2$ we show that, except for one unresolved case, every open set of directions whose complement is nonempty can arise as an $\mathbb{E}_{1}(\alpha)$. The unresolved case is that of the complement of a single irrational direction. Algebraic examples using commuting automorphisms of compact abelian groups are an important source of phenomena, and we study several instances in detail. We conclude with a set of problems and research directions suggested by our analysis.
\end{abstract}

\section{Contents}

1. Introduction

2. Definitions and examples

3. Nonexpansive subspaces

4. Realization

5. Regularity

6. Entropy

7. Algebraic examples

8. Markov subdynamics

9. Problems

References

Received by the editors May 6, 1994 .

1991 Mathematics Subject Classification. Primary 54H20, 58F03; Secondary 28D20, 28D15, 28F15, 58F $11,58 \mathrm{~F} 08$.

Key words and phrases. Expansive, subdynamics, symbolic dynamics, entropy, directional entropy, shift of finite type, group automorphism.

The first author was supported in part by NSF Grants DMS-8802593, DMS-9104134, and DMS-9401538.

The second author was supported in part by NSF Grants DMS-9004253 and DMS-9303240. 


\section{INTRODUCTION}

Expansiveness is a multifaceted dynamical condition which, in particular, plays an important role in the exploitation of hyperbolicity in smooth dynamical systems [Man2]. A homeomorphism $T$ of a compact metric space $(X, \rho)$ to itself is called expansive if there is a $\delta>0$ such that if $\rho\left(T^{n} x, T^{n} y\right) \leq \delta$ for all $n \in \mathbb{Z}$, then $x=y$. In other words, $T$ is expansive if, for each pair of distinct points, some iterate of $T$ separates them by a definite amount.

Let $\alpha$ denote a continuous action of $\mathbb{Z}^{d}$ on $(X, \rho)$. Thus $\alpha$ is generated by $d$ commuting homeomorphisms. There is an obvious extension of the notion of expansiveness to such actions (see $\S 2$ ). To avoid trivial exceptions, we will assume throughout that $X$ is infinite. Such actions occur in the study of smooth dynamics [KaSp], symbolic dynamics [N2], cellular automata [Mi], and automorphisms of compact groups [KS1]. It is natural to study $\alpha$ by considering those actions induced by subgroups of $\mathbb{Z}^{d}$ which are expansive. Crucial to this approach is considering expansiveness (in the sense of Definition 2.2) not just for subgroups of $\mathbb{Z}^{d}$, but for general subsets of $\mathbb{R}^{d}$.

We consider the "subdynamics" of an expansive $\mathbb{Z}^{d}$-action $\alpha$ along a subset of $\mathbb{R}^{d}$ by looking at the action of elements of $\mathbb{Z}^{d}$ which lie within a bounded distance of the subset. This leads to a natural notion of $\alpha$ being expansive along a subset of $\mathbb{R}^{d}$ (the exact definition is in $\S 2$ ). This notion generalizes the usual one: if the subset is a subgroup $H$ of $\mathbb{Z}^{d}$, then the action of $H$ induced by $\alpha$ is expansive if and only if $\alpha$ is expansive along $H$.

We study especially expansiveness along linear subspaces of $\mathbb{R}^{d}$. Let $\mathbb{G}_{k}$ denote the compact Grassmann manifold of $k$-dimensional subspaces (or $k$-planes) of $\mathbb{R}^{d}$, and let $\mathbb{N}_{k}(\alpha)$ denote the set of $k$-planes which are nonexpansive for $\alpha$. In $\S 3$ we prove our main structure theorem, that $\mathbb{N}_{d-1}(\alpha)$ is a nonempty closed subset of $\mathbb{G}_{d-1}$ which determines the lower-dimensional expansive subdynamics, as follows: a $k$-plane is nonexpansive if and only if it is contained in a nonexpansive $(d-1)$ plane. In $\S 4$ we investigate the question of which compact subsets of $\mathbb{G}_{d-1}$ can arise as the nonexpansive set of a $\mathbb{Z}^{d}$-action. In particular we show that when $d=2$ every nonempty compact set of lines can be an $\mathbb{N}_{1}(\alpha)$, with the sole unresolved possibility being a singleton set that contains just one irrational line.

Expansiveness can be viewed as a regularity condition on the subdynamics as the subspace varies. If we consider $k$-frames (i.e. $k$-tuples of linearly independent vectors) spanning $k$-planes, then sometimes a dynamical property of $k$-planes is constant or varies nicely within a connected component of the open set of $k$-frames for expansive $k$-planes, but this property changes abruptly when passing from one component to another. Heuristically, this is a "phase transition" for the action. We discuss this point of view in $\$ 5$ and give several examples. One reason it is essential to consider the subdynamics along general linear subspaces of $\mathbb{R}^{d}$ is that the boundaries of these components, though vividly reflected in the parameterization of dynamical properties of subactions of $\mathbb{Z}^{d}$, might not contain any nonzero integral vectors. This point was made explicitly by Katok and Spatzier [KaSp] in their work on invariant measures for smooth hyperbolic $\mathbb{Z}^{d}$-actions (see $\S 5$ ).

As a specific example of this philosophy, in $\S 6$ we extend work of Milnor to show in Theorem 6.16 that in an expansive component of $k$-frames, the $k$-dimensional measure theoretic directional entropy is given by a $k$-form. We prove in Theorem 6.13 a variational result for expansive frames, and show that for an expansive 
component of $k$-frames, the existence of a common measure of maximal entropy is equivalent to multilinearity of the $k$-dimensional topological directional entropy (Theorem 6.25). We describe explicitly in Theorem 6.33 the functions (nice but not necessarily linear) which can be the topological directional entropy of a $\mathbb{Z}^{2}$-action on a proper expansive component of 1 -frames.

In $\S 7$ we investigate the detailed behavior of a class of algebraically defined examples, and relate the lowest dimension of any expansive subspace to the Krull dimension of the quotient of a Laurent polynomial ring in several variables.

As another application of our regularity viewpoint, we show in $\S 8$ that in a connected component of expansive lines, either all elements have the Markov property or none do. In the Markov components we prove that the directional entropy varies linearly, and in the zero-dimensional case we parameterize the zeta functions and shift equivalence classes. We conclude in $\S 9$ with a discussion of open problems.

We began this work in 1989 as a response to a lecture [N1] of Nasu on his analysis [N2] of automorphisms of shifts as "textile systems." He studied especially certain cones of elements of $\mathbb{Z}^{2}$ which acted as expansive homeomorphisms, and without this influence our paper would not have been written. A crucial influence for us is Milnor's "entropy geometry" [Mi], the object of much of our paper and the background against which it was natural to consider expansiveness for general subsets of $\mathbb{R}^{d}$. We also thank Sam Lightwood for his careful reading and helpful comments on a preliminary version of this paper. About half of this work was written during the Fall, 1992, Program in Symbolic Dynamics at the Mathematical Sciences Research Institute in Berkeley, which provided a rich mathematical environment, natural beauty, warm and efficient staff, and excellent computer facilities. We are grateful for the pleasure of working in that ideal place.

\section{Definitions And EXAmples}

Let $(X, \rho)$ be a compact metric space, which we will always assume is infinite. A $\mathbb{Z}^{d}$-action $\alpha$ on $X$ is a homomorphism from the additive group $\mathbb{Z}^{d}$ to the group of homeomorphisms of $X$. For $\mathbf{n} \in \mathbb{Z}^{d}$, we denote the corresponding homeomorphism by $\alpha^{\mathbf{n}}$, so that $\alpha^{\mathbf{m}} \circ \alpha^{\mathbf{n}}=\alpha^{\mathbf{m}+\mathbf{n}}$ and $\alpha^{\mathbf{0}}$ is the identity on $X$. For a subset $F \subset \mathbb{R}^{d}$, put

If $F \cap \mathbb{Z}^{d}=\varnothing$, then put $\rho_{\alpha}^{F}(x, y)=0$.

$$
\rho_{\alpha}^{F}(x, y)=\sup \left\{\rho\left(\alpha^{\mathbf{n}}(x), \alpha^{\mathbf{n}}(y)\right): \mathbf{n} \in F \cap \mathbb{Z}^{d}\right\} .
$$

Definition 2.1. A $\mathbb{Z}^{d}$-action $\alpha$ on $X$ is expansive provided there is a $\delta>0$ such that $\rho_{\alpha}^{\mathbb{R}^{d}}(x, y) \leq \delta$ implies that $x=y$. In this case, $\delta$ is called an expansive constant for $\alpha$.

Suppose that $F$ is a subset of $\mathbb{R}^{d}$. Let $\|\cdot\|$ denote the Euclidean norm on $\mathbb{R}^{d}$, and for $\mathbf{v} \in \mathbb{R}^{d}$ define $\operatorname{dist}(\mathbf{v}, F)$ to be $\inf \{\|\mathbf{v}-\mathbf{w}\|: \mathbf{w} \in F\}$. For $t>0$ put $F^{t}=\left\{\mathbf{v} \in \mathbb{R}^{d}: \operatorname{dist}(\mathbf{v}, F) \leq t\right\}$, so that $F^{t}$ is the result of thickening $F$ by $t$.

Definition 2.2. Let $\alpha$ be a $\mathbb{Z}^{d}$-action on $X$, and $F$ be a subset of $\mathbb{R}^{d}$. Then $F$ is expansive for $\alpha$ if there are $\epsilon>0$ and $t>0$ such that $\rho_{\alpha}^{F^{t}}(x, y) \leq \epsilon$ implies that $x=y$. If $F$ fails to meet this condition, it is called nonexpansive for $\alpha$.

Every translate of an expansive set is also expansive. This follows from the observation that for $F \subset \mathbb{R}^{d}$ and $\mathbf{v} \in \mathbb{R}^{d}$,

$$
F^{t} \subset(F+\mathbf{v})^{t+|| \mathbf{v} \|} \text {. }
$$


The following result shows that by additional thickening, we can use the same $\epsilon$ for all expansive subsets of a given action.

Lemma 2.3. Let $\alpha$ be an expansive $\mathbb{Z}^{d}$-action on $X$ with expansive constant $\delta$. Then for every expansive subset $F$ of $\mathbb{R}^{d}$, there is an $s>0$ such that $\rho_{\alpha}^{F^{s}}(x, y) \leq \delta$ implies that $x=y$.

Proof. Let $F$ be an expansive subset for $\alpha$, so there are $\epsilon, t>0$ such that $\rho_{\alpha}^{F^{t}}(x, y) \leq$ $\epsilon$ implies that $x=y$. Let $B(r)$ denote $\left\{\mathbf{v} \in \mathbb{R}^{d}:\|\mathbf{v}\| \leq r\right\}$. Since $\alpha$ is expansive with constant $\delta$, a standard compactness argument shows that there is an $r$ such that $\rho_{\alpha}^{B(r)}(x, y) \leq \delta$ implies that $\rho(x, y) \leq \epsilon$. Put $s=t+r$.

Suppose that $x, y \in X$ are such that $\rho_{\alpha}^{F^{s}}(x, y) \leq \delta$. If $\mathbf{n} \in F^{t} \cap \mathbb{Z}^{d}$, then $\mathbf{n}+B(r) \subset F^{s}$, so that $\rho_{\alpha}^{B(r)}\left(\alpha^{\mathbf{n}} x, \alpha^{\mathbf{n}} y\right) \leq \delta$, and hence $\rho\left(\alpha^{\mathbf{n}} x, \alpha^{\mathbf{n}} y\right) \leq \epsilon$. Thus $\rho_{\alpha}^{F^{t}}(x, y) \leq \epsilon$, and so $x=y$.

It is convenient to have a term to describe the situation in the previous lemma.

Definition 2.4. Let $\alpha$ be a $\mathbb{Z}^{d}$-action with expansive constant $\delta$, and let $F$ be a subset of $\mathbb{R}^{d}$. A number $s>0$ is called an expansive radius for $F$ if $\rho_{\alpha}^{F^{s}}(x, y) \leq \delta$ implies that $x=y$.

In this terminology, Lemma 2.3 says that an expansive set has an expansive radius. For some actions there is a uniform $s$ which is an expansive radius for every expansive subset. We do not know if this holds for every action (see Problem 9.13).

From now on we shall only be concerned with subsets that are subspaces of $\mathbb{R}^{d}$. In order to discuss sets of subspaces, we recall the Grassmann manifold $\mathbb{G}_{k}=\mathbb{G}_{k, d}$ of all $k$-dimensional subspaces (or $k$-planes) of $\mathbb{R}^{d}$. See $[\mathrm{FR}, \S 3.2 .2]$ for an account of the properties of Grassmann manifolds used here. The topology of $\mathbb{G}_{k}$ is induced by the metric for which the distance between two subspaces is the Hausdorff metric distance between their intersections with the unit sphere in $\mathbb{R}^{d}$. Then $\mathbb{G}_{k}$ is a compact manifold of dimension $k(d-k)$. In particular, $\mathbb{G}_{1}$ is real projective $(d-$ $1)$-space. Also, a $k$-dimensional subspace is determined by its $(d-k)$-dimensional orthogonal complement, and this correspondence is a homeomorphism between $\mathbb{G}_{k}$ and $\mathbb{G}_{d-k}$.

Definition 2.5. For a $\mathbb{Z}^{d}$-action $\alpha$, define

$$
\begin{aligned}
& \mathbb{E}_{k}(\alpha)=\left\{V \in \mathbb{G}_{k}: V \text { is expansive for } \alpha\right\}, \\
& \mathbb{N}_{k}(\alpha)=\left\{V \in \mathbb{G}_{k}: V \text { is nonexpansive for } \alpha\right\} .
\end{aligned}
$$

We will show in the next section that $\mathbb{E}_{k}(\alpha)$ is always open in $\mathbb{G}_{k}$.

Remark 2.6. If $V$ is expansive for $\alpha$ and $W$ is a subspace containing $V$, then clearly $W$ is also expansive for $\alpha$. In particular, if $\alpha$ has an expansive subspace, then $\alpha$ itself must be expansive. Similarly, any subspace of a nonexpansive subspace is nonexpansive. If $\alpha$ itself is nonexpansive, then all subspaces of $\mathbb{R}^{d}$ are nonexpansive, so $\alpha$ has no expansive subdynamics. For this reason we are only interested in expansive $\mathbb{Z}^{d}$-actions.

Let us consider some instructive examples.

Example 2.7. Let $\mathcal{A}$ be a finite alphabet, and put $X=\mathcal{A}^{\mathbb{Z}^{d}}$, equipped with the product topology. Thus a point $x \in X$ has the form $\left(x(\mathbf{n}): \mathbf{n} \in \mathbb{Z}^{d}\right)$. To define a 


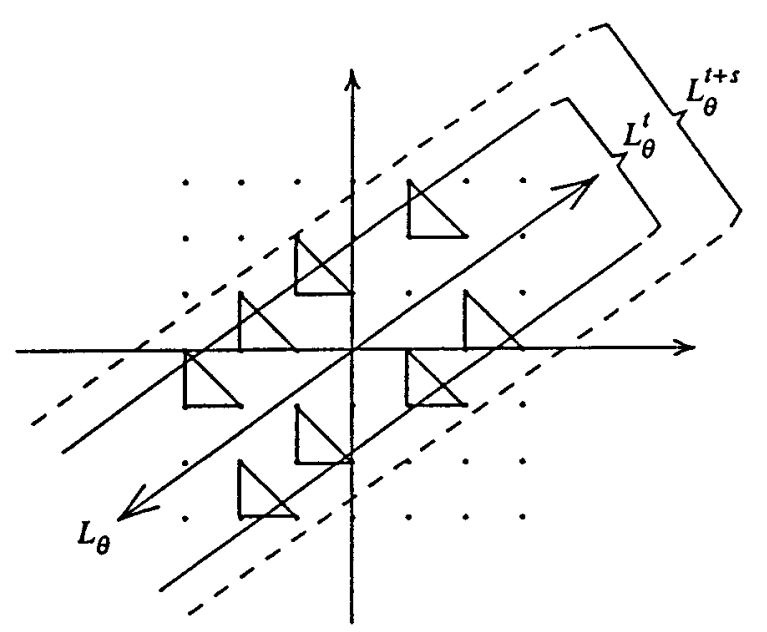

Figure 1. Coordinates in $L_{\theta}^{t}$ determine those in $L_{\theta}^{t+s}$.

metric on $X$, for $a, b \in \mathcal{A}$ let $\nu(a, b)=1$ if $a \neq b$ and 0 otherwise. Define $\rho$ on $X$ by

$$
\rho(x, y)=\sum_{\mathbf{n} \in \mathbb{Z}^{d}} 2^{-\|\mathbf{n}\|} \nu(x(\mathbf{n}), y(\mathbf{n})) .
$$

Two points are close in this metric provided their coordinates agree in a large neighborhood of the origin, so that $\rho$ induces the product topology on $X$. Thus we can recast the definition of expansiveness of $\alpha$ on $X$, or on any compact $\alpha$-invariant subset of $X$, as follows. A subspace $V$ is expansive if there is a $t$ such that if $x$ and $y$ agree on $V^{t}$ (i.e., $x(\mathbf{n})=y(\mathbf{n})$ for all $\mathbf{n} \in V^{t}$ ), then $x=y$.

Define the shift action $\alpha$ of $\mathbb{Z}^{d}$ on $X$ by $\left(\alpha^{\mathbf{n}} x\right)(\mathbf{k})=x(\mathbf{n}+\mathbf{k})$. Since $\rho(x, y)<1$ implies that $x(\mathbf{0})=y(\mathbf{0})$, it follows that $\alpha$ is expansive. However, no proper subspace $V$ of $\mathbb{R}^{d}$ can be expansive for $\alpha$, since, regardless of the size of $t$, there are distinct points in $X$ that agree on $V^{t}$. Thus $\mathbb{N}_{k}(\alpha)=\mathbb{G}_{k}$ for $0 \leq k \leq d-1$.

Example 2.8. Using the notation of Example 2.7, take $d=2, \mathcal{A}=\mathbb{Z} / 2 \mathbb{Z}$, and consider the compact $\alpha$-invariant subset $X$ of $(\mathbb{Z} / 2 \mathbb{Z})^{\mathbb{Z}^{2}}$ defined by the condition

$$
x(i, j)+x(i+1, j)+x(i, j+1) \equiv 0 \quad(\bmod 2)
$$

for all $i, j \in \mathbb{Z}$. This example is a subsystem of an expansive $\mathbb{Z}^{2}$-action, so is itself expansive. It is a simplified version of one studied by Ledrappier [Led], who showed that, as a Haar measure-preserving action, it is mixing but not mixing of higher orders.

To describe the expansive lines for $\alpha$, let $L_{\theta}$ denote the line making angle $\theta$ with the positive horizontal axis. Then $\mathbb{G}_{1}=\left\{L_{\theta}: 0 \leq \theta<\pi\right\}$. Let $\Delta$ be the triangle in $\mathbb{R}^{2}$ with vertices $\mathbf{0}=(0,0), \mathbf{e}_{1}=(1,0)$, and $\mathbf{e}_{2}=(0,1)$. Fix a $\theta \in[0, \pi)$ distinct from $0, \pi / 2$, and $3 \pi / 4$ (these exceptions come from the lines that are parallel to the faces of $\Delta$ ). Observe that there is an $s=s(\theta)>0$ with the following property. For all large enough $t$, each lattice point in $L_{\theta}^{t+s} \backslash L_{\theta}^{t}$ is the vertex of a translate of $\Delta$ whose other two vertices lie in $L_{\theta}^{t}$ (see Figure 1). Use of $(2-2)$ then shows that if $x$ and $y$ agree on $L_{\theta}^{t}$, then they agree on $L_{\theta}^{t+s}$. Repeating this observation shows 


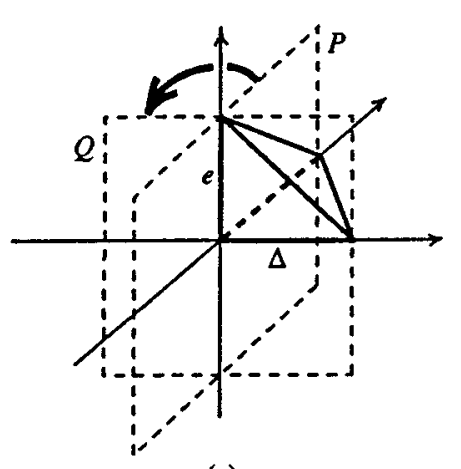

(a)

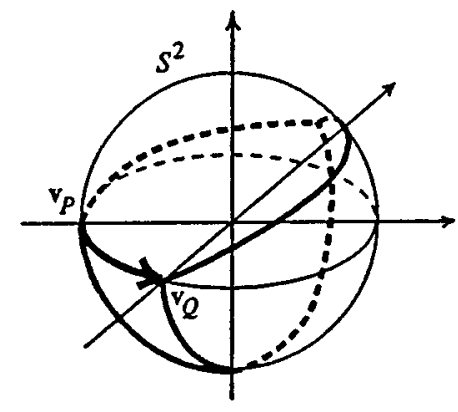

(b)

FiguRE 2. Description of the nonexpansive planes in Example 2.9.

that they must also agree on $L_{\theta}^{t+2 s}$, hence on $L_{\theta}^{t+3 s}$, and so on, so they must be equal. Thus $L_{\theta}$ is expansive.

However, if $L_{\theta}$ is parallel to a face of $\Delta$, then for every $t>0$ it is easy to construct a nonzero point that is 0 at all the coordinates in $L_{\theta}^{t}$. For example, if $\theta=0$, define $x(m, n)=0$ if $n \geq 0, x(0, n)=1$ if $n \leq-1$, and reconstruct the rest of $x$ using $(2-2)$. Translates of this point suffice to show that $L_{0}$ is not expansive.

Kitchens and Schmidt [KS2] compute the set of expansive lines for a class of such examples when $d=2$.

Example 2.9. We consider an analogue of Example 2.8 for $d=3$. Let $X$ be the set of points $x$ in $(\mathbb{Z} / 2 \mathbb{Z})^{\mathbb{Z}^{3}}$ satisfying

$$
x(i, j, k)+x(i+1, j, k)+x(i, j+1, k)+x(i, j, k+1) \equiv 0 \quad(\bmod 2)
$$

for all $i, j, k \in \mathbb{Z}$, and $\alpha$ be the shift action of $\mathbb{Z}^{3}$ on $X$. Let $\Delta$ denote the convex hull in $\mathbb{R}^{3}$ of $\mathbf{0}=(0,0,0), \mathbf{e}_{1}=(1,0,0), \mathbf{e}_{2}=(0,1,0)$, and $\mathbf{e}_{3}=(0,0,1)$. For reasons entirely analogous to the previous example, a plane is nonexpansive for $\alpha$ exactly when some translate of it is a support plane for $\Delta$ that contains an edge of $\Delta$, so this condition determines $\mathbb{N}_{2}(\alpha)$. To describe $\mathbb{N}_{1}(\alpha)$, note that every line in $\mathbb{R}^{3}$ can be translated to intersect an edge of $\Delta$ so that together they span a support plane. By the previous remark, the plane, hence this line, is nonexpansive. This shows that every line is nonexpansive, so that $\mathbb{N}_{1}(\alpha)=\mathbb{G}_{1}$. In other words, the union of the lines contained in the planes of $\mathbb{N}_{2}(\alpha)$ is all of $\mathbb{G}_{1}$.

Let us try to imagine what $\mathbb{N}_{2}(\alpha)$ looks like. We will do this by first using oriented planes, then dropping the orientation. An oriented plane is determined by its unit normal, so the space $\widetilde{\mathbb{G}}_{2}$ of oriented planes is homeomorphic to the space $\widetilde{\mathbb{G}}_{1}$ of oriented lines, which is homeomorphic to the 2 -sphere $S^{2}$. The maps $p_{2}: \widetilde{\mathbb{G}}_{2} \rightarrow \mathbb{G}_{2}$ and $p_{1}: \widetilde{\mathbb{G}}_{1} \rightarrow \mathbb{G}_{1}$ that forget orientation are 2 -to-1 covering maps that intertwine the homeomorphisms $\widetilde{\mathbb{G}}_{2} \cong \widetilde{\mathbb{G}}_{1}$ and $\mathbb{G}_{2} \cong \mathbb{G}_{1}$.

Each oriented plane $P \in \widetilde{\mathbb{G}}_{2}$ can be translated to a unique support plane for $\Delta$ with normal directed away from $\Delta$. Let $\widetilde{\mathbb{N}}_{2}(\alpha)$ denote the set of those $P$ for which the corresponding support plane contains an edge of $\Delta$. By our previous discussion, $\mathbb{N}_{2}(\alpha)=p_{2}\left(\widetilde{\mathbb{N}}_{2}(\alpha)\right)$, so our task reduces to describing $\widetilde{\mathbb{N}}_{2}(\alpha)$.

Rotation of a support plane for $\Delta$ about an edge from one face of $\Delta$ to another generates a curve in $\widetilde{\mathbb{N}}_{2}(\alpha)$. This is depicted in Figure $2(\mathrm{a})$, which shows $\Delta$ and the 
rotation of support plane $P$ to support plane $Q$ about the edge $e$. The corresponding curve in $\widetilde{\mathbb{G}}_{1} \cong S^{2}$ is shown in (b) as the arc of the great circle from the outward unit normal $\mathbf{v}_{P}$ to $\mathbf{v}_{Q}$. We can "walk" a support plane around $\Delta$ by first rotating about an edge to a face, then rotating about a different edge of that face to another face, and so on. It follows that $\widetilde{\mathbb{N}}_{2}(\alpha)$ is the union of six curves, one for each edge of $\Delta$. The corresponding set in $\widetilde{\mathbb{G}}_{1} \cong S^{2}$ is the union of the six great circle arcs shown in (b). Note that $\widetilde{\mathbb{N}}_{2}(\alpha)$ is homeomorphic to the 1-skeleton of $\Delta$ (more accurately, to the 1-skeleton of the dual polytope to $\Delta$, but $\Delta$ is self-dual), so that $\mathbb{N}_{2}(\alpha)$ has topological dimension 1 .

Example 2.10. Let $\mathbb{T}^{2}=\mathbb{R}^{2} / \mathbb{Z}^{2}$ denote the 2-dimensional torus. The matrices

$$
A=\left[\begin{array}{ll}
1 & 1 \\
2 & 1
\end{array}\right] \quad \text { and } \quad B=\left[\begin{array}{ll}
2 & 1 \\
3 & 2
\end{array}\right]
$$

in $G L(2, \mathbb{Z})$ induce automorphisms of $\mathbb{T}^{2}$ (which do not commute). The eigenvalues of $A$ are $\lambda_{1}=1+\sqrt{2}$ and $\lambda_{2}=1-\sqrt{2}$, and let $E_{1}$ and $E_{2}$ be the corresponding eigenspaces in $\mathbb{R}^{2}$. Similarly, $B$ has eigenvalues $\mu_{1}=2+\sqrt{3}$ and $\mu_{2}=2-\sqrt{3}$, with eigenspaces $F_{1}$ and $F_{2}$.

Let

$$
C=A \otimes I=\left[\begin{array}{llll}
1 & 1 & 0 & 0 \\
2 & 1 & 0 & 0 \\
0 & 0 & 1 & 1 \\
0 & 0 & 2 & 1
\end{array}\right] \text { and } \quad D=I \otimes B=\left[\begin{array}{llll}
2 & 0 & 1 & 0 \\
0 & 2 & 0 & 1 \\
3 & 0 & 2 & 0 \\
0 & 3 & 0 & 2
\end{array}\right]
$$

Both $C$ and $D$ act on $\mathbb{R}^{2} \otimes \mathbb{R}^{2}=\bigoplus_{i, j=1}^{2} E_{i} \otimes F_{j}$, and they commute. Hence they define an action $\alpha$ of $\mathbb{Z}^{2}$ on $\mathbb{T}^{4}$ given by $\alpha^{(m, n)}=C^{m} D^{n}=A^{m} \otimes B^{n}$. The 1-dimensional spaces $E_{i} \otimes F_{j}$ are common eigenspaces for $C$ and $D$, and $\alpha^{(m, n)}$ on $E_{i} \otimes F_{j}$ is multiplication by $\lambda_{i}^{m} \mu_{j}^{n}$.

We first observe that if $(m, n) \neq \mathbf{0}$, then $\alpha^{(m, n)}$ is hyperbolic, hence expansive. For suppose that $\left|\lambda_{i}^{m} \mu_{j}^{n}\right|=1$, so that $\lambda_{i}^{m} \mu_{j}^{n}= \pm 1$. There is an element $\sigma$ in the Galois group of $\mathbb{Q}(\sqrt{2}, \sqrt{3})$ over $\mathbb{Q}$ with $\sigma(\sqrt{2})=-\sqrt{2}$ and $\sigma(\sqrt{3})=\sqrt{3}$. Hence $\lambda_{i}^{m} \mu_{j}^{n}= \pm 1=\sigma( \pm 1)=\sigma\left(\lambda_{i}\right)^{m} \mu_{j}^{n}$, so that $\left(\lambda_{i} / \sigma\left(\lambda_{i}\right)\right)^{m}= \pm 1$, implying that $m=0$. Similarly, $n=0$. This shows that all lines in $\mathbb{G}_{1}$ with rational slope are expansive for $\alpha$, so that $\mathbb{E}_{1}(\alpha)$ is dense in $\mathbb{G}_{1}$.

We next show that $\alpha$ has exactly two nonexpansive lines, both with irrational slope.

For $i=1,2$ let $L_{i}$ be the line in $\mathbb{R}^{2}$ with slope $-\log \left|\lambda_{i}\right| / \log \left|\mu_{1}\right|$, which is numerically about $\mp 0.66925$. (Using $\mu_{2}$ instead of $\mu_{1}$ would give the same lines, since $\log \left|\mu_{2}\right|=-\log \left|\mu_{1}\right|$.) For fixed $t>0$, consider the strip $L_{i}^{t}$ of width $t$ around $L_{i}$. A lattice point $(m, n)$ occurs in $L_{i}^{t}$ exactly when

$$
|m \log | \lambda_{i}|+n \log | \mu_{1}|| \leq t|\log | \lambda_{i}||=c,
$$

which is equivalent to

$$
e^{-c} \leq\left|\lambda_{i}^{m} \mu_{1}^{n}\right| \leq e^{c}
$$

Thus for all $(m, n) \in L_{i}^{t}$, the linear map $\alpha^{(m, n)}$ never spreads points in $E_{i} \otimes F_{1}$ by a factor more than $e^{t \log \lambda_{1}}$. Hence distinct points in $\mathbb{T}^{4}$ can remain arbitrarily close for all iterates in $L_{i}^{t}$, so that each $L_{i}$ is not expansive.

Suppose now that $L$ is a line distinct from $L_{1}$ and $L_{2}$. Choose a unit vector $\mathbf{e} \in L$, and define $\pi: \mathbb{R}^{2} \rightarrow \mathbb{R}$ by $\pi(\mathbf{x})=\mathbf{x} \cdot \mathbf{e}$. Fix $t>0$. Then $L^{t} \cap \mathbb{Z}^{2}$ is unbounded in 
both directions, but has bounded gaps in the sense that there is an $M>0$ such that for every $\mathbf{p} \in L^{t} \cap \mathbb{Z}^{2}$ there are $\mathbf{q}, \mathbf{r} \in L^{t} \cap \mathbb{Z}^{2}$ for which $\pi(\mathbf{q})<\pi(\mathbf{p})<\pi(\mathbf{r})$, $\|\mathbf{p}-\mathbf{q}\| \leq M$, and $\|\mathbf{p}-\mathbf{r}\| \leq M$. If $L$ is rational this is trivial since $L^{t} \cap \mathbb{Z}^{2}$ contains a rank one subgroup of $\mathbb{Z}^{2}$, while if $L$ is irrational this follows from the uniform distribution of an irrational rotation of the circle. Considering each $\alpha^{\mathbf{n}}$ as a linear operator on $\mathbb{R}^{4}$, put

$$
\theta=\max \left\{\left\|\alpha^{\mathbf{n}}\right\|:\|\mathbf{n}\| \leq M\right\}
$$

and

$$
\delta=\frac{1}{10 \theta^{2}} .
$$

Let $\mathbf{v} \in \mathbb{R}^{4}$ with $\mathbf{v} \neq \mathbf{0}$ and $\|\mathbf{v}\|<\delta$. Then there is at least one eigenspace $E_{i} \otimes F_{j}$ for which the coordinate of $\mathbf{v}$ is nonzero. Since $L^{t} \cap \mathbb{Z}^{2}$ is infinite in both directions and $L \neq L_{1}$ or $L_{2}$, it follows that the projection of $L^{t} \cap \mathbb{Z}^{2}$ to the horizontal axis along either $L_{1}$ or $L_{2}$ is unbounded, proving that

$$
\left\{m \log \left|\lambda_{i}\right|+n \log \left|\mu_{j}\right|=\log \left|\lambda_{i}^{m} \mu_{j}^{n}\right|:(m, n) \in L^{t} \cap \mathbb{Z}^{2}\right\}
$$

contains arbitrarily large numbers. Hence there is a $\mathbf{p} \in L^{t} \cap \mathbb{Z}^{2}$ such that $\left\|\alpha^{\mathbf{p}} \mathbf{v}\right\| \geq$ $\delta \theta$. Choose such a $\mathbf{p}$ with $|\pi(\mathbf{p})|$ minimal. It follows from the definition of $\theta$ that $\|\mathbf{p}\|>M$. Since $L^{t} \cap \mathbb{Z}^{2}$ has bounded gaps, there is a $\mathbf{q} \in L^{t} \cap \mathbb{Z}^{2}$ for which $|\pi(\mathbf{q})|<|\pi(\mathbf{p})|$ and $\|\mathbf{p}-\mathbf{q}\| \leq M$. Hence $\left\|\alpha^{\mathbf{q}} \mathbf{v}\right\|<\delta \theta$ so that

$$
\left\|\alpha^{\mathbf{p}} \mathbf{v}\right\|=\left\|\alpha^{\mathbf{p}-\mathbf{q}}\left(\alpha^{\mathbf{q}} \mathbf{v}\right)\right\| \leq \delta \theta^{2}=\frac{1}{10} .
$$

Let $\rho$ be the metric on $\mathbb{T}^{4}$ induced by the Euclidean norm on $\mathbb{R}^{4}$. In particular, the projection map $\rho: \mathbb{R}^{4} \rightarrow \mathbb{T}^{4}$ is an isometry when restricted to sets in $\mathbb{R}^{4}$ of diameter $<1 / 10$. The argument above then shows that if $x, y \in \mathbb{T}^{4}$ with $\rho(x, y)<\delta$, then there is a $\mathbf{p} \in L^{t} \cap \mathbb{Z}^{2}$ such that $\delta \theta<\rho\left(\alpha^{\mathbf{p}} x, \alpha^{\mathbf{p}} y\right) \leq 1 / 10$, proving that $L$ is expansive.

Thus $\alpha$ is a $\mathbb{Z}^{2}$-action for which $\mathbb{N}_{1}(\alpha)$ consists of exactly two lines, $L_{1}$ and $L_{2}$, that are both irrational.

Example 2.11. Let $X$ be the compact dual group of $\mathbb{Z}[1 / 6]$. Since $\phi(t)=2 t$ and $\psi(t)=3 t$ are commuting automorphisms of $\mathbb{Z}[1 / 6]$, we can define a $\mathbb{Z}^{2}$-action $\alpha$ on $X$ by putting $\alpha^{\mathbf{e}_{1}}=\widehat{\phi}$ and $\alpha^{\mathbf{e}_{2}}=\widehat{\psi}$, where $\widehat{\phi}$ is the automorphism of $X$ dual to $\phi$. The inclusion $\mathbb{Z} \hookrightarrow \mathbb{Z}[1 / 6]$ dualizes to a quotient homomorphism $X \rightarrow \mathbb{T}$ under which $\alpha^{\mathbf{e}_{1}}$ transforms to multiplication by 2 on $\mathbb{T}$ and $\alpha^{\mathbf{e}_{3}}$ to multiplication by 3 . In this sense the action $\alpha$ on $X$ is the natural extension of the $Z_{+}^{2}$-action on $\mathbb{T}$ given by multiplication by 2 and 3 .

Duality shows that the kernel $K$ of the quotient map $X \rightarrow \mathbb{T}$ has dual group

$$
\mathbb{Z}[1 / 6] / \mathbb{Z} \cong(\mathbb{Z}[1 / 2] / \mathbb{Z}) \oplus(\mathbb{Z}[1 / 3] / \mathbb{Z}),
$$

so that $K \cong \mathbb{Z}_{2} \oplus \mathbb{Z}_{3}$, where $\mathbb{Z}_{p}$ denotes the $p$-adic integers. Hence locally the group $X$ splits into a direct product of a real interval, $\mathbb{Z}_{2}$, and $\mathbb{Z}_{3}$. This local picture is explained in detail in $[\mathrm{LW}]$. In particular, each factor of this direct product acts like an invariant "subspace," but with arithmetic expansion or contraction on the $\mathbb{Z}_{p}$ factors replacing the geometric expansion or contraction that occurs, for example, in toral automorphisms.

Let $|\cdot|_{p}$ denote the $p$-adic valuation on $\mathbb{Z}_{p}$, and $|\cdot|_{\infty}$ be the usual absolute value on $\mathbb{R}$. Considerations as in the previous example show that $\mathbf{v}=(a, b) \neq \mathbf{0}$ generates a nonexpansive line $\mathbb{R} \mathbf{v}$ if and only if $a \log |2|_{p}+b \log |3|_{p}=0$ for some $p=2,3$, 
or $\infty$. Since $|2|_{3}=1=|3|_{2},|2|_{2}=1 / 2$ and $|3|_{3}=1 / 3$, the three choices of $p$ lead to the three nonexpansive lines $L_{1}, L_{2}, L_{3}$ making angles with the positive horizontal axis of $0, \pi / 2$, and $\tan ^{-1}(-\log 2 / \log 3)$, respectively. Hence $\mathbb{N}_{1}(\alpha)=\left\{L_{1}, L_{2}, L_{3}\right\}$ contains two rational lines and one irrational line.

Example 2.12. Let $\alpha$ be a $\mathbb{Z}^{d}$-action on $X$, and suppose that $A: \mathbb{Z}^{e} \rightarrow \mathbb{Z}^{d}$ is a homomorphism. We define an action $\alpha^{A}$ of $\mathbb{Z}^{e}$ on $X$ by the formula $\left(\alpha^{A}\right)^{\mathbf{n}}=\alpha^{A \mathbf{n}}$. We say that $\alpha^{A}$ is obtained from $\alpha$ by a map of parameters.

Some useful special cases of this are

(1) $e=d$ and $A \in G L(d, \mathbb{Z})$,

(2) $e<d$ and $A$ embeds $\mathbb{Z}^{e}$ as a sublattice of $\mathbb{Z}^{d}$, and

(3) $e>d$ and $A$ is surjective, so that the action is "lifted" to a higher dimensional action.

It is easy to verify that a subspace $V$ of $R^{e}$ is expansive for $\alpha^{A}$ if and only if $A(V)$ is expansive for $\alpha$.

\section{Nonexpansive subspaces}

This section develops coding techniques, and applies them to prove that each $\mathbb{Z}^{d}$-action $\alpha$ has nonexpansive subspaces of all dimensions $\leq d-1$. More precisely, we will show that if $V$ is nonexpansive for $\alpha$ and $\operatorname{dim} V \leq d-2$, then $V$ is contained in a $(d-1)$-dimensional nonexpansive subspace. Consequently, $\mathbb{N}_{k}(\alpha)$ is the set of all $k$-dimensional subspaces contained in at least one of the subspaces in $\mathbb{N}_{d-1}(\alpha)$, so that $\mathbb{N}_{d-1}(\alpha)$ determines the other $\mathbb{N}_{k}(\alpha)$.

If a $\mathbb{Z}^{d}$-action $\alpha$ is not expansive, then all subspaces of $\mathbb{R}^{d}$ are nonexpansive, and our results are trivially true. Therefore we will assume throughout this section that $\alpha$ is a fixed expansive $\mathbb{Z}^{d}$-action on $(X, \rho)$, and also that $\delta$ is a fixed expansive constant for $\alpha$.

Definition 3.1. Let $E$ and $F$ be subsets of $\mathbb{R}^{d}$. Say that $E$ codes $F$ provided that, for every $\mathbf{v} \in \mathbb{R}^{d}$, if $\rho_{\alpha}^{E+\mathbf{v}}(x, y) \leq \delta$ then $\rho_{\alpha}^{F+\mathbf{v}}(x, y) \leq \delta$.

For example, a subspace $V$ of $\mathbb{R}^{d}$ is expansive if and only if $V^{t}$ codes $\mathbb{R}^{d}$ for all large $t$. Observe that the coding relation is transitive: if $E$ codes $F$ and $F$ codes $G$, then $E$ codes $G$. The definition of coding builds in translation invariance, so that if $E$ codes $F$, then $E+\mathbf{v}$ codes $F+\mathbf{v}$ for every $\mathbf{v} \in \mathbb{R}^{d}$.

For a subspace $V$ of $\mathbb{R}^{d}$, let $\pi_{V}$ denote orthogonal projection to $V$ along its orthogonal complement $V^{\perp}$, so that $\pi_{V}+\pi_{V^{\perp}}=I$. Then clearly $V^{t}=\left\{\mathbf{v} \in \mathbb{R}^{d}\right.$ : $\left.\left\|\pi_{V^{\perp}}(\mathbf{v})\right\| \leq t\right\}$. We define

$$
V^{t}(r)=\left\{\mathbf{v} \in \mathbb{R}^{d}:\left\|\pi_{V}(\mathbf{v})\right\| \leq r \text { and }\left\|\pi_{V^{\perp}}(\mathbf{v})\right\| \leq t\right\} .
$$

For example, if $d=3$ and $\operatorname{dim} V=2$, then $V^{t}(r)$ is a disc of radius $r$ and thickness $2 t$. Observe that

$$
V^{s}(q)+V^{t}(r)=V^{s+t}(q+r)
$$

for all $s, t, q, r \geq 0$.

The following gives a "finite" version of expansiveness which is an analogue of the sliding block codes of symbolic dynamics.

Lemma 3.2. Let $V$ be an expansive subspace for $\alpha$. There is a $t>0$ with the property that for every $s>0$ there is an $r>0$ such that $V^{t}(r)$ codes $V^{s}(0)$. Hence $V^{t}(r+a)$ codes $V^{s}(a)$ for all $a>0$. 


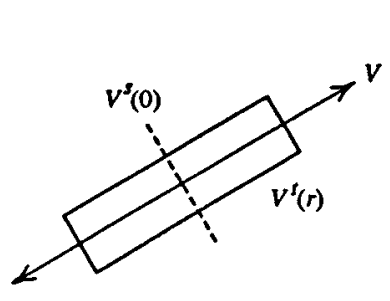

(a)

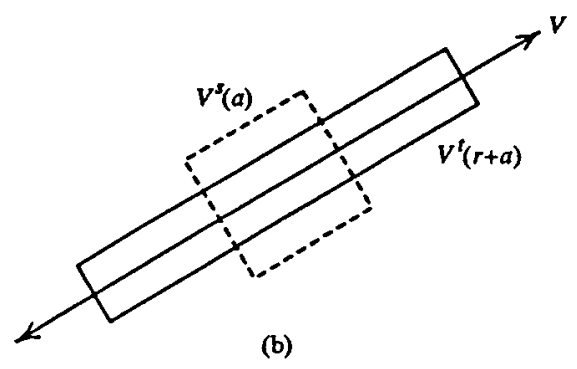

(b)

Figure 3. $V^{t}(r)$ codes $V^{s}(0)$, so $V^{t}(r+a)$ codes $V^{s}(a)$.

Proof. By Lemma 2.3, $V$ has an expansive radius $u>0$, so that $\rho_{\alpha}^{V^{u}}(x, y) \leq \delta$ implies that $x=y$. Put $t=u+d$.

To show that $t$ has the required property, fix an $s>0$. Since $\alpha$ acts by continuous maps, there is a $\gamma>0$ such that if $\rho(x, y) \leq \gamma$, then $\rho_{\alpha}^{B(s+d)}(x, y) \leq \delta$. A compactness argument shows that there is an $r>d$ such that $\rho_{\alpha}^{V^{u}(r-d)}(x, y) \leq \delta$ implies that $\rho(x, y) \leq \gamma$.

We will use these choices of $t$ and $r$ to show that $V^{t}(r) \operatorname{codes} V^{s}(0)$. Let $\mathbf{v} \in \mathbb{R}^{d}$, and suppose that $\rho_{\alpha}^{V^{t}(r)+\mathbf{v}}(x, y) \leq \delta$. Choose $\mathbf{n} \in \mathbb{Z}^{d}$ with $\|\mathbf{v}-\mathbf{n}\|<d$. Then $V^{t}(r)+\mathbf{v} \supset V^{u}(r-d)+\mathbf{n}$, so that $\rho_{\alpha}^{V^{u}(r-d)}\left(\alpha^{\mathbf{n}} x, \alpha^{\mathbf{n}} y\right) \leq \delta$. Hence $\rho\left(\alpha^{\mathbf{n}} x, \alpha^{\mathbf{n}} y\right) \leq \gamma$, so $\rho_{\alpha}^{B(s+d)+\mathbf{n}}(x, y) \leq \delta$. But $V^{s}(0)+\mathbf{v} \subset B(s+d)+\mathbf{n}$, proving that $\rho_{\alpha}^{V^{s}(0)+\mathbf{v}}(x, y) \leq$ $\delta$, and that $V^{t}(r)$ codes $V^{s}(0)$.

Next, observe that if $G \subset \mathbb{R}^{d}$ and if $E$ codes $F$, then $E+G$ codes $F+G$. Apply this observation with $E=V^{t}(r), F=V^{s}(0)$, and $G=V^{0}(a)$, and use (3-1), to obtain the final statement (see Figure 3, where in each drawing the solid rectangle codes the dotted region).

The preceding lemma has a converse, which captures the idea behind Examples 2.8 and 2.9 .

Lemma 3.3. Suppose that $V$ is a subspace, and that $r$, $t$, and $\epsilon$ are positive numbers for which $V^{t}(r)$ codes $V^{t+\epsilon}(0)$. Then $V$ is expansive.

Proof. If $V^{t}(r)$ codes $V^{t+\epsilon}(0)$, then use of (3-1) shows that $V^{t}(r+a)$ codes $V^{t+\epsilon}(a)$ for all $a>0$. Hence $V^{t}$ codes $V^{t+\epsilon}$, and so codes $V^{t+2 \epsilon}$, and so on. This shows that $V^{t}$ codes $\mathbb{R}^{d}$, i.e., that $V$ is expansive.

The next result shows that expansiveness is an open condition.

Lemma 3.4. Let $V$ be an expansive $k$-plane for $\alpha$. Then there are $r=r_{V}, t=t_{V}$, and a neighborhood $\mathcal{N}_{V}$ of $V$ in $\mathbb{G}_{k}$ such that, for every $W \in \mathcal{N}_{V}$, we have that $W^{t}(r)$ codes $W^{t+1}(0)$. Hence each $W \in \mathcal{N}_{V}$ is expansive, and so $\mathbb{E}_{k}(\alpha)$ is open in $\mathbb{G}_{k}$.

Proof. By Lemma 3.2, there are $s$ and $u$ so that $V^{u}(s)$ codes $V^{u+3}(1)$. Put $r=s+1$ and $t=u+1$. For $W$ sufficiently close to $V, W^{t}(r)=W^{u+1}(s+1)$ contains $V^{u}(s)$, which codes $V^{u+3}(1)$, which contains $W^{u+2}(0)=W^{t+1}(0)$. Figure 4 illustrates these relations. Lemma 3.3 now shows that each $W$ sufficiently close to $V$ is expansive, hence that $\mathbb{E}_{k}(\alpha)$ is open. 


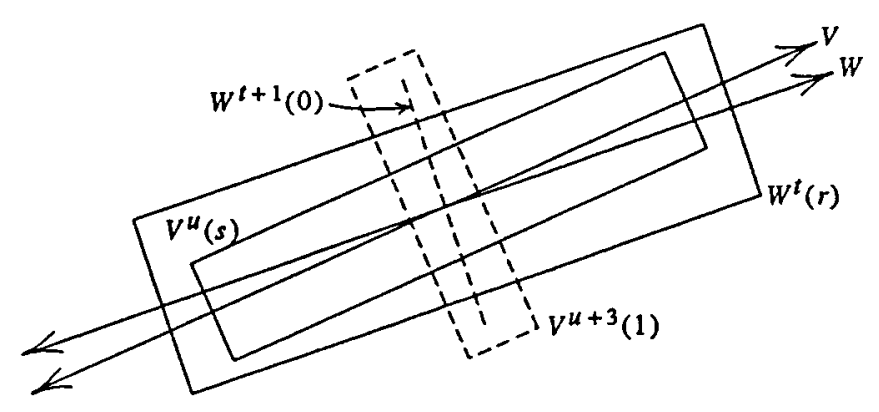

FiguRE 4. Coding relations which show that expansiveness is an open condition.

The next result shows that the coding in Lemma 3.4 can be made uniform on compact subsets of expansive subspaces.

Lemma 3.5. Let $\mathcal{K}$ be a compact subset of $\mathbb{E}_{k}(\alpha)$. Then there are $r>0$ and $t>0$ such that $W^{t}(r)$ codes $W^{t+1}(0)$ for every $W \in \mathcal{K}$.

Proof. By Lemma 3.4, for each $V \in \mathcal{K}$ there are $r_{V}>0, t_{V}>0$, and a neighborhood $\mathcal{N}_{V}$ of $V$ in $\mathbb{G}_{k}$ such that $W^{t_{V}}\left(r_{V}\right)$ codes $W^{t_{V}+1}(0)$ for all $W \in \mathcal{N}_{V}$. Since $\mathcal{K}$ is compact, it is covered by some finite collection $\mathcal{N}_{V_{1}}, \ldots, \mathcal{N}_{V_{m}}$. Observe that if $W^{u}(s)$ codes $W^{u+1}(0)$, then $W^{U}(S)$ codes $W^{U+1}(0)$ for all $U \geq u$ and $S \geq s$. Therefore $r=\max _{1 \leq j \leq m}\left\{r_{V_{j}}\right\}$ and $t=\max _{1 \leq j \leq m}\left\{t_{V_{j}}\right\}$ satisfy the conclusion.

We are now ready for the main result of this section.

Theorem 3.6. Suppose that $V$ is a nonexpansive subspace for $\alpha$ with dimension $\leq d-2$. Then $V$ is contained in a $(d-1)$-dimensional nonexpansive subspace.

Proof. Fix $V \in \mathbb{N}_{k}(\alpha)$, where $k \leq d-2$. Let $\mathcal{K}=\left\{W \in \mathbb{G}_{d-1}: W \supset V\right\}$, which is a compact submanifold of $\mathbb{G}_{d-1}$. Suppose that every subspace in $\mathcal{K}$ is expansive. Then by Lemma 3.5, there are $r, t>0$ such that $W^{t}(r)$ codes $W^{t+1}(0)$ for all $W \in \mathcal{K}$. It follows that there is an $R_{0}>0$ so that for all $R \geq R_{0}$ the boundary of $V^{R}$ is sufficiently "flat" so that the following holds. For all $R \geq R_{0}$ and all $\mathbf{v} \in V^{R+1 / 2}$, there is a $W \in \mathcal{K}$ and $\mathbf{u} \in \mathbb{R}^{d}$ such that $W^{t}(r)+\mathbf{u} \subset V^{R}$ and $\mathbf{v} \in W^{t+1}(0)+\mathbf{u}$. Figure 5 depicts this situation when $d=2$ and $V=\{0\}$, corresponding to the assumption that every line is expansive. Hence $V^{R}$ codes $V^{R+1 / 2}$ for all $R \geq R_{0}$. But repeated application of this implies that $V^{R_{0}}$ codes all of $\mathbb{R}^{d}$, contradicting nonexpansiveness of $V$. Hence $\mathcal{K}$ must contain at least one nonexpansive subspace.

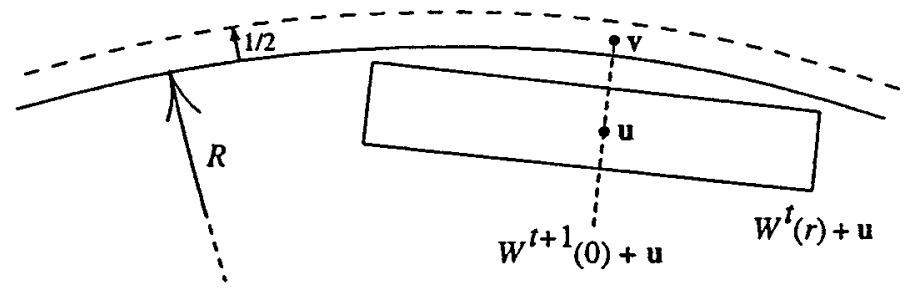

Figure 5. A large ball codes a bigger ball. 


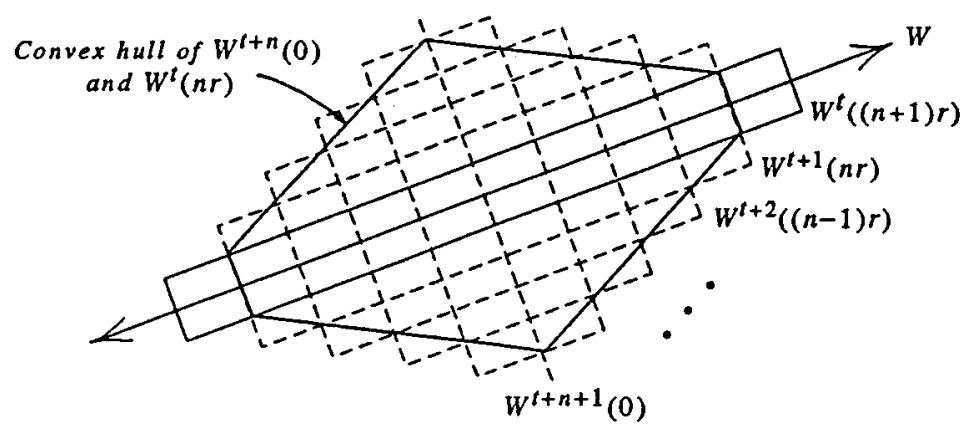

Figure 6. A strip codes a convex hull.

This result leads to our description of the expansive subdynamics of a general $\mathbb{Z}^{d}$-action.

Theorem 3.7. Let $\alpha$ be an arbitrary $\mathbb{Z}^{d}$-action on an infinite compact metric space. For each $k$ with $0 \leq k \leq d-1$, the set $\mathbb{N}_{k}(\alpha)$ of nonexpansive subspaces of dimension $k$ is a nonempty compact set in $\mathbb{G}_{k}$. A subspace is nonexpansive if and only if it is contained in some subspace in $\mathbb{N}_{d-1}(\alpha)$, so that $\mathbb{N}_{d-1}(\alpha)$ determines all the other $\mathbb{N}_{k}(\alpha)$.

Proof. Since $X$ is infinite, the zero subspace $\{0\}$ is nonexpansive. By Theorem 3.6, this subspace is contained in a nonexpansive $(d-1)$-dimensional subspace, all of whose subspaces are also nonexpansive (see Remark 2.6). Thus all the $\mathbb{N}_{k}(\alpha)$ are nonempty for $0 \leq k \leq d-1$, and they are compact by Proposition 3.4. The second part follows directly from Theorem 3.6 and Remark 2.6.

We record now an easy consequence of Lemma 3.5 which we will need in $\S 6$ and in $\S 8$.

Proposition 3.8. Let $\mathcal{K}$ be a compact subset of $\mathbb{E}_{k}(\alpha)$. Then there are $r>0$ and $t>0$ such that, for every $W \in \mathcal{K}$ and $n \in \mathbb{N}, W^{t}((n+1) r)$ codes the convex hull of $W^{t+n}(0)$ and $W^{t}(n r)$.

Proof. Choose $r$ and $t$ as in Lemma 3.5. We may clearly assume that $t \geq 1$. Suppose $W \in \mathcal{K}$. Then $W^{t}((n+1) r)$ codes $W^{t+1}(n r)$, which codes $W^{t}((n-1) r)$, and so on. Thus $W^{t}(n r)$ codes $W^{t+j}((n-j) r)$ for $0 \leq j \leq n$. Hence the union of these sets contains the required convex hull (see Figure 6).

In his Ph.D. thesis, Sol Schwartzman proved that there are no "one-sided expansive" homeomorphisms, except on finite spaces.

Theorem 3.9 (Schwartzman). If $T$ is a homeomorphism of an infinite compact metric space $(X, \rho)$ and $\delta>0$, then there are distinct points $x, y$ in $X$ such that $\rho\left(T^{n} x, T^{n} y\right) \leq \delta$ for all $n \geq 0$.

Schwartzman never published this result, but it is reported in [GH, 10.30]. King gives a direct proof [Ki, Thm. 2.1] (attributed to Boyle, Geller and Propp), and also shows that $x$ and $y$ can be found in different orbits [Ki, Thm. 2.6]. Schwartzman's result is also a quick corollary of [AKM, p. 316]. This result was a key ingredient in our original, more intricate, proof of Theorem 3.6. It has also guided us in 
certain constructions (see Theorem 6.33) as well as providing obstructions in other situations.

One can prove Schwartzman's result for an expansive homeomorphism in two steps: first, show that if $[0, \infty)$ codes $(-\infty, 0]$, then for some $N$ the bounded interval $[0, N]$ codes $(-\infty, 0]$; second, check that this implies the space is finite (we thank Jim Propp and Will Geller for helping us understand this). The first step generalizes to higher dimensional actions as follows. The proof is a compactness argument similar to previous ones, and is omitted.

Proposition 3.10. Let $W$ be an expansive $k$-plane for $\alpha$, and $V$ be $a(k-1)$-plane contained in $W$. Denote by $W_{+}$and $W_{-}$the two closed half-spaces of $W$ with boundary $V$. If $W_{+}$codes $W_{-}$, then there is a bounded neighborhood of $V$ that also codes $W_{-}$.

Remark 3.11. The last proposition, Example 2.9, and the "causal cones" of Milnor [Mi] suggest an oriented notion of expansiveness for codimension 1 subspaces. This will be useful in describing the algebraic examples of $\S 7$.

Let $\widetilde{\mathbb{G}}_{d-1}$ denote the compact space of all oriented $(d-1)$-dimensional subspaces of $\mathbb{R}^{d}$. The map $p_{d-1}: \widetilde{\mathbb{G}}_{d-1} \rightarrow \mathbb{G}_{d-1}$ which forgets orientation is a 2-to-1 covering map (we used $p_{2}$ in Example 2.9). If $V \in \mathbb{G}_{d-1}$ and $p_{d-1}^{-1}(V)=\left\{V^{+}, V^{-}\right\}$, then each $V^{ \pm}$determines a half-space $H_{V}^{ \pm}$with boundary $V$. Say that $V^{ \pm}$is a causal plane (with respect to an action $\alpha$ ) if some bounded thickening of $V$ codes $H_{V}^{ \pm}$. Equivalently, by compactness, $V^{ \pm}$is a causal plane if and only if $H_{V}^{\mp}$ is an expansive set for $\alpha$. Clearly a $(d-1)$-plane $V$ is expansive if and only if both $V^{+}$and $V^{-}$are causal planes.

Denote by $\mathcal{C}_{\alpha}$ the set of all causal planes for $\alpha$. Then $\mathcal{C}_{\alpha}$ is an open subset of $\widetilde{\mathbb{G}}_{d-1}$ for reasons similar to the proof of Lemma 3.4. Let

$$
D: \mathbb{G}_{d-1} \rightarrow \widetilde{\mathbb{G}}_{d-1} \times \widetilde{\mathbb{G}}_{d-1}
$$

be defined by $D(V)=\left(V^{+}, V^{-}\right)$. The last remark in the previous paragraph shows that

Consequently

$$
\mathbb{E}_{d-1}(\alpha)=D^{-1}\left(\mathcal{C}_{\alpha} \times \mathcal{C}_{\alpha}\right)
$$

$$
\mathbb{N}_{d-1}(\alpha)=p_{d-1}\left(\widetilde{\mathbb{G}}_{d-1} \backslash \mathcal{C}_{\alpha}\right),
$$

which we used in Example 2.9.

\section{Realization}

Let $\alpha$ be a $\mathbb{Z}^{d}$-action. Theorem 3.7 shows that the structure of the nonexpansive sets $\mathbb{N}_{k}(\alpha)$ for $1 \leq k \leq d-1$ is completely determined by $\mathbb{N}_{d-1}(\alpha)$. Which compact sets in $\mathbb{G}_{d-1}$ can occur as $\mathbb{N}_{d-1}(\alpha)$ for some $\mathbb{Z}^{d}$-action $\alpha$ ?

When $d=2$ we can give a nearly complete answer: if $\mathcal{L} \subset \mathbb{G}_{1}$ is a compact set of lines that is not a singleton set containing one irrational line, then we will construct a $\mathbb{Z}^{2}$-action $\alpha$ for which $\mathbb{N}_{1}(\alpha)=\mathcal{L}$ (Theorem 4.3). For $d>2$, our results are less complete (see Problem 9.1). Our basic method is contained in the following construction.

Proposition 4.1. Let $d=2$, and suppose that $\mathcal{L}$ is a compact set of lines in $\mathbb{G}_{1}$ such that $|\mathcal{L}| \geq 2$ and $\mathcal{L}$ contains an isolated line. Then there is a $\mathbb{Z}^{2}$-action $\alpha$ for which $\mathbb{N}_{1}(\alpha)=\mathcal{L}$. 
Proof. Recall from Example 2.7 that the $\mathbb{Z}^{2}$-shift on $\{0,1\} \mathbb{Z}^{2}$ is expansive. We will construct a shift-invariant compact subset $X \subset\{0,1\}^{\mathbb{Z}^{2}}$, put $\alpha$ to be the restriction of the $\mathbb{Z}^{2}$-shift to $X$, and show that $\mathbb{N}_{1}(\alpha)=\mathcal{L}$.

Let $K$ be a fixed, isolated line in $\mathcal{L}$ (we use the topology induced from $\mathbb{G}_{1}$ ). Then there is a connected neighborhood $\mathcal{N}$ of $K$ in $\mathbb{G}_{1}$ for which $\mathcal{N} \cap \mathcal{L}=\{K\}$.

For any line $L \in \mathbb{G}_{1}$, define an L-strip in $\mathbb{Z}^{2}$ to be any subset $S \subset \mathbb{Z}^{2}$ such that there is a vector $\mathbf{v} \in \mathbb{R}^{2}$ for which

$$
\left\{\mathbf{n} \in \mathbb{Z}^{2}: \operatorname{dist}(\mathbf{n}, L+\mathbf{v})<2\right\} \subset S \subset\left\{\mathbf{n} \in \mathbb{Z}^{2}: \operatorname{dist}(\mathbf{n}, L+\mathbf{v}) \leq 2\right\} .
$$

Thus an $L$-strip is the set of lattice points lying at distance $<2$ from a translate of $L$, plus possibly some lattice points at distance exactly 2 .

Since $K$ is isolated in $\mathcal{L}$, there are $\mathbf{p}, \mathbf{q} \in \mathbb{Z}^{2}$ such that

1. The lines generated by $\mathbf{p}$ and by $\mathbf{q}$ are both in $\mathcal{N}$,

2. $K$ intersects the positive cone generated by $\mathbf{p}$ and $\mathbf{q}$, and

3. for every $L \in \mathcal{L} \backslash\{K\}$ and every $L$-strip $S$,

$$
\operatorname{dist}(S, S+\mathbf{q}) \geq 10 \operatorname{dist}(S, S+\mathbf{p}) \geq 100 .
$$

Thus for each $L \in \mathcal{L} \backslash\{K\}, L$-strips are separated by at least 10 when translated by $\mathbf{p}$, and by at least 100 when translated by $q$.

Let $\pi_{K^{\perp}}$ denote orthogonal projection in $\mathbb{R}^{2}$ to $K^{\perp}$ along $K$. Define a translation sequence to be a bi-infinite sequence $\mathcal{T}=\left\{\ldots, \mathbf{k}_{-1}, \mathbf{k}_{0}, \mathbf{k}_{1}, \ldots\right\}$ of lattice points $\mathbf{k}_{j} \in \mathbb{Z}^{2}$ such that

(i) $\mathbf{k}_{j+1}-\mathbf{k}_{j}=\mathbf{p}$ or $\mathbf{q}$,

(ii) $\operatorname{diam}\left\{\pi_{K^{\perp}}\left(\mathbf{k}_{j}-\mathbf{k}_{i}\right): i, j \in \mathbb{Z}\right\} \leq 2 \max \left\{\left\|\pi_{K^{\perp}}(\mathbf{p})\right\|,\left\|\pi_{K^{\perp}}(\mathbf{q})\right\|\right\}$.

Observe that if $\mathcal{T}=\left\{\mathbf{k}_{j}\right\}$ is a translation sequence, then so is $\mathcal{T}+\mathbf{n}=\left\{\mathbf{k}_{j}+\mathbf{n}\right\}$ for every $\mathbf{n} \in \mathbb{Z}^{2}$.

We now define $X \subset\{0,1\}^{\mathbb{Z}^{2}}$. For every line $L \in \mathcal{L} \backslash\{K\}$, every $L$-strip $S$, and every translation sequence $\mathcal{T}=\left\{\mathbf{k}_{j}\right\}$, define a point $x_{S, \mathcal{T}}$ in $\{0,1\}^{\mathbb{Z}^{2}}$ by

$$
x_{S, \mathcal{T}}(\mathbf{m})= \begin{cases}1 & \text { if } \mathbf{m} \in \bigcup_{j \in \mathbb{Z}}\left(S+\mathbf{k}_{j}\right), \\ 0 & \text { otherwise. }\end{cases}
$$

Note that the translates $S+\mathbf{k}_{j}$ are mutually separated by at least 10 , and that the separations $\operatorname{dist}\left(S+\mathbf{k}_{j}, S+\mathbf{k}_{j+1}\right)$ assume just two values, a small one when $\mathbf{k}_{j+1}-\mathbf{k}_{j}=\mathbf{p}$ and a large one when the difference is $\mathbf{q}$. Let $X$ denote the set of all such $x_{S, \mathcal{T}}$. We will show that $X$ is compact, $\mathbb{Z}^{2}$-shift invariant, and that $\mathbb{N}_{1}(\alpha)=\mathcal{L}$.

Since the property of being an $L$-strip or of being a translation sequence is preserved under translating by a lattice point, $X$ is $\mathbb{Z}^{2}$-shift invariant.

Next, suppose that a sequence $\left\{x_{S_{n}, \mathcal{T}_{n}}\right\}$ in $X$ converges to a point $x \in\{0,1\}^{\mathbb{Z}^{2}}$. Each $S_{n}$ is an $L_{n}$-strip for some $L_{n}$, and the lines $L_{n}$ must clearly converge to a line $L$, which is also in $\mathcal{L} \backslash\{K\}$ since $\mathcal{L} \backslash\{K\}$ is compact. By translating if necessary, we may assume that each $\mathcal{T}_{n}=\left\{\mathbf{k}_{j, n}\right\}$ has 0 th term $\mathbf{k}_{0, n}=\mathbf{0}$. Since the separations ("small" or "large") between adjacent strips determine the corresponding differences in translation vectors, it follows that for every $J>0$ there is an $n_{J}$ such that $\mathbf{k}_{j+1, n}-\mathbf{k}_{j, n}$ are all equal for $n \geq n_{J}$ and $|j| \leq J$. Our normalization $\mathbf{k}_{0, n}=\mathbf{0}$ then shows that $\lim _{n \rightarrow \infty} \mathbf{k}_{j, n}$ exists. If $\mathbf{k}_{j}$ denotes its value, then $\mathcal{T}=\left\{\mathbf{k}_{j}\right\}$ is clearly also a translation sequence. The normalization $\mathbf{k}_{0, n}=\mathbf{0}$ also shows that the $L_{n}$-strips 
$S_{n}$ converge to an $L$-strip $S$, in the sense that for every $r>0$, if $B(r)$ denotes the ball of radius $r$ in $\mathbb{R}^{2}$, then $S_{n} \cap B(r)$ eventually equals $S \cap B(r)$. Hence

$$
\bigcup_{j \in \mathbb{Z}}\left(S_{n}+\mathbf{k}_{j, n}\right) \quad \text { converges to } \bigcup_{j \in \mathbb{Z}}\left(S+\mathbf{k}_{j}\right)
$$

in the same sense. Thus $x_{S_{n}, \mathcal{I}_{n}} \rightarrow x_{S, \mathcal{T}}$ in $\{0,1\}^{\mathbb{Z}^{2}}$, so that $x=x_{S, \mathcal{T}}$. This shows that $X$ is compact.

Finally, we show that $\mathbb{N}_{1}(\alpha)=\mathcal{L}$. First, let $L \in \mathcal{L} \backslash\{K\}$ and suppose that $u>0$. The condition (ii) allows sufficient freedom that we can easily find distinct translation sequences $\mathcal{T}=\left\{\mathbf{k}_{j}\right\}$ and $\mathcal{T}^{\prime}=\left\{\mathbf{k}_{j}^{\prime}\right\}$, normalized so that $\mathbf{k}_{0}=\mathbf{k}_{0}^{\prime}=\mathbf{0}$, whose intersections with $B(u+2)$ agree. If $S$ is any $L$-strip, and we put $x=x_{S, \mathcal{T}}$ and $y=x_{S, \mathcal{T}^{\prime}}$, then $x$ and $y$ agree on $L^{u}$, but $x \neq y$. Thus $L \in \mathbb{N}_{1}(\alpha)$, so that $\mathcal{L} \backslash\{K\} \subset \mathbb{N}_{1}(\alpha)$. Also, $K$ is nonexpansive, but for a different reason. Let $u>0$, and fix a translation sequence $\mathcal{T}$. Then there is an $\mathbf{n} \in \mathbb{Z}^{2}$ such that $K^{u} \cap(\mathcal{T}+\mathbf{n})=\varnothing$. Let $L \in \mathcal{L} \backslash\{K\}$, and let $S, S^{\prime}$ be $L$-strips whose symmetric difference is $\{\mathbf{n}\}$. Then $x_{S, \mathcal{T}}$ and $x_{S^{\prime}, \mathcal{T}}$ are distinct, but agree on $K^{u}$. This proves that $K \in \mathbb{N}_{1}(\alpha)$. Hence $\mathcal{L} \subset \mathbb{N}_{1}(\alpha)$.

To establish the reverse inclusion, let $M \in \mathbb{G}_{1} \backslash \mathcal{L}$. There is a $u>0$ such that, for every $x=x_{S, \mathcal{T}} \in X$, the configuration $\left\{x(\mathbf{n}): \mathbf{n} \in M^{u}\right\}$ determines the separations of adjacent strips. Hence, if we normalize so that $\mathcal{T}=\left\{\mathbf{k}_{j}\right\}$ has $\mathbf{k}_{0}=\mathbf{0}$, then $\left\{x(\mathbf{n}): \mathbf{n} \in M^{u}\right\}$ determines $\mathcal{T}$. Let $S$ be an $L$-strip, where $L \in \mathcal{L} \backslash\{K\}$. We can assume that $u$ is large enough so that

$$
S \subset \bigcup_{j \in \mathbb{Z}}\left\{\left(S+\mathbf{k}_{j}\right) \cap M^{u}-\mathbf{k}_{j}\right\},
$$

i.e., $\left\{x(\mathbf{n}): \mathbf{n} \in M^{u}\right\}$ determines $x$. This shows that $M$ is expansive, completing the proof.

If $\alpha$ and $\beta$ are $\mathbb{Z}^{d}$-actions on $\left(X, \rho_{X}\right)$ and $\left(Y, \rho_{Y}\right)$, respectively, define their product action $\alpha \times \beta$ on $X \times Y$ by $(\alpha \times \beta)^{\mathbf{n}}=\alpha^{\mathbf{n}} \times \beta^{\mathbf{n}}$. We use the metric

$$
\rho_{X \times Y}\left((x, y),\left(x^{\prime}, y^{\prime}\right)\right)=\max \left\{\rho_{X}\left(x, x^{\prime}\right), \rho_{Y}\left(y, y^{\prime}\right)\right\} .
$$

The nonexpansive set is "additive" over products.

Lemma 4.2. Let $\alpha$ and $\beta$ be $\mathbb{Z}^{d}$-actions. For $1 \leq k \leq d$, we have that

$$
\mathbb{N}_{k}(\alpha \times \beta)=\mathbb{N}_{k}(\alpha) \cup \mathbb{N}_{k}(\beta) .
$$

Proof. Let $V \in \mathbb{N}_{k}(\alpha)$. Then for every $\delta>0$ and $t>0$, there are $x \neq x^{\prime}$ in $X$ such that $\rho_{\alpha}^{V^{t}}\left(x, x^{\prime}\right) \leq \delta$. For each $y \in Y$ we therefore have

$$
\rho_{\alpha \times \beta}^{V^{t}}\left((x, y),\left(x^{\prime}, y\right)\right) \leq \delta,
$$

proving that $V \in \mathbb{N}_{k}(\alpha \times \beta)$. Thus $\mathbb{N}_{k}(\alpha) \subset \mathbb{N}_{k}(\alpha \times \beta)$, and similarly $\mathbb{N}_{k}(\beta) \subset$ $\mathbb{N}_{k}(\alpha \times \beta)$.

The reverse inclusion $\mathbb{N}_{k}(\alpha \times \beta) \subset \mathbb{N}_{k}(\alpha) \cup \mathbb{N}_{k}(\beta)$ follows from the definition of $\rho_{X \times Y}$.

Theorem 4.3. Let $d=2$, and $\mathcal{L}$ be a compact set in $\mathbb{G}_{1}$ that is not a singleton containing just one irrational line. Then there is a $\mathbb{Z}^{2}$-action $\alpha$ with $\mathbb{N}_{1}(\alpha)=\mathcal{L}$. 
Proof. First suppose that $\mathcal{L}=\{L\}$, with $L$ rational. Let $T$ be an expansive homeomorphism of a space $X$, and define the $\mathbb{Z}^{2}$-action $\beta$ on $X$ by $\beta^{(m, n)}=T^{n}$. Clearly $\mathbb{N}_{1}(\beta)$ is just the horizontal axis, say $\{K\}$. Then there is an $A \in G L(2, \mathbb{Z})$ for which $A(K)=L$. Use the map of parameters $\alpha=\beta^{A}$ (see Example 2.12) to obtain $\mathbb{N}_{1}(\alpha)=\{L\}$.

Next, suppose that $|\mathcal{L}| \geq 2$. Pick $K \neq L$ in $\mathcal{L}$. There are disjoint open neighborhoods $\mathcal{M}$ of $K$ and $\mathcal{N}$ of $L$ in $\mathbb{G}_{1}$. Then $\mathcal{L}_{1}=\{K\} \cup(\mathcal{L} \backslash \mathcal{M})$ and $\mathcal{L}_{2}=\{L\} \cup(\mathcal{L} \backslash \mathcal{N})$ are compact, contain at least two elements each, and $\mathcal{L}=\mathcal{L}_{1} \cup \mathcal{L}_{2}$. Then $\mathcal{L}_{1}$ and $\mathcal{L}_{2}$ each satisfy the hypotheses of Proposition 4.1, so there are $\mathbb{Z}^{2}$-actions $\alpha_{1}$ and $\alpha_{2}$ with $\mathbb{N}_{1}\left(\alpha_{1}\right)=\mathcal{L}_{1}$ and $\mathbb{N}_{1}\left(\alpha_{2}\right)=\mathcal{L}_{2}$. By Lemma $4.2, \mathbb{N}_{1}\left(\alpha_{1} \times \alpha_{2}\right)=\mathcal{L}_{1} \cup \mathcal{L}_{2}=\mathcal{L}$.

Recall that $\mathbb{G}_{d-1}$ is homeomorphic to $\mathbb{G}_{1}$ by mapping a $(d-1)$-plane to its normal line. We can therefore think of subsets of $\mathbb{G}_{d-1}$ as being subsets of the sphere $S^{d-1}$ that are invariant under the antipodal map. With this understanding, we define a great sphere in $\mathbb{G}_{d-1}$ to be the intersection of $S^{d-1}$ with a $(d-1)$-dimensional subspace of $\mathbb{R}^{d}$.

Proposition 4.4. Suppose $\mathcal{K}$ is a compact subset of $\mathbb{G}_{d-1}$ which properly contains an isolated great sphere. Then there is a $\mathbb{Z}^{d}$-action $\alpha$ with $\mathbb{N}_{d-1}(\alpha)=\mathcal{K}$.

Proof. This is a straightforward adaptation of the proof of Proposition 4.1. Let $Q$ be the isolated great sphere, and let $K$ be the line perpendicular to the subspace spanned by $Q$. Thus the set of $(d-1)$-planes represented by $Q$ is precisely the set of those $(d-1)$-planes which contain $K$. For each $(d-1)$-plane $V$ in the nonempty closed set $\mathcal{K} \backslash Q$, we define a $V$-strip just as before, after replacing $\mathbb{Z}^{2}$ with $\mathbb{Z}^{d}$. Similarly, use $K$ to define allowed sequences of translation vectors. This yields an action whose nonexpansive $(d-1)$-planes are precisely those in $\mathcal{K} \backslash Q$ together with those containing $K$. We leave the straightforward details to the reader.

Remark 4.5. By Lemma 4.2, we can realize finite unions of the sets $\mathcal{K}$ described in Proposition 4.4. One can also strengthen (and complicate) Lemma 4.4 by considering how the construction adapts given rationality constraints on $(d-1)$-planes in $\mathcal{K}$. For example, we can apply a map of parameters (Example 2.12) to the nonexpansive sets constructed in Theorem 4.3 and Proposition 4.4 to produce further nonexpansive sets.

Remark 4.6. Note that for $d>2$, Proposition 4.4 provides many examples in which the connected components of $\mathbb{E}_{d-1}(\alpha)$ are topologically nontrivial. In contrast, Theorem 3.7 implies that components of $\mathbb{E}_{1}(\alpha)$ are always contractible, as they are intersections of open hemispheres, which are geodesically convex.

\section{REgularity}

Although we cannot do justice to the extensive literature on expansiveness, we will indicate several ways in which it has appeared as a significant dynamical condition. We recommend [H2] for an extensive review. Below, by "homeomorphism" we will mean a homeomorphism of a compact metric space to itself.

Expansiveness is transparently a geometric condition, and one precise aspect of this is that expansive homeomorphisms admit hyperbolic metrics [Fr1, R]. Expansiveness is also a topological condition: various topological spaces [H2, Ka], for example the 2-sphere, admit no expansive homeomorphism [Lew, H1], and the domain of an expansive homeomorphism must be zero-dimensional if it is minimal 
[Man1] or has zero entropy [Fa]. Expansiveness is a finiteness condition: an expansive homeomorphism can only be defined on a finite dimensional space [Man1]; an expansive homeomorphism of a compact surface must be pseudo-Anosov [Lew, H1]; any expansive system is a quotient of a subshift $X$ by a map whose equivalence relation is the intersection of $X \times X$ with a shift of finite type [Fr4]; an expansive system has finite entropy [W, Cor. 7.4.1] and only countably many automorphisms; an expansive homeomorphism has at least one measure of maximal entropy [W, Thm. 8.2], and it has a zeta function which under modest conditions must be rational [Fr3]. Expansiveness is an important algebraic condition in the study of the dynamics of automorphisms of compact groups [AM, La], or of actions generated by commuting automorphisms of compact groups [KS1].

What we want to emphasize now is that the expansiveness of a $\mathbb{Z}^{d}$-action along $k$-planes can be viewed as a strong regularity condition on the variation of dynamical properties and objects associated to the planes.

In order to make this more precise, we need to specify bases for the subspaces in $\mathbb{G}_{k}$. Let us call a $k$-tuple of linearly independent vectors in $\mathbb{R}^{d}$ a $k$-frame. Denote by $\mathbb{F}_{k}$ the set of all $k$-frames. There is a natural covering map $s_{k}: \mathbb{F}_{k} \rightarrow \mathbb{G}_{k}$ taking a $k$-frame to the $k$-plane it spans. The fiber of $s_{k}$ is isomorphic to $G L(k, \mathbb{R})$. For a $\mathbb{Z}^{d}$-action $\alpha$, let $\mathcal{C}$ be a connected component of $s_{k}^{-1}\left(\mathbb{E}_{k}(\alpha)\right)$. We will say that $\mathcal{C}$ is a expansive component of $k$-frames. Note that $s_{k}^{-1}\left(s_{k}(\mathcal{C})\right)$ consists of just two components, namely $\mathcal{C}$ itself and the set of $k$-frames in $\mathcal{C}$ with reversed orientation. If $k=1$, then an expansive component $\mathcal{C}$ of 1 -frames is an open cone in $\mathbb{R}^{d}$, and furthermore $\mathcal{C} \cap(-\mathcal{C})=\varnothing$ since any path in $\mathbb{R}^{d} \backslash\{\mathbf{0}\}$ from $\mathbf{v} \in \mathcal{C}$ to $-\mathbf{v}$ must intersect a nonexpansive $(d-1)$-plane.

As we will see below, certain dynamical invariants attached to $k$-frames vary nicely within $\mathcal{C}$, but can or must deteriorate at the boundary of $\mathcal{C}$, for example losing continuity, smoothness, or uniqueness. This abrupt change in passing from one expansive component to another is roughly like a "phase transition." Let us give some examples of this phenomenon.

Example 5.1 (Automorphisms of shifts of finite type). Let $\sigma: X \rightarrow X$ be a shift of finite type. An automorphism of $\sigma$ is a homeomorphism of $X$ commuting with $\sigma$. See [BLR] for an extensive discussion of the group of such automorphisms. Their study has deepened recently, and has led to a counterexample to the reducible case of the shift equivalence conjecture [KRW, KR].

Consider the $\mathbb{Z}^{2}$-action $\alpha$ generated by an automorphism $\phi$ of a shift of finite type $\sigma$, so that $\alpha^{(1,0)}=\sigma$ and $\alpha^{(0,1)}=\phi$. Let $\mathcal{C}$ be the expansive component of 1 -frames containing $(1,0)$. As we show in $\S 8$, there are positive real algebraic numbers $\theta$ and $\xi$ such that for every $(m, n) \in \mathcal{C} \cap \mathbb{Z}^{2}$ the topological entropy of $\alpha^{(m, n)}$ is given by

$$
h\left(\alpha^{(m, n)}\right)=h\left(\sigma^{m} \phi^{n}\right)=m \log \theta+n \log \xi
$$

Moreover, there is an $r$ and $\left(\theta_{1}, \ldots, \theta_{r}\right),\left(\xi_{1}, \ldots, \xi_{r}\right) \in \mathbb{C}^{r}$ such that for every $(m, n) \in \mathcal{C} \cap \mathbb{Z}^{2}$ the zeta function of $\alpha^{(m, n)}$ is

$$
\zeta_{\alpha^{(m, n)}}(z)=\frac{1}{\prod_{j=1}^{r}\left(1-\theta_{j}^{m} \xi_{j}^{n} z\right)} .
$$

Even the shift equivalence classes of the shifts of finite type corresponding to these $\alpha^{(m, n)}$ can be nicely parameterized (Theorem 8.6, after [BK]). Observe that although the above formulas vary nicely in $\mathcal{C}$, they cannot possibly extend to all 
1-frames, since, for example, (5-1) would give negative entropies for some values of $m$ and $n$.

Nasu [N2] developed elaborate matrix techniques for studying such $\mathbb{Z}^{2}$-actions, especially the subdynamics of their Markov and expansive elements. One example [N2, Chap. 10, Example 1] is of particular interest here. This example constructs an automorphism $\phi$ of a shift $\sigma$ of finite type for which $\phi$ itself is a shift of finite type. Nasu shows that there are at least four nonexpansive lines for the resulting $\mathbb{Z}^{2}$-action, but the complete description of the nonexpansive set is not known. Let $\mathcal{C}$ be the expansive component containing $\sigma$ and $\mathcal{D}$ be the one containing $\phi$. For $\mathbf{m} \in \mathcal{C} \cap \mathbb{Z}^{2}$ the maps $\alpha^{\mathbf{m}}$ are all mixing shifts of finite type with a common measure $\mu_{\mathcal{C}}$ of maximal entropy, and similarly for $\mathcal{D}$. However, the measures $\mu_{\mathcal{C}}$ and $\mu_{\mathcal{D}}$ are quite different, one having a quadratic Perron eigenvalue while the other has a cubic Perron eigenvalue. Furthermore, the dimension groups associated to $\mathcal{C}$ and to $\mathcal{D}$ (see Theorem 8.6) are not isomorphic, one having rank two while the other has rank five.

Example 5.2 (Toral automorphisms). Let $A$ and $B$ be commuting (algebraic) expansive automorphisms of $\mathbb{T}^{n}$, and define a $\mathbb{Z}^{2}$-action $\alpha$ be $\alpha^{(1,0)}=A$ and $\alpha^{(0,1)}=$ $B$. The nonexpansive lines for $\alpha$ are those whose slope $s$ satisfies the condition that $A$ and $B$ have a common eigenspace with respective eigenvalues $\theta$ and $\xi$ such that $|\theta||\xi|^{s}=1$. Let $\mathcal{C}$ be a connected component of expansive 1-frames. For each common eigenspace of $A$ and $B$, it follows that for all $(m, n) \in \mathcal{C} \cap \mathbb{Z}^{2}$ the modulus of the corresponding eigenvalue of $A^{m} B^{n}=\alpha^{(m, n)}$ is either always $<1$ or always $>1$. We can then apply the standard formulas for entropy [W, Thm. 8.15] and zeta functions [Sma, Prop. 4.15] of toral automorphisms to obtain analogues of (5-1) and (5-2), valid for all $(m, n) \in \mathcal{C} \cap \mathbb{Z}^{2}$. For example, the $\theta$ and $\xi$ in (5-1) will be products of certain eigenvalues of $A$ and $B$, respectively, depending on $\mathcal{C}$. Other instances of this phenomenon are described in Examples 6.4 and 6.5. For more advanced examples of this sort, see [KaSp] and [Sma].

Using the Pesin theory of characteristic exponents [Pe], this analysis extends to a smooth $\mathbb{Z}^{d}$-action on a compact manifold preserving a measure that is absolutely continuous with respect to Lebesgue measure, showing that measure-theoretic entropy is linear on expansive components (this is described by Fried [Fr2], who attributes it to Ornstein).

Example 5.3 (Smooth hyperbolic actions). Katok and Spatzier [KaSp] studied the invariant measures of certain smooth hyperbolic actions of $\mathbb{Z}^{d}$ (and $\mathbb{R}^{d}$ and $\mathbb{N}^{d}$ ) which include the systems described in Example 5.2. A crucial step in their analysis was the introduction of Lyapunov hyperplanes and Weyl chambers, which for the systems of Example 5.2 are what we call the nonexpansive $(d-1)$-planes and the expansive components of 1-frames. They pointed out quite explicitly the importance of the Lyapunov hyperplanes in regulating the $\mathbb{Z}^{d}$ dynamics and the necessity of considering planes not spanned by integral points. A key aspect of this is a regularity condition: the action's stable and unstable distributions are constant on a Weyl chamber. We refer the reader to [KaSp] for a discussion of these ideas and their context.

Example 5.4 (Directional entropy). Let $\alpha$ be a $\mathbb{Z}^{d}$-action on a compact metric space. Milnor [Mi] defined both the topological and the measure-theoretic (for a given $\alpha$-invariant probability measure) directional entropies for $k$-planes in $\mathbb{R}^{d}$. His main interest was the $\mathbb{Z}^{d}$-action obtained from the time evolution of a $(d-$ 
1)-dimensional cellular automaton mapping. Here the $(d-1)$-dimensional directional entropies give a quantitative measure of "information flow" in the automaton. Even in this case, the $(d-1)$-dimensional directional entropy is not always a continuous function of the $(d-1)$-frame [Mi, Smi]. However, Milnor showed that it is continuous on "space-like" $(d-1)$ planes, from which it follows immediately that it is continuous on expansive components in $\mathbb{E}_{d-1}(\alpha)$. The measure-theoretic $(d-1)$-dimensional directional entropy is actually linear within expansive components (see [Mi, Thm. 4]). We devote $\S 6$ to extending these results.

Example 5.5 (Asymptotic foliations). Let $\alpha$ be a $\mathbb{Z}^{d}$-action on $(X, \rho)$, and $\mathbf{v}, \mathbf{w} \in$ $\mathbb{Z}^{d}$ lie in the same expansive component of 1 -frames. Then for all $x$ and $y$ in $X$, as either $n \rightarrow \infty$ or $n \rightarrow-\infty$, it turns out that

$$
\lim _{n \rightarrow \infty} \rho\left(\alpha^{n \mathbf{v}}(x), \alpha^{n \mathbf{v}}(y)\right)=0 \Longleftrightarrow \lim _{n \rightarrow \infty} \rho\left(\alpha^{n \mathbf{w}}(x), \alpha^{n \mathbf{w}}(y)\right)=0 .
$$

We can describe this situation by saying that $\alpha^{\mathbf{v}}$ and $\alpha^{\mathbf{w}}$ have the same stable foliation. This fact is exactly what underlies the regularity of the zeta function in an expansive component that we described in Example 5.1 [BK, Lemma 2.13] and Example 5.2 [Sma, Prop. 4.14]. It is also a significant ingredient in the work of Katok and Spatzier [KaSp].

\section{ENTROPY}

Let $\alpha$ be a $\mathbb{Z}^{d}$-action, and $1 \leq k \leq d$. Milnor [Mi] introduced a $k$-dimensional topological entropy function $\eta_{k}$. For each $\alpha$-invariant measure $\mu$ he also introduced a $k$-dimensional measure-theoretic entropy function $\eta_{k}^{\mu}$. These functions are defined for all compact sets in $\mathbb{R}^{d}$, and provide a rich class of invariants for $\alpha$. The expansive subdynamics of $\alpha$ constrains these lower dimensional entropy functions, and conversely.

Recall from the previous section that a $k$-frame is a $k$-tuple of linearly independent vectors in $\mathbb{R}^{d}$. Denote the line segment in $\mathbb{R}^{d}$ with endpoints $\mathbf{v}, \mathbf{w}$ by $[\mathbf{v}, \mathbf{w}]$. For each $k$-frame $\Phi=\left(\mathbf{v}_{1}, \ldots, \mathbf{v}_{k}\right)$, we let $Q_{\Phi}=\left[\mathbf{0}, \mathbf{v}_{1}\right] \oplus \cdots \oplus\left[\mathbf{0}, \mathbf{v}_{k}\right]$ denote the parallelepiped spanned by $\Phi$. Then the function $h_{k}(\Phi)=\eta_{k}\left(Q_{\Phi}\right)$ can be thought of as a $k$-dimensional "directional entropy" on the space $\mathbb{F}_{k}$ of $k$-frames. This function is not always continuous on $\mathbb{F}_{k}$ (see Example 6.6).

Let $\mathcal{C}$ be an expansive component of $\alpha$ in $\mathbb{F}_{k}$, as defined in the previous section. Extending work of Milnor, we show that $h_{k}$ is Lipschitz continuous for $\Phi \in \mathcal{C}$ (Theorem 6.9), and that for every $\alpha$-invariant measure $\mu$ the function $h_{k}^{\mu}(\Phi)=$ $\eta_{k}^{\mu}\left(Q_{\Phi}\right)$ is the restriction to $\mathcal{C}$ of a $k$-form on $\mathbb{R}^{d}$ (Theorem 6.16). Using this, we show that there is an $\alpha$-invariant measure that has maximal entropy for all $k$-frames in $\mathcal{C}$ if and only if the topological directional entropy function $h_{k}$ is multilinear on $\mathcal{C}$ (Theorem 6.25).

We conclude the section with an essentially complete description of the possible 1-dimensional topological entropy functions on expansive components of 1-frames for $\mathbb{Z}^{2}$-actions.

We start with the $k$-dimensional topological entropy function. The reader should consult [Mi] for additional motivation and examples.

Fix a $\mathbb{Z}^{d}$-action $\alpha$ on $(X, \rho)$. For a compact set $E \subset \mathbb{R}^{d}$ and $\epsilon>0$, define $N_{\alpha}(E, \epsilon)$ to be the cardinality of the smallest finite subset $Y \subset X$ such that for every $x \in X$ there is a $y \in Y$ with $\rho_{\alpha}^{E}(x, y)<\epsilon$. Recall that $E^{t}$ denotes the set 
of all vectors in $\mathbb{R}^{d}$ within distance $t$ of $E$. We use the notation $s E$ to denote $\{s \mathbf{v}: \mathbf{v} \in E\}$.

Definition 6.1. Let $E$ be a compact subset of $\mathbb{R}^{d}$ and $\epsilon>0$. Put

$$
\eta_{k}(E, \epsilon)=\sup _{t>0} \varlimsup_{s \rightarrow \infty} \frac{\log N_{\alpha}\left((s E)^{t}, \epsilon\right)}{s^{k}} .
$$

Define the $k$-dimensional topological entropy (with respect to $\alpha$ ) of $E$ to be

$$
\eta_{k}(E)=\lim _{\epsilon \rightarrow 0} \eta_{k}(E, \epsilon)
$$

For a $k$-frame $\Phi \in \mathbb{F}_{k}$, let $Q_{\Phi}$ be the parallelepiped spanned by $\Phi$. Define the $k$-dimensional topological directional entropy of $\Phi$ to be

$$
h_{k}(\Phi)=\eta_{k}\left(Q_{\Phi}\right) \text {. }
$$

The following summarizes some basic properties of $\eta_{k}$.

Theorem 6.2 (Milnor). The $k$-dimensional topological entropy function $\eta_{k}$ is monotone, subadditive, translation-invariant, and $k$-homogeneous; that is,

(1) $\eta_{k}(E) \leq \eta_{k}(F)$ if $E \subset F$ are compact sets,

(2) $\eta_{k}(E \cup F) \leq \eta_{k}(E)+\eta_{k}(F)$ for all compact sets $E$ and $F$,

(3) $\eta_{k}(E+\mathbf{v})=\eta_{k}(E)$ for all compact sets $E$ and all $\mathbf{v} \in \mathbb{R}^{d}$, and

(4) $\eta_{k}(s E)=s^{k} \eta_{k}(E)$ for all compact sets $E$ and all $s>0$.

Proof. The proofs are straightforward, and are discussed in [Mi, Thm. 1]. The only novelty is the possibility that $\eta_{k}$ is infinite, a case which is easily handled.

More can be said about $\eta_{k}$ when $\alpha$ has an expansive $k$-plane. This situation is closer to the spirit of Milnor's use of these notions to describe the subdynamics of cellular automata. In what follows, for each $k$-plane $V$ we let $\lambda_{V}$ denote $k$-dimensional Lebesgue measure on $V$, normalized so that the unit cube in $V$ (with respect to the inner product on $V$ inherited from $\mathbb{R}^{d}$ ) has $\lambda_{V}$-measure one. A subset of $\mathbb{R}^{d}$ is called polyhedral if it is a finite union of polyhedra.

Theorem 6.3 (Milnor). Suppose that $\alpha$ has an expansive $k$-plane. Then

(1) There is a constant $c$ such that $\eta_{k}(E) \leq c(\operatorname{diam} E)^{k}$ for every compact set $E \subset \mathbb{R}^{d}$. In particular, $\eta_{k}(E)<\infty$ for all compact sets $E$.

(2) For every $k$-plane $V$ there is a number $h_{k}(V)$ such that $\eta_{k}(E)=h_{k}(V) \lambda_{V}(E)$ for all compact sets $E \subset V$ for which $\lambda_{V}(\partial E)=0$.

(3) The numbers $h_{k}(V)$ are uniformly bounded from above as $V$ varies over all $k$-planes.

(4) If there is an expansive $k$-plane $V$ for which $h_{k}(V)=0$, then $h_{k}(W)=0$ for all $k$-planes $W$.

(5) For a compact polyhedral subset $E$ of a $k$-plane,

$$
\eta_{k}(E, \epsilon)=\sup _{t>0} \varliminf_{s \rightarrow \infty} \frac{\log N_{\alpha}\left((s E)^{t}, \epsilon\right)}{s^{k}} .
$$

Proof. The arguments from [Mi, Thm. 2] prove (2) and (5). Let $\delta$ be an expansive constant for $\alpha$. It follows easily from Proposition 3.8 that there is a constant $c$ such that for every $S \subset \mathbb{Z}^{d}$ with $|S| \geq 2$, we have that

$$
\log N_{\alpha}(S, \delta) \leq c(\operatorname{diam} S)^{k} .
$$




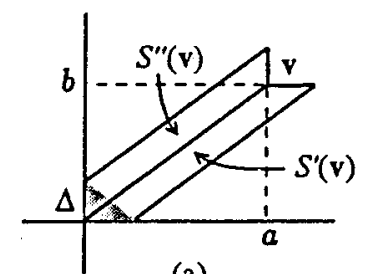

(a)

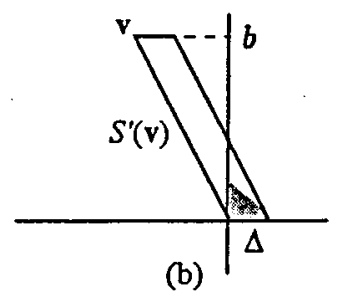

(b)

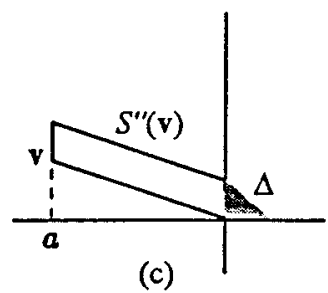

(c)

FigURE 7. Expansive strips to compute directional entropy.

In the terminology of [Mi], this means that the set function $S \mapsto \log N_{\alpha}(S, \delta)$ has growth degree at most $k$. It is also easy to verify that $\eta_{k}(E, \epsilon)=\eta_{k}(E, \delta)$ for all $\epsilon \leq \delta$. This completes the proof of (1). Now (3) follows from (1) and the observation that unit cubes in $k$-planes all have diameter $\sqrt{k}$. The argument for [Mi, Cor. 3] then applies to yield (4). Part (4) can also be proved by adapting arguments from [Sh].

Example 6.4. Let $\alpha$ be the $\mathbb{Z}^{2}$-action on $X$ discussed in Example 2.8. For $\theta \in$ $[0, \pi)$ let $L_{\theta}$ be the line making angle $\theta$ with the horizontal axis. We showed in Example 2.8 that $\mathbb{E}_{1}(\alpha)$ has three components, $C_{1}, C_{2}$, and $C_{3}$, corresponding respectively to $\theta$-ranges of $(0, \pi / 2),(\pi / 2,3 \pi / 4)$, and $(3 \pi / 4, \pi)$. Recall that $s_{1}: \mathbb{F}_{1} \rightarrow \mathbb{G}_{1}$ is the map from 1-frames (i.e., nonzero vectors) to the lines they generate. Then each $s_{1}^{-1}\left(C_{j}\right)$ has two connected components, one in the upper half plane and one in the lower. We let $\mathcal{C}_{j} \subset \mathbb{F}_{1}$ be the expansive component whose vectors are in the upper half-plane. We compute $h_{1}(\mathbf{v})=\eta_{1}([\mathbf{0}, \mathbf{v}])$ on these expansive components $\mathcal{C}_{j}$ of 1 -frames.

Fix a vector $\mathbf{v}=(a, b)$ in $\mathcal{C}_{1} \cup \mathcal{C}_{2} \cup \mathcal{C}_{3}$. Define strips

$$
S^{\prime}(\mathbf{v})=\left[\mathbf{0}, \mathbf{e}_{1}\right) \oplus[\mathbf{0}, \mathbf{v}], \quad S^{\prime \prime}(\mathbf{v})=\left(\mathbf{0}, \mathbf{e}_{2}\right] \oplus[\mathbf{0}, \mathbf{v}],
$$

and

$$
S(\mathbf{v})= \begin{cases}S^{\prime}(\mathbf{v}) \cup S^{\prime \prime}(\mathbf{v}) & \text { for } \mathbf{v} \in \mathcal{C}_{1}, \\ S^{\prime}(\mathbf{v}) & \text { for } \mathbf{v} \in \mathcal{C}_{2}, \\ S^{\prime \prime}(\mathbf{v}) & \text { for } \mathbf{v} \in \mathcal{C}_{3} .\end{cases}
$$

Then for each $\mathbf{v}$ the infinite extension $\widetilde{S}(\mathbf{v})$ of $S(\mathbf{v})$ is a half-open strip in direction $\mathbf{v}$ just wide enough to accommodate the unit simplex $\Delta$ (see Figure 7 ).

Now the coordinates of a point in $X$ can be chosen independently for all lattice points in $\widetilde{S}(\mathbf{v}) \cap \mathbb{Z}^{2}$, and all other coordinates are determined once this choice is made (see Figure 1). Since end effects become negligible, we see that

$$
\frac{1}{\log 2} h_{1}(\mathbf{v})=\lim _{t \rightarrow \infty} \frac{\left|S(t \mathbf{v}) \cap \mathbb{Z}^{2}\right|}{t} .
$$

Each horizontal line whose height is an integer between 0 and $t b$ intersects $S^{\prime}(t \mathbf{v})$ in exactly one lattice point, so that

$$
\left|S^{\prime}(t \mathbf{v}) \cap \mathbb{Z}^{2}\right|=[t b]+1 .
$$

Similarly,

$$
\left|S^{\prime \prime}(t \mathbf{v}) \cap \mathbb{Z}^{2}\right|=[t|a|]+1 .
$$


It follows that

$$
\frac{1}{\log 2} h_{1}(\mathbf{v})=\frac{1}{\log 2} h_{1}((a, b))= \begin{cases}a+b & \text { for } \mathbf{v} \in \mathcal{C}_{1}, \\ b & \text { for } \mathbf{v} \in \mathcal{C}_{2} \\ -a & \text { for } \mathbf{v} \in \mathcal{C}_{3}\end{cases}
$$

Observe that here $h_{1}$ is linear on each expansive component of 1-frames, but that this linear behavior changes abruptly (but continuously) when passing from one component to another. This calculation is given, for integral vectors, in [KS2].

Example 6.5. Let $\alpha$ be the $\mathbb{Z}^{2}$-action on the compact group $X$ described in Example 2.11, and use the same notations as there. Recall that $\alpha$ is the natural extension of multiplication by 2 and 3 on $\mathbb{T}$, and that locally $X$ looks like the direct product of a real interval, the 2 -adic integers $\mathbb{Z}_{2}$, and the 3 -adic integers $\mathbb{Z}_{3}$. The entropy of automorphisms of such groups was computed in [LW], where the use of " $p$-adic entropy" plays a role analogous to entropy for toral automorphisms. A consequence of this computation is that for $(m, n) \in \mathbb{Z}^{2}$,

$$
h\left(\alpha^{(m, n)}\right)=\log ^{+}\left|2^{m} 3^{n}\right|_{2}+\log ^{+}\left|2^{m} 3^{n}\right|_{3}+\log ^{+}\left|2^{m} 3^{n}\right|_{\infty},
$$

where $\log ^{+} t=\max \{0, \log t\}$ and $|\cdot|_{p}$ is the $p$-adic valuation. Here $\log ^{+}\left|2^{m} 3^{n}\right|_{p}$ measures the growth rate of $\alpha^{(m, n)}$ in the $p$-adic component (or the real interval if $p=\infty)$.

Recall that $\mathbb{N}_{1}(\alpha)$ consists of exactly three lines, making angles $0, \pi / 2$, and $\theta_{0}=\tan ^{-1}(-\log 2 / \log 3)$ with the positive horizontal axis. As in the previous example, this gives three expansive components $\mathcal{C}_{1}, \mathcal{C}_{2}$, and $\mathcal{C}_{3}$ in the upper half-plane corresponding to $\theta$-ranges $(0, \pi / 2),\left(\pi / 2, \theta_{0}\right)$, and $\left(\theta_{0}, \pi\right)$. It is easy to compute from $(6-1)$ that for $(m, n) \in \mathbb{Z}^{2}$ we have that

$$
h_{1}((m, n))= \begin{cases}m \log 2+n \log 3 & \text { for }(m, n) \in \mathcal{C}_{1}, \\ m \log 3 & \text { for }(m, n) \in \mathcal{C}_{2}, \\ -n \log 2 & \text { for }(m, n) \in \mathcal{C}_{3} .\end{cases}
$$

In Theorem 6.9 we show that $h_{1}$ is continuous on expansive components, from which it follows that $(6-2)$ is valid for all (not necessarily integral) vectors $(m, n)$. We are grateful to Tom Ward for pointing out this example.

The following example shows that directional entropy need not be continuous.

Example 6.6. Let $T: X \rightarrow X$ be an expansive homeomorphism with $h(T)>0$. There is a natural $\mathbb{Z}^{2}$-action $\alpha$ on $X \times \mathbb{Z}$ given by

$$
\alpha^{(m, n)}((x, j))=\left(T^{m} x, j+n\right) .
$$

Let $Y=(X \times \mathbb{Z}) \cup\{\infty\}$ be the one-point compactification of $X \times \mathbb{Z}$. There is a metric on $Y$ compatible with this topology, and $\alpha$ extends to a continuous $\mathbb{Z}^{2}$-action on $Y$ that we still call $\alpha$, having $\infty$ as a fixed point. Since $T$ is expansive, so is $\alpha$. However, $\mathbb{E}_{1}(\alpha)=\varnothing$.

Fix $\mathbf{v}=(a, b)$. If $b \neq 0$, then iterates of $\alpha$ in a strip in direction $\mathbf{v}$ converge to the point $\infty$, and it follows that $h_{1}(\mathbf{v})=0$. If $b=0$, then we are measuring the entropy of disjoint copies of $T$, so that $h_{1}((a, 0))=|a| h(T)>0$. Thus $h_{1}$ is discontinuous at the horizontal direction.

Milnor [Mi] gives more examples where $h_{1}$ is discontinuous, and where it is continuous but not convex (see also [Smi]). 
We begin our investigation of the continuity properties of $h_{k}$ on expansive components of $k$-frames. From now on in this section we will assume that $\alpha$ is expansive with expansive constant $\delta$.

Definition 6.7. Let $E$ and $F$ be compact subsets of $\mathbb{R}^{d}$. Say that $E$ shades $F$ if, for every $t>0$, there exists a $T>0$ such that $(s E)^{T} \operatorname{codes}(s F)^{t}$ for every $s>0$.

In Milnor's terminology, $E$ shades $F$ if $F$ is contained in the "umbra" of $E$ with respect to some causal cone [Mi, Lemma 4]. In Example 2.8, the base segment $E=\left[\mathbf{0}, \mathbf{e}_{1}\right]$ shades the whole simplex $F=\Delta$, since the coordinates in $s E$ of a point, plus a bounded amount of additional information to account for end effects, determine those in $s F$.

Proposition 6.8. Suppose that $E$ and $F$ are compact subsets of $\mathbb{R}^{d}$, and that $E$ shades $F$. Then $\eta_{k}(E \cup F)=\eta_{k}(E)$, and in particular $\eta_{k}(F) \leq \eta_{k}(E)$.

Proof. Since $\eta_{k}$ is monotone, $\eta_{k}(E) \leq \eta_{k}(E \cup F)$.

To prove the reverse inequality, fix $\epsilon>0$ and $t>0$. Since $\alpha$ is assumed expansive, there is a $t_{1}>t$ such that if $\rho_{\alpha}^{B\left(t_{1}\right)}(x, y)<\delta$ then $\rho_{\alpha}^{B(t)}(x, y)<\epsilon$. By the definition of shading, there is a $T>t_{1}$ such that $(s E)^{T}$ codes $(s F)^{t_{1}}$ for all $s>0$. Hence if $\rho_{\alpha}^{(s E)^{T}}(x, y)<\delta$, then $\rho_{\alpha}^{(s F)^{t_{1}}}(x, y)<\delta$, so that $\rho_{\alpha}^{(s F)^{t}}(x, y)<\epsilon$. If follows that

$$
N_{\alpha}\left((s E)^{T}, \delta\right) \geq N_{\alpha}\left((s(E \cup F))^{t}, \epsilon\right),
$$

and so $\eta_{k}(E) \geq \eta_{k}(E \cup F)$. The last statement now follows using monotonicity of $\eta_{k}$.

Using shading, we can now extend some basic inequalities and arguments due to Milnor for the case $k=d-1$ and $\mathbb{Z}^{d}$-actions generated by cellular automata. Recall that if $\Phi=\left(\mathbf{v}_{1}, \ldots, \mathbf{v}_{k}\right) \in \mathbb{F}_{k}$, we define $h_{k}(\Phi)=\eta_{k}\left(Q_{\Phi}\right)$. We also define $\|\Phi\|=\max _{j}\left\|\mathbf{v}_{j}\right\|$.

Theorem 6.9. Let $\mathcal{C}$ be an expansive component of $k$-frames for a $\mathbb{Z}^{d}$-action $\alpha$.

(1) If $\left(\mathbf{v}_{1}, \ldots, \mathbf{v}_{j}+\mathbf{w}, \ldots, \mathbf{v}_{k}\right) \in \mathcal{C}$, then $h_{k}\left(\mathbf{v}_{1}, \ldots, \mathbf{v}_{j}+\mathbf{w}, \ldots, \mathbf{v}_{k}\right) \leq h_{k}\left(\mathbf{v}_{1}, \ldots, \mathbf{v}_{j}, \ldots, \mathbf{v}_{k}\right)+h_{k}\left(\mathbf{v}_{1}, \ldots, \mathbf{w}, \ldots, \mathbf{v}_{k}\right)$.

(2) If $\left(\mathbf{v}_{1}, \ldots, \mathbf{v}_{j}, \ldots, \mathbf{v}_{k}\right) \in \mathcal{C}$, then

$h_{k}\left(\mathbf{v}_{1}, \ldots, \mathbf{v}_{j}+\mathbf{w}, \ldots, \mathbf{v}_{k}\right) \leq h_{k}\left(\mathbf{v}_{1}, \ldots, \mathbf{v}_{j}, \ldots, \mathbf{v}_{k}\right)+2 h_{k}\left(\mathbf{v}_{1}, \ldots, \mathbf{w}, \ldots, \mathbf{v}_{k}\right)$.

(3) If the linear span of $\mathbf{v}_{1}, \ldots, \mathbf{v}_{k}, \mathbf{w}$ contains an expansive $k$-plane, then

$h_{k}\left(\mathbf{v}_{1}, \ldots, \mathbf{v}_{j}+\mathbf{w}, \ldots, \mathbf{v}_{k}\right) \leq 2 h_{k}\left(\mathbf{v}_{1}, \ldots, \mathbf{v}_{j}, \ldots, \mathbf{v}_{k}\right)+2 h_{k}\left(\mathbf{v}_{1}, \ldots, \mathbf{w}, \ldots, \mathbf{v}_{k}\right)$.

(4) The restriction of $h_{k}$ to $\mathcal{C}$ is Lipschitz. If $k=1$ or $k=d-1$, then the restriction of $h_{k}$ to the closure of $\mathcal{C}$ is Lipschitz. In both cases, a Lipschitz constant for $h_{k}$ at a frame $\Phi$ can be chosen to be a function of $\|\Phi\|$ only.

Proof. When proving (1)-(3) we may assume that $j=1$. They are proved by finding appropriate shading relations on sets. First suppose that $k=1$, so that (1) becomes $h_{1}(\mathbf{v}+\mathbf{w}) \leq h_{1}(\mathbf{v})+h_{1}(\mathbf{w})$ provided that $\mathbf{v}+\mathbf{w} \in \mathcal{C}$. We claim that $[\mathbf{0}, \mathbf{v}] \cup[\mathbf{v}, \mathbf{v}+\mathbf{w}]$ shades $[\mathbf{0}, \mathbf{v}+\mathbf{w}]$ (see Figure $8(\mathrm{a})$ ). Let $L$ be the line through $\mathbf{v}+\mathbf{w}$, and suppose $r>0$. By Lemma 3.2, there are positive $t$ and $r_{0}$ such that $L^{t}\left(r_{0}\right)$ codes $L^{t+r}(0)$. Let $T=\sqrt{2} \max \left\{t, r_{0}, r\right\}$ and $E$ denote the triangle with vertices $\mathbf{0}, \mathbf{v}$, and $\mathbf{v}+\mathbf{w}$. For any $s>0$, we can inductively work from the vertex $s \mathbf{v}$ of $s E$ to show that $([s \mathbf{0}, s \mathbf{v}] \cup[s \mathbf{v}, s \mathbf{v}+s \mathbf{w}])^{T}$ successively codes larger and larger 


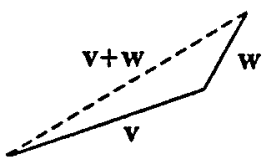

(a)

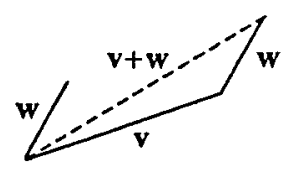

(b)

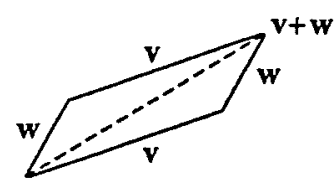

(c)

FiguRE 8. Shading relations for entropy inequalities.

parts of $(s E)^{r}$, stopping only when all of $(s E)^{r}$ is coded. This proves our shading claim. Then by Proposition 6.8 and Theorem 6.2(2),(3),

$$
\begin{aligned}
h_{1}(\mathbf{v}+\mathbf{w}) & =\eta_{1}([\mathbf{0}, \mathbf{v}+\mathbf{w}]) \leq \eta_{1}([\mathbf{0}, \mathbf{v}] \cup[\mathbf{v}, \mathbf{v}+\mathbf{w}]) \\
& \leq \eta_{1}([\mathbf{0}, \mathbf{v}])+\eta_{1}([\mathbf{0}, \mathbf{w}])=h_{1}(\mathbf{v})+h_{1}(\mathbf{w}) .
\end{aligned}
$$

We will now prove (1) when $k>1$ (and $j=1$ ). Let $\Psi=\left(\mathbf{v}_{2}, \ldots, \mathbf{v}_{k}\right)$ and $Q_{\Psi}$ denote $\left[\mathbf{0}, \mathbf{v}_{2}\right] \oplus \cdots \oplus\left[\mathbf{0}, \mathbf{v}_{k}\right]$ as usual. Then (1) becomes

$$
\eta_{k}\left(Q_{\mathbf{v}+\mathbf{w}} \oplus Q_{\Psi}\right) \leq \eta_{k}\left(Q_{\mathbf{v}} \oplus Q_{\Psi}\right)+\eta_{k}\left(Q_{\mathbf{w}} \oplus Q_{\Psi}\right)
$$

provided that $(\mathbf{v}+\mathbf{w}, \Psi) \in \mathcal{C}$. To prove $(6-3)$, consider the direct sum of Figure $8($ a) with $Q_{\Psi}$, and let $E$ denote the triangle with vertices $\mathbf{0}, \mathbf{v}$, and $\mathbf{v}+\mathbf{w}$. The same inductive argument as before shows that $Q_{\mathbf{v}+\mathbf{w}} \oplus Q_{\Psi}$ is shaded by

$$
\left(Q_{\mathbf{v}} \oplus Q_{\Psi}\right) \cup\left(\mathbf{v}+Q_{\mathbf{w}} \oplus Q_{\Psi}\right) \cup\left(E \oplus \partial Q_{\Psi}\right) .
$$

To handle the boundary term $E \oplus \partial Q_{\Psi}$, replace $\Psi$ by $s \Psi$ for large $s>0$. Since $\eta_{k}$ is proportional to Lebesgue measure on sets in a $(k-1)$-plane having measure zero boundary, it follows that

$s^{k-1} \eta_{k}\left(Q_{\mathbf{v}+\mathbf{w}} \oplus Q_{\Psi}\right) \leq s^{k-1} \eta_{k}\left(Q_{\mathbf{v}} \oplus Q_{\Psi}\right)+s^{k-1} \eta_{k}\left(Q_{\mathbf{w}} \oplus Q_{\Psi}\right)+s^{k-2} \eta_{k}\left(E \oplus \partial Q_{\Psi}\right)$.

Dividing by $s^{k-1}$ and letting $s \rightarrow \infty$ proves $(6-3)$.

The proofs of (2) and (3) are similar, using the shading relations depicted in Figure 8(b),(c).

To prove (4), first observe that by Theorem $6.3(2), \gamma=\sup \left\{h_{k}(V): V \in \mathbb{G}_{k}\right\}<$ $\infty$. Hence if $\Omega=\left(\mathbf{w}_{1}, \ldots \mathbf{w}_{k}\right)$ is a $k$-frame spanning a $k$-plane $W$, then

$$
h_{k}(\Omega) \leq \gamma \lambda_{W}\left(Q_{\Omega}\right) \leq \gamma\left\|\mathbf{w}_{1}\right\| \ldots\left\|\mathbf{w}_{k}\right\| .
$$

Throughout the rest of this proof we may assume that there is an $R>0$ such that all frames considered have entries with norm $\leq R$. Suppose that $\Phi=$ $\left(\mathbf{v}_{1}, \ldots, \mathbf{v}_{j} \ldots, \mathbf{v}_{k}\right) \in \mathcal{C}$, and let $\Psi=\left(\mathbf{v}_{1}, \ldots, \mathbf{v}_{j}+\mathbf{w}, \ldots, \mathbf{v}_{k}\right)$. Then by part $(1)$,

$$
h_{k}(\Phi) \leq h_{k}(\Psi)+h_{k}\left(\mathbf{v}_{1}, \ldots,-\mathbf{w}, \ldots, \mathbf{v}_{k}\right) \leq h_{k}(\Psi)+\gamma R^{k-1}\|\mathbf{w}\|
$$

while by part $(2)$

$$
h_{k}(\Phi) \geq h_{k}(\Psi)-2 h_{k}\left(\mathbf{v}_{1}, \ldots, \mathbf{w}, \ldots, \mathbf{v}_{k}\right) \geq h_{k}(\Psi)-2 \gamma R^{k-1}\|\mathbf{w}\| .
$$

We conclude that if $\Phi \in \mathcal{C}$, and $\Psi$ differs from $\Phi$ in only one coordinate by a vector $\mathbf{w}$, then $\left|h_{k}(\Phi)-h_{k}(\Psi)\right| \leq 2 \gamma R^{k-1}|| \mathbf{w} \|$.

Thus if $\Phi \in \mathcal{C}$ and $\|\Psi-\Phi\|$ is small enough, then by changing $\Phi$ into $\Psi$ one coordinate at a time we remain within $\mathcal{C}$, and our arguments apply to show that

$$
\left|h_{k}(\Psi)-h_{k}(\Phi)\right| \leq 2 k \gamma R^{k-1}|| \Psi-\Phi \| \text {. }
$$


This proves that $h_{k}$ is Lipschitz at $\Phi$, and that the Lipschitz constant depends only on $\|\Phi\|$.

Suppose next that $k=1, \mathbf{u} \in \partial \mathcal{C}$, and $\mathbf{v} \in \mathcal{C}$ is close to $\mathbf{u}$. Then the estimates $(6-4)$ and $(6-5)$ reduce to

$$
\left|h_{1}(\mathbf{u})-h_{1}(\mathbf{v})\right| \leq 2 \gamma\|\mathbf{u}-\mathbf{v}\|
$$

proving that $h_{1}$ is Lipschitz on $\overline{\mathcal{C}}$.

Finally, suppose that $k=d-1$. Let $\Psi \in \partial \mathcal{C}$, and $\Phi \in \mathcal{C}$ be close to $\Psi$. Since the $(d-1)$-planes spanned by $\Phi$ and $\Psi$ must intersect in a subspace of dimension at least $d-2$, there are $(d-1)$-frames $\Phi^{\prime}=\left(\mathbf{v}_{1}, \mathbf{v}_{2}, \ldots, \mathbf{v}_{k}\right)$ and $\Psi^{\prime}=$ $\left(\mathbf{v}_{1}+\mathbf{w}, \mathbf{v}_{2}, \ldots, \mathbf{v}_{k}\right)$ such that $\Phi$ and $\Phi^{\prime}$ span the same $(d-1)$-plane, $\Psi$ and $\Psi^{\prime}$ span the same $(d-1)$-plane, $h_{d-1}\left(\Phi^{\prime}\right)=h_{d-1}(\Phi), h_{d-1}\left(\Psi^{\prime}\right)=h_{d-1}(\Psi)$, and $\|\mathbf{w}\|$ is bounded by a constant times $\|\Phi-\Psi\|$. It then follows from (6-4) and (6-5) that $\left|h_{d-1}(\Phi)-h_{d-1}(\Psi)\right|$ is bounded by a constant times $\|\Phi-\Psi\|$, and that this constant depends only on $\|\Psi\|$.

Remark 6.10. (a) We know of no example with an expansive $k$-plane for which $h_{k}$ is not Lipschitz.

(b) Consider Example 6.6. Let $\mathbf{v}=(1,1)$ and $\mathbf{w}=(1,-1)$. Then $h_{1}(\mathbf{v}+\mathbf{w})>0$, while $h_{1}(\mathbf{v})=h_{1}(\mathbf{w})=0$. This shows that some assumption is needed for the inequality in part (3).

Incidentally, this provides a simple example of a pair $\phi=\alpha^{\mathbf{v}}, \psi=\alpha^{\mathbf{w}}$ of commuting homeomorphisms for which $h(\phi \circ \psi)>h(\phi)+h(\psi)$, showing that topological entropy is not in general subadditive. The first example of this phenomenon was discovered by Goodwyn [Go] and is more complicated.

We next turn to measure-theoretic $k$-dimensional entropy. Let $\mu$ be an $\alpha$ invariant Borel probability measure on $X$. Let $\mathcal{P}=\left\{P_{1}, \ldots, P_{m}\right\}$ denote a finite, measurable partition of $X$. Define

$$
H_{\mu}(\mathcal{P})=\sum_{j=1}^{m}-\mu\left(P_{j}\right) \log \mu\left(P_{j}\right)
$$

For a compact set $E \subset \mathbb{R}^{d}$, put

$$
H_{\mu}(E, \mathcal{P})=H_{\mu}\left(\bigvee_{\mathbf{n} \in E \cap \mathbb{Z}^{d}} \alpha^{-\mathbf{n}} \mathcal{P}\right)
$$

where $\bigvee$ denotes the common refinement of partitions.

Definition 6.11. Let $\mu$ be an $\alpha$-invariant probability measure, and $E \subset \mathbb{R}^{d}$ be compact. Let

$$
\eta_{k}^{\mu}(E, \mathcal{P})=\sup _{t>0} \varlimsup_{s \rightarrow \infty} \frac{H_{\mu}\left((s E)^{t}, \mathcal{P}\right)}{s^{k}} .
$$

Define the $k$-dimensional measure-theoretic entropy of $E$ (with respect to $\alpha$ and $\mu$ ) as

$$
\eta_{k}^{\mu}(E)=\sup _{\mathcal{P}} \eta_{k}^{\mu}(E, \mathcal{P}),
$$

where the supremum is over all finite measurable partitions $\mathcal{P}$ of $X$. Define the $k$-dimensional measure-theoretic directional entropy of a $k$-frame $\Phi$ to be

$$
h_{k}^{\mu}(\Phi)=\eta_{k}^{\mu}\left(Q_{\Phi}\right)
$$


Remark 6.12. It is important to point out that the usual argument for the continuity of $\eta_{k}^{\mu}(E, \mathcal{P})$ as a function of $\mathcal{P}$ requires the growth condition

$$
\sup _{t>0} \varlimsup_{s \rightarrow \infty} \frac{\left|(s E)^{t} \cap \mathbb{Z}^{d}\right|}{s^{k}}<\infty .
$$

For example, let $k<d, E$ be the $d$-dimensional unit cube, and $\mathcal{P}$ be a partition of $X$ with $H_{\mu}(\mathcal{P})<\epsilon$. Then clearly

$$
\frac{H_{\mu}\left((s E)^{t}, \mathcal{P}\right)}{s^{k}} \leq \frac{\left|(s E)^{t} \cap \mathbb{Z}^{d}\right|}{s^{k}} H_{\mu}(\mathcal{P}) \leq \epsilon s^{d-k},
$$

but this tells us nothing about $\eta_{k}^{\mu}(E, \mathcal{P})$. Indeed, we cannot rule out examples where $\eta_{k}^{\mu}(E, \mathcal{P})>\eta_{k}(E)$. We avoid these issues since our interest here is in $\eta_{k}^{\mu}(E, \mathcal{P})$ where $E$ is a $k$-dimensional polyhedral set. In the setting of [Mi] this issue does not arise since there $\eta_{k}^{\mu}(E)$ is defined as $\eta_{k}^{\mu}\left(E, \mathcal{P}_{0}\right)$, where $\mathcal{P}_{0}$ is the time-zero partition of $X$.

The proof of Theorem 6.16 requires several prelinary results. The first of these consists of measure-theoretic analogues of some earlier results for topological directional entropy.

Theorem 6.13. Let $\mu$ be an $\alpha$-invariant measure. Then properties (1) to (4) in Theorem 6.2 and properties (2) and (5) in Theorem 6.3 hold, with $\eta_{k}$ replaced by $\eta_{k}^{\mu}$ and $h_{k}$ replaced by $h_{k}^{\mu}$.

Proof. The proofs for the analogues of (1) to (4) in Theorem 6.2 are straightforward. The proof of [Mi, Thm. 2] works for the analogues of (2) and (5). The only novelty is the possibility $h_{k}^{\mu}=\infty$, which is an easy separate case. (Later we will see this case actually does not arise, and the analogues of (3) and (4) in Theorem 6.3 do hold.)

The compact sets we are interested in are described as follows.

Definition 6.14. A subset of $\mathbb{R}^{d}$ is called $k$-polyhedral if it is a finite union of polyhedra each of which has dimension at most $k$.

Proposition 6.15. Let $\alpha$ be an expansive $\mathbb{Z}^{d}$-action on $X$. Suppose that $D \subset \mathbb{R}^{d}$ is $k$-polyhedral and that $\left\{\mathcal{P}_{j}\right\}$ is an increasing sequence of measurable partitions which converges to the Borel $\sigma$-algebra on $X$. Then

$$
\eta_{k}^{\mu}(D)=\lim _{j \rightarrow \infty} \eta_{k}^{\mu}\left(D, \mathcal{P}_{j}\right)
$$

If $t>0$ is such that $\left\{\alpha^{-\mathbf{n}} \mathcal{P}: \mathbf{n} \in(\mathbb{R} D)^{t} \cap \mathbb{Z}^{d}\right\}$ generates the Borel $\sigma$-algebra on $X$, then

$$
\eta_{k}^{\mu}(D)=\varlimsup_{s \rightarrow \infty} \frac{H_{\mu}\left((s D)^{t}, \mathcal{P}\right)}{s^{k}} .
$$

Proof. Let $L=\lim _{j} \eta_{k}^{\mu}\left(D, \mathcal{P}_{j}\right)$. We first prove $L=\eta_{k}^{\mu}(D)$. Clearly $L \leq \eta_{k}^{\mu}(D)$, so without loss of generality we may assume $L$ is finite.

Since $D$ is $k$-polyhedral, for every $t>0$ there is a constant $c_{t}$ such that

$$
\left|(s D)^{t} \cap \mathbb{Z}^{d}\right| \leq c_{t} s^{k} \quad \text { for all } s \geq 1 .
$$


Suppose $\epsilon>0$ and $\mathcal{Q}$ is a finite measurable partition. Because $\left\{\mathcal{P}_{j}\right\}$ generates the Borel $\sigma$-algebra, we may pick $j \geq j_{0}$ such that $c_{t} H_{\mu}\left(\mathcal{Q} \mid \mathcal{P}_{j}\right)<\epsilon$. Observe that

$$
\begin{aligned}
H_{\mu}\left((s D)^{t}, \mathcal{Q}\right) & \leq H_{\mu}\left((s D)^{t}, \mathcal{Q} \vee \mathcal{P}_{j}\right) \\
& \leq H_{\mu}\left((s D)^{t}, \mathcal{P}_{j}\right)+\left|(s D)^{t} \cap \mathbb{Z}^{d}\right| H_{\mu}\left(\mathcal{Q} \mid \mathcal{P}_{j}\right) \\
& \leq H_{\mu}\left((s D)^{t}, \mathcal{P}_{j}\right)+c_{t} s^{k} H_{\mu}\left(\mathcal{Q} \mid \mathcal{P}_{j}\right) .
\end{aligned}
$$

Divide by $s^{k}$, take the $\lim \sup$ as $s \rightarrow \infty$, and take the sup over $t>0$ to obtain

$$
\eta_{k}^{\mu}(D, \mathcal{Q}) \leq \eta_{k}^{\mu}\left(D, \mathcal{P}_{j}\right)+\epsilon .
$$

Now let $\eta_{k}^{\mu}\left(D, \mathcal{P}_{j}\right)$ increase to $L$, let $\epsilon$ go to zero, and take the sup over $\mathcal{Q}$ to deduce $\eta_{k}^{\mu}(D) \leq L$. This proves $\eta_{k}^{\mu}(D)=L$.

The last claim is now routine.

A $k$-form on $\mathbb{R}^{d}$ is a $k$-multilinear skew-symmetric function from $\left(\mathbb{R}^{d}\right)^{k}$ to $\mathbb{R}$. Our next goal is to show that on each expansive component $h_{k}^{\mu}$ is given by a $k$-form.

Theorem 6.16. Let $\mathcal{C}$ be an expansive component of $k$-frames for $\alpha$ and $\mu$ be an $\alpha$-invariant measure. Then there is a $k$-form $\omega$ on $\mathbb{R}^{d}$ such that $h_{k}^{\mu}$ and $\omega$ agree on $\mathcal{C}$.

The following measure version of Proposition 6.8 is at the heart of our results on measure theoretic directional entropy.

Proposition 6.17. Let $\alpha$ be an expansive $\mathbb{Z}^{d}$-action. Suppose that $E$ and $F$ are $k$-polyhedral sets in $\mathbb{R}^{d}$ and that $E$ shades $F$. Then

$$
\eta_{k}^{\mu}(E \cup F)=\eta_{k}^{\mu}(E) .
$$

Proof. It is easy to construct an increasing sequence $\left\{\mathcal{P}_{j}\right\}$ of partitions of $X$ with $\max _{P \in \mathcal{P}_{j}} \operatorname{diam}(P) \rightarrow 0$ as $j \rightarrow \infty$ and with

$$
\mu\left(\bigcup_{P \in \mathcal{P}_{j}} \partial P\right)=0 \quad \text { for all } j
$$

(see [W, Thm. 8.3]). It follows that the $\mathcal{P}_{j}$ increase to the Borel $\sigma$-algebra.

Let us fix one such partition $\mathcal{P}=\left\{P_{1}, \ldots, P_{m}\right\}$ for which $\max _{i} \operatorname{diam}\left(P_{i}\right)<\delta$, the expansive constant for $\alpha$. Given $\xi>0$, let

$$
P_{i}^{\prime}=\left\{x \in P_{i}: \operatorname{dist}\left(x, \partial P_{i}\right) \leq \xi\right\},
$$

$P_{0}^{\prime}=X \backslash \bigcup_{i=1}^{m} P_{i}^{\prime}$, and $\mathcal{P}_{\xi}^{\prime}=\left\{P_{0}^{\prime}, P_{1}^{\prime}, \ldots, P_{m}^{\prime}\right\}$. Then $\mu\left(P_{0}^{\prime}\right) \rightarrow 1$ as $\xi \rightarrow 0$, so that $H_{\mu}\left(\mathcal{P}_{\xi}^{\prime}\right) \rightarrow 0$ as $\xi \rightarrow 0$. Fix $\epsilon>0$. Since $F$ is $k$-polyhedral, for every $t>0$ there is a constant $c_{t}$ such that

$$
\left|(s F)^{t} \cap \mathbb{Z}^{d}\right| \leq c_{t} s^{k} \quad \text { for all } s \geq 1 .
$$

Now suppose $t>0$. Choose $\xi>0$ small enough that $H_{\mu}\left(\mathcal{P}_{\xi}^{\prime}\right)<c_{t} \epsilon$. Since $E$ shades $F$ and $\alpha$ is expansive, there is a $T>t$ such that

$$
\rho_{\alpha}^{(s E)^{T}}(x, y)<\delta \quad \text { implies that } \rho_{\alpha}^{(s F)^{t}}(x, y)<\xi .
$$

It follows that

$$
\left(\bigvee_{\mathbf{n} \in(s E \cup s F)^{t} \cap \mathbb{Z}^{d}} \alpha^{-\mathbf{n}} \mathcal{P}\right) \leq\left(\bigvee_{\mathbf{n} \in(s E)^{T} \cap \mathbb{Z}^{d}} \alpha^{-\mathbf{n}} \mathcal{P}\right) \vee\left(\bigvee_{\mathbf{n} \in(s F)^{t} \cap \mathbb{Z}^{d}} \alpha^{-\mathbf{n}} \mathcal{P}_{\xi}^{\prime}\right)
$$


Apply $H_{\mu}$, divide by $s^{k}$, take the lim sup as $s \rightarrow \infty$, and take the sup over $t>0$ to obtain

$$
\eta_{k}^{\mu}(E \cup F, \mathcal{P}) \leq \eta_{k}^{\mu}(E, \mathcal{P})+\epsilon .
$$

Letting $\epsilon \rightarrow 0$ shows that

$$
\eta_{k}^{\mu}(E \cup F, \mathcal{P}) \leq \eta_{k}^{\mu}(E, \mathcal{P})
$$

Replace $\mathcal{P}$ by $\mathcal{P}_{j}$, let $j \rightarrow \infty$, and apply the previous remarks to show that

$$
\eta_{k}^{\mu}(E \cup F)=\lim _{j \rightarrow \infty} \eta_{k}^{\mu}\left(E \cup F, \mathcal{P}_{j}\right) \leq \lim _{j \rightarrow \infty} \eta_{k}^{\mu}\left(E, \mathcal{P}_{j}\right)=\eta_{k}^{\mu}(E),
$$

completing the proof.

Remark 6.18. Suppose that $\Phi=\left(\mathbf{v}_{1}, \ldots, \mathbf{v}_{k}\right)$ is an expansive $k$-frame for $\alpha$ in which each vector is rational. Note that such a frame exists if $\alpha$ has any expansive $k$-frames since the set of expansive $k$-frames is open. For an integer $M>0$, observe that $\Phi$ is expansive if and only if $M \Phi=\left(M \mathbf{v}_{1}, \ldots, M \mathbf{v}_{k}\right)$ is expansive. Hence we may assume that $\Phi$ consists of integral vectors. Then define a $\mathbb{Z}^{k}$-action $\alpha_{\Phi}$ by

$$
\alpha_{\Phi}^{\left(n_{1}, \ldots, n_{k}\right)}=\alpha^{n_{1} \mathbf{v}_{1}+\cdots+n_{k} \mathbf{v}_{k}} .
$$

It is easy to check that $h_{k}(\Phi)$ and $h_{k}^{\mu}(\Phi)$ coincide with the usual $k$-dimensional topological and measure-theoretical entropies $h\left(\alpha_{\Phi}\right)$ and $h^{\mu}\left(\alpha_{\Phi}\right)$. In particular, the variational principle for $\mathbb{Z}^{k}$-actions [Mis] shows that $h_{k}(\Phi)=\sup _{\mu} h_{k}^{\mu}\left(\alpha_{\Phi}\right)$, where the supremum is over all $\alpha_{\Phi}$-invariant (but not necessarily $\alpha$-invariant) probability measures $\mu$. The $k$-homogeneity of $k$-dimensional directional entropy shows that this remains valid for rational expansive $k$-frames.

Theorem 6.19. Let $\alpha$ be a $\mathbb{Z}^{d}$-action having an expansive $k$-plane.

(1) If $h_{k}^{\mu}$ is zero at one expansive $k$-frame, then it is zero at every $k$-frame.

(2) The numbers $h_{k}^{\mu}(V)$ are uniformly bounded as $V$ ranges over all $k$-planes and $\mu$ ranges over all $\alpha$-invariant Borel probability measures.

(3) Suppose $\mu$ is an $\alpha$-invariant Borel probability. Then the statements of Theorem 6.9 are true with $h_{k}$ replaced by $h_{k}^{\mu}$.

Proof. Let $\Phi=\left(\mathbf{v}_{1}, \ldots, \mathbf{v}_{k}\right)$ be an expansive $k$-frame. Suppose $W$ is a $k$-plane and $\mu$ is an $\alpha$-invariant probability measure. For some $c>0$, the parallelepiped $c Q_{\Phi}$ shades a ball in $\mathbb{R}^{d}$ of radius $\sqrt{k}$, and therefore shades a cube with edge lengths 1 in $W$. Thus

$$
h_{k}^{\mu}(W) \leq c^{k} h_{k}^{\mu}(\Phi)
$$

With $h_{k}^{\mu}(\Phi)=0$, this proves (1). If $\Phi$ is a $k$-frame of rational vectors, then $c^{k} h_{k}^{\mu}(\Phi) \leq c^{k} h_{k}(\Phi)$. This gives an upper bound independent of $\mu$ and proves (2). The proof of (3) proceeds as in 6.9, with appeal to the the measure shading inequality 6.17 and boundedness result (2) above.

We define $\eta_{k}^{\mu}(E \mid F)$ to be $\eta_{k}^{\mu}(E \cup F)-\eta_{k}^{\mu}(F)$. The next proposition is essentially copied from Milnor [Mi, Cor. 2].

Proposition 6.20. Let $E$ and $F$ be $k$-polyhedral sets in $\mathbb{R}^{d}$. If $E \cap F$ is a polyhedral subset of a $k$-plane, then

$$
\eta_{k}^{\mu}(E \cup F) \leq \eta_{k}^{\mu}(E)+\eta_{k}^{\mu}(F)-\eta_{k}^{\mu}(E \cap F)
$$

Similarly, if $F_{0} \subset F$ is a polyhedral subset of a k-plane, then

$$
\eta_{k}^{\mu}(E \mid F) \leq \eta_{k}^{\mu}\left(E \mid F_{0}\right)
$$


Proof. The inequality (6-6) can also be written as

$$
\eta_{k}^{\mu}(E \cup F) \leq \eta_{k}^{\mu}(F)+\eta_{k}^{\mu}\left(E \cup F_{0}\right)-\eta_{k}^{\mu}\left(F_{0}\right) .
$$

On account of the subadditivity of the measure-theoretic conditional entropy of finite partitions, we have for all $t>0$ and $s>0$ that the corresponding inequality

$$
H_{\mu}\left((s E \cup s F)^{t}\right) \leq H_{\mu}\left((s F)^{t}\right)+H_{\mu}\left(\left(s E \cup s F_{0}\right)^{t}\right)-H_{\mu}\left(\left(s F_{0}\right)^{t}\right)
$$

is indeed satisfied. Hence we can divide by $s^{k}$, take $\overline{\lim }_{s \rightarrow \infty}$, and then take the supremum over $t>0$. Since the last term has a negative sign, to complete the argument we appeal to the analogue of (5) in 6.13 to replace the $\varlimsup$ lim with $\underline{\lim }$.

Remark 6.21. Note that the "entropy-correlation" of $E$ and $F$, defined by

$$
\eta_{k}^{\mu}(E)+\eta_{k}^{\mu}(F)-\eta_{k}^{\mu}(E \cup F)=\eta_{k}^{\mu}(E)-\eta_{k}^{\mu}(E \mid F) \geq 0,
$$

is symmetric in $E$ and $F$. Thus by (6-6), for $k$-polyhedral sets $E$ and $F$ the entropy-correlation can only decrease if either is replaced by a subset which is a polyhedral subset of a $k$-plane.

The next result shows that $\eta_{k}^{\mu}$ is "locally multi-additive" on an expansive component. By a cone, we mean a convex set closed under multiplication by positive scalars.

Lemma 6.22. Let $\mu$ be an $\alpha$-invariant measure, and $\Phi$ be an expansive $k$-frame for $\alpha$. Then $\Phi$ has as a neighborhood in $\mathbb{F}_{k}$ an open cone $\mathcal{N}$ on which $h_{k}^{\mu}$ is multi-additive wherever defined. That is, if

$$
\left(\mathbf{v}_{1}, \ldots, \mathbf{v}_{j}, \ldots, \mathbf{v}_{k}\right),\left(\mathbf{v}_{1}, \ldots, \mathbf{w}, \ldots, \mathbf{v}_{k}\right),\left(\mathbf{v}_{1}, \ldots, \mathbf{v}_{j}+\mathbf{w}, \ldots, \mathbf{v}_{k}\right) \in \mathcal{N}
$$

then

$$
h_{k}^{\mu}\left(\mathbf{v}_{1}, \ldots, \mathbf{v}_{j}+\mathbf{w}, \ldots, \mathbf{v}_{k}\right)=h_{k}^{\mu}\left(\mathbf{v}_{1}, \ldots, \mathbf{v}_{j}, \ldots, \mathbf{v}_{k}\right)+h_{k}^{\mu}\left(\mathbf{v}_{1}, \ldots, \mathbf{w}, \ldots, \mathbf{v}_{k}\right) .
$$

Proof. For brevity we shorten $h_{k}^{\mu}$ to $h$ and $\eta_{k}^{\mu}$ to $\eta$. We first consider the case $k=1$.

We can choose an open cone $\mathcal{N}$ containing $\Phi$ such that for any vectors (1-frames) $\mathbf{v}$ and $\mathbf{w}$ in $\mathcal{N}$, the set $[\mathbf{0}, \mathbf{v}] \cup[\mathbf{v}, \mathbf{v}+\mathbf{w}]$ shades $[\mathbf{0}, \mathbf{v}+\mathbf{w}]$, and vice versa. (This follows from Proposition 8.1.) Thus by the measurable shading result,

$\eta([\mathbf{0}, \mathbf{v}+\mathbf{w}])=\eta([\mathbf{0}, \mathbf{v}] \cup[\mathbf{v}, \mathbf{v}+\mathbf{w}]) \leq \eta([\mathbf{0}, \mathbf{v}])+\eta([\mathbf{v}, \mathbf{v}+\mathbf{w}])=\eta([\mathbf{0}, \mathbf{v}])+\eta([\mathbf{0}, \mathbf{w}])$ and by translation invariance of $\eta$ it suffices to prove $\eta([-\mathbf{v}, \mathbf{0}] \cup[\mathbf{0}, \mathbf{w}])=\eta([-\mathbf{v}, \mathbf{0}])$ $+\eta([\mathbf{0}, \mathbf{w}])$. Now for some $c>0,[-c \mathbf{w}, \mathbf{0}]$ shades $[-\mathbf{v}, \mathbf{0}]$, so that $\eta([-c \mathbf{w}, \mathbf{0}])=$ $\eta([-c \mathbf{w}, \mathbf{0}] \cup[-\mathbf{v}, \mathbf{0}])$. Since the entropy correlation of the sets $[-c \mathbf{w}, \mathbf{0}]$ and $[\mathbf{0}, \mathbf{w}]$ is zero, so is the entropy correlation of the sets $[-c \mathbf{w}, \mathbf{0}] \cup[-\mathbf{v}, \mathbf{0}]$ and $[\mathbf{0}, \mathbf{w}]$. By the previous proposition, the entropy correlation cannot increase on replacing $[-c \mathbf{w}, \mathbf{0}] \cup$ $[-\mathbf{v}, \mathbf{0}]$ with $[-\mathbf{v}, \mathbf{0}]$. Thus it remains zero, i.e. $\eta([-\mathbf{v}, \mathbf{0}] \cup[\mathbf{0}, \mathbf{w}])=\eta([-\mathbf{v}, \mathbf{0}])+$ $\eta([\mathbf{0}, \mathbf{w}])$.

The extension of the proof to $k>1$ is obtained by arguments similar to those of Theorem 6.9 .

Our final preparation shows that a "local form" is the restriction of a $k$-form. We let $G L^{+}(k, \mathbb{R})$ denote the group of $k \times k$ matrices with positive determinant. A 
matrix $A=\left[a_{i j}\right] \in G L^{+}(k, \mathbb{R})$ acts on $\left(\mathbb{R}^{d}\right)^{k}$ by

$$
A\left(\mathbf{v}_{1}, \ldots, \mathbf{v}_{k}\right)=\left(\sum_{j=1}^{k} a_{1 j} \mathbf{v}_{j}, \ldots, \sum_{j=1}^{k} a_{k j} \mathbf{v}_{j}\right) .
$$

Proposition 6.23. Suppose that $\mathcal{C}$ is a connected open subset of $\mathbb{F}_{k}$ that is invariant under $G L^{+}(k, \mathbb{R})$, and that $f: \mathcal{C} \rightarrow \mathbb{R}$ satisfies

(1) $f(A \Phi)=(\operatorname{det} A) f(\Phi)$ for all $A \in G L^{+}(k, \mathbb{R})$ and all $\Phi \in \mathcal{C}$,

(2) for every $\Phi \in \mathcal{C}$ there exists a neighborhood $\mathcal{N}$ of $\Phi$ in $\mathcal{C}$ on which $f$ is multi-additive wherever defined, in the sense of Lemma 6.22.

Then there is a unique $k$-form $\omega$ on $\left(\mathbb{R}^{d}\right)^{k}$ whose restriction to $\mathcal{C}$ is $f$.

Proof. For $\Phi=\left(\mathbf{v}_{1}, \ldots, \mathbf{v}_{k}\right) \in \mathcal{C}$, define $\omega_{\Phi}:\left(\mathbb{R}^{d}\right)^{k} \rightarrow \mathbb{R}$ by

$$
\omega_{\Phi}\left(\mathbf{x}_{1}, \ldots, \mathbf{x}_{k}\right)=\lim _{t \rightarrow \infty} \sum_{\Lambda \subset\{1, \ldots, k\}}(-1)^{k-|\Lambda|} f\left(t \mathbf{v}_{1}+\chi_{\Lambda}(1) \mathbf{x}_{1}, \ldots, t \mathbf{v}_{k}+\chi_{\Lambda}(k) \mathbf{x}_{k}\right),
$$

where $\chi_{\Lambda}$ is the indicator function of $\Lambda$. The sum in the limit is the same for all large $t$, so the limit is well-defined. Our assumptions on $f$ show that $\omega_{\Phi}$ is locally constant in $\Phi$, hence all the $\omega_{\Phi}$ are equal, say to $\omega$, since $\mathcal{C}$ is open and connected. It is easy to verify from its definition that $\omega$ is multilinear and restricts to $f$ on $\mathcal{C}$. Skew-symmetry follows from (1); e.g., when $k=2$, use $A=\left[\begin{array}{ll}1 & 1 \\ 0 & 1\end{array}\right]$ to obtain that $\omega(\mathbf{x}, \mathbf{x})=2 \omega(\mathbf{x}, \mathbf{x})$.

Proof of Theorem 6.16. Let $\mathcal{C}$ be an expansive component of $k$-frames. By Theorem $6.13, \eta_{k}^{\mu}$ is proportional to $k$-dimensional Lebesgue measure on subsets of a given $k$-plane having boundary of measure zero. It follows that for all $A \in G L^{+}(k, \mathbb{R})$,

$$
h_{k}^{\mu}(A \Phi)=(\operatorname{det} A) h_{k}^{\mu}(\Phi) \text {. }
$$

Lemma 6.22 shows that $h_{k}^{\mu}$ is locally multi-additive. Also, $\mathcal{C}$ is $G L^{+}(k, \mathbb{R})$-invariant. For if $\Phi \in \mathcal{C}$ and $A \in G L^{+}(k, \mathbb{R})$, then there is a path $\pi:[0,1] \rightarrow G L^{+}(k, \mathbb{R})$ with $\pi(0)=I, \pi(1)=A$, and $\operatorname{det} \pi(t)>0$ for $0 \leq t \leq 1$. Then $\pi(t) \Phi$ is expansive for all $t$ (they span the same expansive $k$-plane), so that $\pi(1) \Phi=A \Phi \in \mathcal{C}$. We can therefore apply Proposition 6.23 to complete the proof.

Let $\mathcal{C}$ be an expansive component of $k$-frames for $\alpha$. Our next goal is to find a criterion for the existence of an $\alpha$-invariant measure that is simultaneously maximal for all frames in $\mathcal{C}$. For this we need the following variational result.

Proposition 6.24. Let $\Phi$ be an expansive $k$-frame for a $\mathbb{Z}^{d}$-action $\alpha$. Then there is an $\alpha$-invariant measure $\mu$ such that $h_{k}^{\mu}(\Phi)=h_{k}(\Phi)$.

Proof. First suppose that $\Phi=\left(\mathbf{v}_{1}, \ldots, \mathbf{v}_{k}\right)$ is rational, i.e., each $\mathbf{v}_{j}$ has rational coordinates. For purposes of entropy, we can assume that the $\mathbf{v}_{j}$ are integral. Let $\alpha_{\Phi}$ denote the $\mathbb{Z}^{k}$-action generated by the $\alpha^{\mathbf{v}_{j}}$. Let $\mathcal{M}$ be the set of $\alpha_{\Phi}$-invariant probability measures $\mu$ for which $h_{k}^{\mu}(\Phi)=h_{k}(\Phi)$. Because $\alpha_{\Phi}$ is expansive, the map $\mu \mapsto h_{k}^{\mu}(\Phi)$ is upper semicontinuous. It follows from the variational principle for commuting maps [Mis] that $\mathcal{M}$ is compact and nonempty. By linearity of $\mu \mapsto h_{k}^{\mu}(\Phi)$, we see that $\mathcal{M}$ is also convex. Furthermore, $\mathcal{M}$ is clearly $\alpha$-invariant. By the Kakutani Fixed Point Theorem, there is a $\mu \in \mathcal{M}$ fixed by $\alpha$, and this $\mu$ is the required measure. 
Now suppose that $\Phi$ is not rational. Let $\left\{\Phi_{n}\right\}$ be a sequence of rational expansive $k$-frames converging to $\Phi$. By the previous paragraph, there are $\alpha$-invariant measures $\mu_{n}$ such that $h_{k}^{\mu_{n}}\left(\Phi_{n}\right)=h_{k}\left(\Phi_{n}\right)$ for $n \geq 1$. By passing to a subsequence if necessary, we may assume that $\left\{\mu_{n}\right\}$ converges weakly to a measure $\mu$, which is therefore also $\alpha$-invariant. Since $\alpha$ is expansive, it has a topological generator. Then a slight modification of the standard argument for upper semicontinuity shows that

$$
\varlimsup_{n \rightarrow \infty} h_{k}^{\mu_{n}}\left(\Phi_{n}\right) \leq h_{k}^{\mu}(\Phi) .
$$

By continuity of $h_{k}$ on $\mathcal{C}$,

$$
h_{k}^{\mu_{n}}\left(\Phi_{n}\right)=h_{k}\left(\Phi_{n}\right) \rightarrow h_{k}(\Phi),
$$

so that $h_{k}(\Phi) \leq h_{k}^{\mu}(\Phi)$. The reverse inequality is always true (Theorem 6.13).

The $\mathbb{Z}^{2}$-action $\alpha$ from Example 6.6 shows that this result can fail without the expansiveness assumption. For there $h_{1}\left(\mathbf{e}_{1}\right)>0$, while the only $\alpha$-invariant measure $\mu$ is the point mass at $\infty$, and for this measure $h_{1}^{\mu}\left(\mathbf{e}_{1}\right)=0$. In [Mi, Example 6.3] Milnor gives an example of a cellular automaton $\mathbb{Z}^{2}$-action for which the conclusion of Proposition 6.24 fails.

Theorem 6.25. Let $\mathcal{C}$ be an expansive component of $k$-frames for $\alpha$. Then there is an $\alpha$-invariant measure $\mu$ such that $h_{k}^{\mu}(\Phi)=h_{k}(\Phi)$ for all $\Phi \in \mathcal{C}$ if and only if $h_{k}$ is multilinear on $\mathcal{C}$.

Proof. The "if" part follows from Theorem 6.16. For the "only if" part, suppose that $h_{k}$ is multilinear on $\mathcal{C}$. Since $\eta_{k}$ is a multiple of $k$-dimensional Lebesgue measure for subsets of a fixed $k$-plane with measure zero boundary, it follows that $h_{k}$ is always skew-symmetric. Hence $h_{k}$ agrees with a $k$-form $\omega$ on $\mathcal{C}$. Fix $\Phi_{0} \in \mathcal{C}$. By Proposition 6.24, there is an $\alpha$-invariant measure $\mu$ such that $h_{k}^{\mu}\left(\Phi_{0}\right)=h_{k}\left(\Phi_{0}\right)$. By Theorem $6.16, h_{k}^{\mu}$ agrees with a $k$-form $\omega^{\mu}$ on $\mathcal{C}$. Now $\omega^{\mu}(\Psi) \leq \omega(\Psi)$ for all $\Psi \in \mathcal{C}$ by Theorem 6.13 , and $\omega^{\mu}\left(\Phi_{0}\right)=\omega\left(\Phi_{0}\right)$. One can verify that if two $k$-forms on $\mathbb{R}^{d}$ agree at a point in an open set $U$, and one dominates the other in $U$, then the forms must be equal. This establishes the result.

Here is another consequence of the variational result in Proposition 6.24.

Corollary 6.26. Let $\mathcal{C}$ be an expansive component of $k$-frames for $\alpha$. Suppose that $h_{k}$ is not identically zero. If $\Phi \in \mathcal{C}$ and $A \in G L(k, \mathbb{R})$ with $\operatorname{det} A<0$, then $A \Phi \notin \mathcal{C}$.

Proof. By Theorem 6.3(3), $h_{k}$ cannot vanish on $\mathcal{C}$. Let $\Phi \in \mathcal{C}$ and $A \in G L(k, \mathbb{R})$ with $\operatorname{det} A<0$. Proposition 6.24 shows that there is an $\alpha$-invariant measure $\mu$ such that $h_{k}^{\mu}(\Phi)=h_{k}(\Phi)>0$. If $A \Phi$ were also in $\mathcal{C}$, then since $h_{k}^{\mu}$ agrees with a $k$-form on $\mathcal{C}$ we would have that

$$
h_{k}^{\mu}(A \Phi)=(\operatorname{det} A) h_{k}^{\mu}(\Phi)<0,
$$

contradicting nonnegativity of $h_{k}^{\mu}$.

As pointed out previously, $h_{k}$ need not be continuous on $\mathbb{F}_{k}$. We do not know whether existence of an expansive $k$-plane forces continuity of $h_{k}$, or of $h_{k}^{\mu}$ for an $\alpha$-invariant measure $\mu$. The results of Sinai [Si1] and Park [P] show that for $\mathbb{Z}^{2}$-actions generated by cellular automata, $h_{1}^{\mu}$ is upper semicontinuous, but this appears to be the most that is known. 


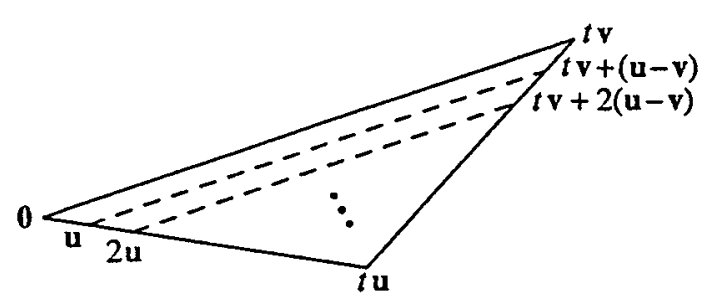

Figure 9. Coding most of a triangle.

We complete this section by determining the possible behaviors of $h_{1}$ on an expansive component $\mathcal{C}$ of 1 -frames for a $\mathbb{Z}^{2}$-action. Note that $\mathcal{C}$ is open by Theorem 3.7, and convex by Theorem 6.9 , so that $\mathcal{C}$ is an open cone in $\mathbb{R}^{2}$. Call $\mathcal{C}$ a proper cone if $\mathcal{C}$ is not an open half-plane. We first show that when $\mathcal{C}$ is a half-plane then $h_{1}$ is linear on $\mathcal{C}$.

Proposition 6.27. Suppose that $\alpha$ is a $\mathbb{Z}^{2}$-action having a single nonexpansive direction $L$. Then there is a linear functional $f$ on $\mathbb{R}^{2}$ whose kernel contains $L$, and for which $h_{1}(\mathbf{v})=|f(\mathbf{v})|$ for all $\mathbf{v} \in \mathbb{R}^{2}$.

Proof. Let $\mathcal{C}$ be one of the two half-planes in $\mathbb{R}^{2}$ with boundary $L$, so that $\mathcal{C}$ is an expansive component of 1 -frames. Let $\mu$ be any $\alpha$-invariant measure. By Theorem 6.16 , there is a linear functional $f_{\mu}$ such that $h_{1}^{\mu}(\mathbf{v})=f_{\mu}(\mathbf{v})$ for every $\mathbf{v} \in \mathcal{C}$. Since $h_{1} \geq 0$ on $\mathcal{C}$, it follows that $L \subset \operatorname{ker} f_{\mu}$. Consequently, $f=\sup _{\mu} f_{\mu}$, where the supremum is taken over all $\alpha$-invariant measures $\mu$, is a linear functional whose kernel contains $L$. By Proposition $6.24, h_{1}(\mathbf{v})=f(\mathbf{v})$ for all $\mathbf{v} \in \mathcal{C}$. Clearly $h_{1}(-\mathbf{v})=h_{1}(\mathbf{v})=|f(-\mathbf{v})|$ for all $\mathbf{v} \in \mathcal{C}$. The inequality (3) in Theorem 6.9 now shows that $h_{1}(\mathbf{u})=0$ for all $\mathbf{u} \in L$, completing the proof.

If $f$ is a linear functional on $\mathbb{R}^{2}$ whose kernel is a rational line $L$ it is easy to construct using a map of parameters (Example 2.12) a $\mathbb{Z}^{2}$-action $\alpha$ for which $h_{1}(\mathbf{v})=|f(\mathbf{v})|$, and whose expansive components are the two open half-planes bounded by $L$. Thus Proposition 6.27 completely characterizes the possible behavior of $h_{1}$ on an expansive component that is a half-plane whose boundary is rational. We do not know whether an expansive half-plane can have an irrational boundary (see Problem 9.2).

We now turn to the case of proper cones. For a vector $\mathbf{v} \in \mathbb{R}^{2}$ we let $Q_{\mathbf{v}}$ denote $[\mathbf{0}, \mathbf{v}]$, the "parallelepiped" spanned by the 1 -frame

$\mathbf{V}$.

Lemma 6.28. Let $\mathcal{C}$ be an expansive component of 1 -frames for a $\mathbb{Z}^{2}$-action. If $\mathbf{v} \in \mathcal{C}$ and $\mathbf{w} \in \mathcal{C} \cap(\mathbf{v}-\mathcal{C})$, then $Q_{\mathbf{v}}$ shades $Q_{\mathbf{w}}$.

Proof. For $\mathbf{x}, \mathbf{y} \in \mathbb{R}^{2}$ let $\Delta(\mathbf{x}, \mathbf{y})$ denote the triangle (including interior) with vertices $\mathbf{0}, \mathbf{x}$, and $\mathbf{y}$. Suppose that $\mathbf{v} \in \mathcal{C}$ and $\mathbf{w} \in \mathcal{C} \cap(\mathbf{v}-\mathcal{C})$. We may assume that $\mathbf{w}$ is not a multiple of $\mathbf{v}$. Since $\mathcal{C}$ is open, there is a $\mathbf{u} \in \mathcal{C} \cap(\mathbf{v}-\mathcal{C})$ such that the interior of $\Delta(\mathbf{u}, \mathbf{v})$ contains $\mathbf{w}$.

By Proposition 3.8, for every $\mathbf{x} \in \mathcal{C}$ there is a $\tau>0$ such that $[\mathbf{0}, \tau \mathbf{x}]$ shades every unit vector in some open cone containing $\mathbf{x}$. Since $[\mathbf{u}, \mathbf{v}]$ is compact, we can choose a $\tau$ such that $[\mathbf{0}, \tau \mathbf{v}]$ shades $[\mathbf{0}, \mathbf{u}]$. Because $-\mathbf{v}$ is in the expansive component $-\mathcal{C}$, and $\mathbf{u}-\mathbf{v} \in-\mathcal{C}$, we can also require that $[\mathbf{0},-\tau \mathbf{v}]$ shades $[\mathbf{0}, \mathbf{u}-\mathbf{v}]$. 
Consider $t \gg \tau$, and the triangle $\Delta(t \mathbf{u}, t \mathbf{v})$. The side $[\mathbf{0}, t \mathbf{v}]$ shades the convex hull of $\{\mathbf{0}, t \mathbf{v}, \mathbf{u}, t \mathbf{v}+(\mathbf{u}-\mathbf{v})\}$ (see Figure 9). The segment $[\mathbf{u}, t \mathbf{v}+(\mathbf{u}-\mathbf{v})]$ then shades the convex hull of $\{\mathbf{u}, t \mathbf{v}+(\mathbf{u}-\mathbf{v}), 2 \mathbf{u}, t \mathbf{v}+2(\mathbf{u}-\mathbf{v})\}$. Continuing this process, and using transitivity of the shading relation, we see that the side $[\mathbf{0}, t \mathbf{v}]=Q_{t \mathbf{v}}$ shades all of $\Delta(t \mathbf{u}, t \mathbf{v})$ except for a $\tau$-neighborhood of $t \mathbf{u}$. For large enough $t$ the segment $Q_{t \mathbf{w}}$ is shaded. Hence $Q_{\mathbf{v}}$ shades $Q_{\mathbf{w}}$.

The next proposition extends [Mi, Lem. 8].

Proposition 6.29. Let $\mathcal{C}$ be an expansive component of 1 -frames for a $\mathbb{Z}^{2}$-action $\alpha$, and assume that $h_{1}$ is not identically zero. If $\mathbf{v} \in \mathcal{C}$ and $\mathbf{w} \in \overline{\mathcal{C}}$, then

$$
0<h_{1}(\mathbf{v}) \leq h_{1}(\mathbf{v}+\mathbf{w})
$$

and the second inequality is strict if $\mathbf{w} \in \mathcal{C}$.

Proof. We may assume that $\mathbf{w} \neq \mathbf{0}$. Theorem 6.3(3) shows that $h_{1}$ does not vanish on any expansive 1-frame, establishing the first inequality. If $\mathbf{w} \in \mathcal{C}$, then the open set $\mathcal{C} \cap(\mathbf{v}+\mathbf{w}-\mathcal{C})$ contains $(1+\epsilon) \mathbf{v}$ for some $\epsilon>0$. By Lemma $6.28, Q_{\mathbf{v}+\mathbf{w}}$ shades $Q_{(1+\epsilon) \mathbf{v}}$. Hence

$$
h_{1}(\mathbf{v}+\mathbf{w}) \geq h_{1}((1+\epsilon) \mathbf{v})=(1+\epsilon) h_{1}(\mathbf{v})>h_{1}(\mathbf{v}) .
$$

Finally, if $\mathbf{w} \in \overline{\mathcal{C}}$, choose $\mathbf{w}_{n} \in \mathcal{C}$ converging to $\mathbf{w}$. By Theorem 6.9(4) and the above,

$$
h_{1}(\mathbf{v}+\mathbf{w})=\varlimsup_{n \rightarrow \infty} h_{1}\left(\mathbf{v}+\mathbf{w}_{n}\right) \geq h_{1}(\mathbf{v}) .
$$

It is convenient to name the inequality behavior from the previous proposition.

Definition 6.30. Let $\mathcal{C}$ be an open cone in $\mathbb{R}^{2}$. A function $\phi: \mathcal{C} \rightarrow \mathbb{R}$ is strictly increasing if whenever $\mathbf{v}, \mathbf{w} \in \mathcal{C}$, then $\phi(\mathbf{v})<\phi(\mathbf{v}+\mathbf{w})$. The function $\phi$ is homogeneous if $\phi(t \mathbf{v})=t \phi(\mathbf{v})$ for all $\mathbf{v} \in \mathcal{C}$ and all $t>0$.

The following elementary proposition, whose proof is straightforward, describes the class of functions we will use.

Proposition 6.31. Let $\mathcal{C}$ be a proper open cone in $\mathbb{R}^{2}$, and $\phi: \mathcal{C} \rightarrow \mathbb{R}$ be strictly positive, convex, and homogeneous. Let $D=\{\mathbf{u} \in \mathcal{C}: \phi(\mathbf{u})<1\}$. Then $\phi$ is strictly increasing if and only if there are linearly independent vectors $\mathbf{v}, \mathbf{w} \in \partial \mathcal{C}$ such that either

(1) $D=\{s \mathbf{v}+t \mathbf{w}: s>0$ and $0<t<1\}$, or

(2) $D$ is the interior of a compact convex subset of $[\mathbf{0}, \mathbf{v}] \oplus[\mathbf{0 w}]$ which contains $\mathbf{0}, \mathbf{v}$, and $\mathbf{w}$.

The point of this proposition is that when $\phi$ is strictly increasing, the set $D$ looks like Figure 10(a), not like (b).

We next introduce some notation needed for the next lemma. Let $x=\left(x_{n}\right) \in \mathbb{R}^{\mathbb{Z}}$. For $i \in \mathbb{Z}$ and $n \geq 1$, define

$$
\begin{aligned}
S_{i, n}(x) & =x_{i}+x_{i+1}+\cdots+x_{i+n-1} \\
\operatorname{osc}_{i, n}(x) & =\max _{1 \leq k \leq n} S_{i, k}(x)-\min _{1 \leq k \leq n} S_{i, k}(x), \\
\operatorname{osc}(x) & =\sup _{i, n} \operatorname{osc}_{i, n}(x) .
\end{aligned}
$$

We call osc $(x)$ the oscillation of $x$. 


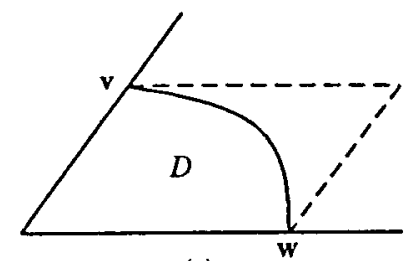

(a)

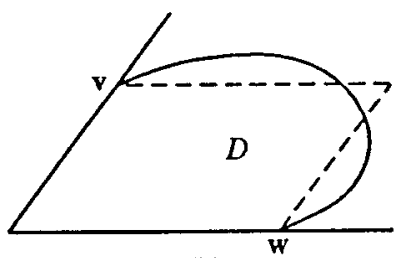

(b)

FiguRE 10. Unit balls for (a) strictly increasing and (b) not strictly increasing functions.

Lemma 6.32. Let $a$ and $b$ be real numbers such that $a / b$ is negative and irrational. Let $X$ be the subshift of $\{a, b\}^{\mathbb{Z}}$ consisting of all $x$ with $\operatorname{osc}(x) \leq|a|+|b|$. Then the shift on $X$ has entropy zero.

Proof. It is easy to check that $X$ is closed, nonempty, and invariant under the shift $\sigma$. By normalizing, we may assume that $b$ is irrational, that $b>0$, and that $|a|+|b|=b-a=1$. Hence the sequence $\{(n b): n \geq 1\}$ of fractional parts is uniformly distributed in $[0,1)$. Fix $\epsilon>0$. We can then find $N>0$ so that $\{(k b): 1 \leq k \leq N\}$ is $\epsilon$-dense in [0,1). Abbreviate $S_{1, k}$ to $S_{k}$. For $x \in X$ put

$$
m=m(x)=\min _{1 \leq k \leq N} S_{k}(x) \text { and } M=M(x)=\max _{1 \leq k \leq N} S_{k}(x) .
$$

Since $(k b) \equiv S_{k}(x)(\bmod 1)$, the choice of $N$ shows that $M-m \geq 1-2 \epsilon$. Since $\operatorname{osc}(x) \leq 1$, it follows that

$$
M-1 \leq S_{n+1}(x) \leq m+1 \text { for } n \geq N .
$$

If $x_{n+1}=b>0$, then $S_{n+1}(x)=S_{n}(x)+b \leq m+1$, so that

$$
S_{n}(x) \leq m+1-b .
$$

Similarly, if $x_{n+1}=a=b-1$, then

$$
S_{n}(x) \geq M-b .
$$

If both $x_{n+1}=a$ and $x_{n+1}=b$ are possible, then $S_{n}(x)$ must lie in the interval $[M-b, m+1-b]$, which has length $m+1-M \leq 2 \epsilon$. It follows that once $x_{1} \ldots x_{N}$ is fixed, each successive $x_{n+1}$ is determined by $x_{1} \ldots x_{n}$ except for a set of $n$ 's with frequency at most $2 \epsilon$. Since there are only finitely many $N$-blocks, we can conclude that

$$
\limsup _{n \rightarrow \infty} \frac{1}{n} \log \left|\left\{x_{1} \ldots x_{n}: x \in X\right\}\right| \leq(2 \epsilon) \log 2 .
$$

Since $\epsilon$ was arbitrary, it follows that $h(\sigma)=0$.

We remark that the shift space $X$ in this lemma is actually the orbit closure of a Sturmian sequence, giving an alternative proof for zero entropy.

We now come to the characterization of $h_{1}$ on proper expansive cones.

Theorem 6.33. Let $\mathcal{C}$ be a proper open cone in $\mathbb{R}^{2}$, and $\phi: \mathcal{C} \rightarrow \mathbb{R}$. The following conditions are equivalent.

(1) $\phi$ is strictly positive, strictly increasing, convex, and homogeneous.

(2) There exists a $\mathbb{Z}^{2}$-action on a compact metric space for which $h_{1}$ is not identically zero, and with $\mathcal{C}$ an expansive component of 1-frames such that $\phi$ and $h_{1}$ agree on $\mathcal{C}$. 
Proof. $(2) \Rightarrow(1)$ : Since $h_{1}$ is not identically zero, Proposition 6.29 shows that $h_{1}$ is strictly positive and strictly increasing. Theorem $6.9(1)$ shows that $h_{1}$ is convex, and Theorem 6.3(2) shows that $h_{1}$ is homogeneous.

(1) $\Rightarrow(2)$ : Let $D=\{\mathbf{u} \in \mathcal{C}: \phi(\mathbf{u})<1\}$, and first suppose that $D$ satisfies condition (2) in Proposition 6.31. Let $B=\partial D \backslash([\mathbf{0}, \mathbf{v}) \cup[\mathbf{0}, \mathbf{w}))$. By a map of parameters (Example 2.12), we may assume that $\mathbf{v}$ is in the second quadrant and $\mathbf{w}$ is in the fourth.

Since $\bar{D}$ is convex, we can find a family $\left\{L_{t}: 0 \leq t \leq 1\right\}$ of support lines for $B$ given by the equations $a_{t} x+b_{t} y=1$ such that

(i) $L_{0}$ contains $\mathbf{v}$ and is parallel to $\mathbf{w}$,

(ii) $L_{1}$ contains $\mathbf{w}$ and is parallel to $\mathbf{v}$,

(iii) $a_{t}$ is an increasing continuous nonnegative function, and

(iv) $b_{t}$ is a decreasing continuous nonnegative function.

Then $D$ is the intersection of the half-planes defined by $\mathbb{R} \mathbf{v}, \mathbb{R} \mathbf{w}$, and the $L_{t}$.

For our construction we use the family of $\beta$-shifts [IT]. For each real number $\beta>1$ there is a subshift $Y_{\beta}$ with alphabet $\{0,1, \ldots,[\beta]\}$ such that the entropy of the shift on $Y_{\beta}$ is $\log \beta$, and with $Y_{\beta_{1}} \subset Y_{\beta_{2}}$ whenever $\beta_{1} \leq \beta_{2}$. The $\beta$-shifts are usually defined as one-sided shifts, but we use their two-sided natural extensions.

Choose an interval $I=[a, b]$ that does not contain the slope of any vector in $\overline{\mathcal{C}}$. For each $u \in I$ we construct an action $\alpha_{u}^{\prime}$ on a space $X_{u}^{\prime}$ by modifying the construction in Proposition 4.1 as follows. The isolated nonexpansive line $K$ of that construction is $\mathbb{R} \mathbf{v}$. The only other nonexpansive line is the line $L$ of slope $u$. Choose the vectors $\mathbf{p}, \mathbf{q}$ used to define translation sequences such that the ratio $\left\|\pi_{K^{\perp}}(\mathbf{p})\right\| /\left\|\pi_{K^{\perp}}(\mathbf{q})\right\|$ is irrational. In condition (ii) of the definition of translation sequence, replace the right side with $\left\|\pi_{K^{\perp}}(\mathbf{p})\right\|+\left\|\pi_{K^{\perp}}(\mathbf{q})\right\|$. It follows from Lemma 6.32 that the subshift of translation sequences has zero entropy. Let $t(u)=(u-a) /(b-a)$, so that $t(u)$ runs through $[0,1]$ as $u$ runs through $I$. Given $u$, choose $\beta$ so that $\log \beta=a_{t(u)}$. Now define $X_{u}^{\prime} \subset\{*, 0,1, \ldots,[\beta]\}^{\mathbb{Z}^{2}}$ as follows. Given an $L$-strip $S$, a translation sequence $\mathcal{T}=\left\{\mathbf{k}_{j}\right\}$, and a point $y \in Y_{\beta}$, define

$$
x_{S, \mathcal{T}, y}(\mathbf{m})=\left\{\begin{array}{lc}
y_{m_{1}} & \text { if } \mathbf{m} \in \bigcup_{j \in \mathbb{Z}}\left(S+\mathbf{k}_{j}\right), \\
* & \text { otherwise. }
\end{array}\right.
$$

Let $X_{u}^{\prime}$ denote the union of all such points $x_{S, \mathcal{T}, y}$. Because the translation sequences are a subshift, it is easy to verify that $X_{u}^{\prime}$ is closed and $\mathbb{Z}^{2}$-invariant. Let $\alpha_{u}^{\prime}$ denote the restriction of the $\mathbb{Z}^{2}$-shift to $X_{u}^{\prime}$.

By Schwartzman's Theorem 3.9, the subshift of translation sequences must contain distinct points with the same past. One can then argue as in Proposition 4.1 that $\mathbb{N}_{1}\left(\alpha_{u}^{\prime}\right)=\{K, L\}$. Because the shift on translation sequences has zero entropy, the 1-dimensional directional entropy function of $\alpha_{u}^{\prime}$ is given by $(\xi, \eta) \mapsto \xi a_{t(u)}$.

Now define $X^{\prime}=\bigcup_{u \in I} X_{u}^{\prime}$ and $\alpha^{\prime}$ to be the restriction of the $\mathbb{Z}^{2}$-shift to $X^{\prime}$. Then $X^{\prime}$ is shift-invariant, and is closed since the functions $a_{t}$ and $b_{t}$ are monotone and the $\beta$-shifts are increasing with $\beta$. The nonexpansive lines for $\alpha^{\prime}$ are $\mathbb{R} \mathbf{v}$ and the lines whose slopes are in $I$.

Similarly we can define an action $\alpha^{\prime \prime}$ on $X^{\prime \prime}$ as a union of actions $\alpha_{u}^{\prime \prime}$ on $X_{u}^{\prime \prime}$, where the nonexpansive lines for $\alpha^{\prime \prime}$ are $\mathbb{R} \mathbf{w}$ and the lines whose slopes are in $I$, and with the directional entropy function of $\alpha_{u}^{\prime \prime}$ given by $(\xi, \eta) \mapsto \eta b_{t(u)}$. Let $X_{u}=X_{u}^{\prime} \times X_{u}^{\prime \prime}$, and $\alpha=\alpha^{\prime} \times \alpha^{\prime \prime}$ on $\bigcup_{u \in I} X_{u}$. Recall that if $T$ is a homeomorphism of a compact 
metric space $X$, and $X=\bigcup_{u \in I} X_{u}$ is the union of compact $T$-invariant sets $X_{u}$, then $h(T)=\sup h\left(\left.T\right|_{X_{u}}\right)$ (see [DGS, p. 139]). It follows that for rational vectors $(\xi, \eta) \in \mathcal{C}$, the directional entropy function $h_{1}$ for $\alpha$ is

$$
h_{1}(\xi, \eta)=\sup _{0 \leq t \leq 1}\left\{a_{t} \xi+b_{t} \eta\right\}
$$

Because $h_{1}$ is continuous in $\mathcal{C}$ and the functions $a_{t}, b_{t}$ are continuous, (6-10) holds for all $(\xi, \eta) \in \mathcal{C}$. Thus $D=\left\{\mathbf{w} \in \mathcal{C}: h_{1}(\mathbf{w})<1\right\}$, proving that $\phi$ and $h_{1}$ agree on $\mathcal{C}$. Also, $\mathbb{R} \mathbf{v}$ and $\mathbb{R} \mathbf{w}$ are nonexpansive, so that $\mathcal{C}$ is an expansive component for $\alpha$. This completes the case when $D$ satisfies condition (2) of Proposition 6.31.

If $D$ satisfies the alternative condition (1), we can use actions $\alpha_{u}^{\prime}$ as above, with nonexpansive lines $\mathbb{R} \mathbf{v}$ and $\mathbb{R} \mathbf{w}$, filling in $\mathbb{R} \mathbf{v}$-strips with symbols from a $\beta$-shift of entropy $\|\mathbf{u}\|$, completing this case, and the proof.

Remark 6.34. Given a proper cone $\mathcal{C}$, it is easy to modify the construction of the last paragraph in the previous proof to obtain an action $\alpha$ for which $h_{1} \equiv 0$ and having $\mathcal{C}$ as an expansive component. We have thus completely characterized the possible behaviors of $h_{1}$ on expansive components if $\mathbb{Z}^{2}$-actions that are proper cones.

Remark 6.35. For a $\mathbb{Z}^{d}$-action $\alpha$ preserving a measure $\mu$, Fried [Fr2] introduced the quantity $h_{*}^{\mu}(\alpha)$, defined as the reciprocal of the normalized volume of the unit ball for $h_{1}^{\mu}(\alpha)$ in $\mathbb{R}^{d}$. His motivation was to find a quantity reflecting entropy that does not always vanish for smooth actions. The quantity $h_{*}^{\mu}(\alpha)$ is an "integrated version" of the 1-dimensional measure-theoretic entropy function $h_{1}^{\mu}$.

\section{Algebraic examples}

Examples 2.8 and 2.9 are compact groups under coordinate-wise addition, and the shift actions are continuous group automorphisms. They are both cases of a rich class of algebraic examples constructed using commutative algebra, introduced by Kitchens and Schmidt [KS1] (see [S2] for a comprehensive account of this circle of ideas). In this section we will sketch this construction, determine completely the expansive subdynamics in certain cases, and establish a lower bound for the dimension of an expansive subspace. This bound involves the Krull dimension of a quotient ring, and estimating it from the number of generators in an associated ideal uses Krull's dimension theorem, called by Matsumura the most important result in the theory of commutative rings [Mt, p. xi].

We begin by describing the algebraic set-up. Let $R_{d}=\mathbb{Z}\left[u_{1}^{ \pm 1}, \ldots, u_{d}^{ \pm 1}\right]$ be the ring of Laurent polynomials in $d$ commuting variables. Let $M$ be an arbitrary countable $R_{d}$-module. Considering $M$ as an abelian group, we can form the compact abelian dual group, which we denote by $X_{M}$. Countability of $M$ is equivalent to metrizability of $X_{M}$. Multiplication by each of the coordinate variables $u_{i}$ is a group automorphism of $M$ since $u_{i}^{-1} \in R_{d}$, and these automorphisms commute. The corresponding dual automorphisms on $X_{M}$ then provide a $\mathbb{Z}^{d}$-action, denoted by $\alpha_{M}$. In this way every countable $R_{d}$-module $M$ gives rise to a $\mathbb{Z}^{d}$-action $\alpha_{M}$ on a compact metric group $X_{M}$.

This process can be reversed. Suppose that $\alpha$ is a $\mathbb{Z}^{d}$-action on a compact abelian metrizable group $X$. Let $M$ be the countable dual group. Then $M$ becomes an $R_{d}$-module by defining multiplication by $u_{i}$ to be the automorphism of $M$ dual to the $i$ th generating automorphism in $\alpha$. Thus the study of $\mathbb{Z}^{d}$-actions by group 
automorphisms can be transformed via duality to the study of $R_{d}$-modules, and the interplay of dynamics and commutative algebra gives this point of view its particular interest. This idea has been explored in a number of papers, e.g. [LSW], [S1], and [SW]. From this work it has emerged that the prime ideals associated to $M$ determine much of the dynamics of the $\mathbb{Z}^{d}$-action $\alpha_{M}$, just as the eigenvalues of a single toral automorphism determine much of its dynamics.

Let us see how Examples 2.8, 2.9, and 2.10 fit into this set-up. First consider $R_{d}$ as an $R_{d}$-module over itself. For $\mathbf{n}=\left(n_{1}, \ldots, n_{d}\right) \in \mathbb{Z}^{d}$ put $\mathbf{u}^{\mathbf{n}}=u_{1}^{n_{1}} u_{2}^{n_{2}} \ldots u_{d}^{n_{d}}$. Then as an abelian group, $R_{d}$ is the direct sum of $\mathbb{Z} \mathbf{u}^{\mathbf{n}}$ over $\mathbf{n} \in \mathbb{Z}^{d}$, so its dual group is the product group $\mathbb{T}^{\mathbb{Z}^{d}}$. Now let $d=2$, and consider the ideal $\mathfrak{a}=\left\langle 2,1+u_{1}+u_{2}\right\rangle$ in $R_{d}$. The quotient ring $M=R_{d} / \mathfrak{a}$ is an $R_{d}$-module, whose dual is the subgroup of $\mathbb{T}^{\mathbb{Z}^{2}}$ annihilated by $\mathfrak{a}$. Since $2 \in \mathfrak{a}$, each coordinate in a point in $X_{M}$ is annihilated by multiplication by 2 , so that $X_{M} \subset(\mathbb{Z} / 2 \mathbb{Z})^{\mathbb{Z}^{2}}$. The requirement that each point is annihilated by $1+u_{1}+u_{2}$ is exactly condition (2-2). Thus $\alpha_{M}=\alpha_{R_{2} /\left\langle 2,1+u_{1}+u_{2}\right\rangle}$ is the action discussed in Example 2.8. Similarly, Example 2.9 corresponds to the module $M=R_{3} /\left\langle 2,1+u_{1}+u_{2}+u_{3}\right\rangle$. It is also easy to verify that Example 2.10 corresponds to

$$
M=R_{2} /\left\langle\chi_{A}\left(u_{1}\right), \chi_{B}\left(u_{2}\right)\right\rangle
$$

where $\chi_{A}$ and $\chi_{B}$ are the characteristic polynomials of the matrices $A$ and $B$ in the example, and Example 2.11 to $M=R_{2} /\left\langle u_{1}-2, u_{2}-3\right\rangle$.

Mañé [Man1] proved that a compact metric domain of an expansive homeomorphism must be finite dimensional. A counterexample to the natural $\mathbb{Z}^{d}$ analogue of this theorem (pointed out to us by Tom Ward) can be described using another ideal.

Example 7.1. Let $d=2, \mathfrak{a}=\left\langle u_{2}^{2}-u_{2}-1\right\rangle$, and $M=R_{2} / \mathfrak{a}$. Then $X_{M} \cong\left(\mathbb{T}^{2}\right)^{\mathbb{Z}^{2}}$, $\alpha_{M}^{(0,1)}$ is the shift on $X_{M}$, and $\alpha_{M}^{(0,1)}=\ldots A \times A \times A \times \ldots$, where $A: \mathbb{T}^{2} \rightarrow \mathbb{T}^{2}$ has matrix

$$
A=\left[\begin{array}{ll}
0 & 1 \\
1 & 1
\end{array}\right]
$$

It is easy to verify directly that $\alpha_{M}$ is an expansive $\mathbb{Z}^{2}$-action, but the space $X_{M}$ on which it acts has infinite topological dimension.

To generalize Examples 2.8 and 2.9, let $p$ be a fixed rational prime and $f \in R_{d}$. Put $M=R_{d} /\langle p, f\rangle$. Our goal is to analyze the expansive subdynamics of $\alpha_{M}$.

First observe that if $f \in p R_{d}$, then $M=R_{d} /\langle p\rangle$. This case was treated in Example 2.7, where we showed that $\alpha_{M}$ is expansive, but $\mathbb{E}_{k}\left(\alpha_{M}\right)=\varnothing$ for $1 \leq k \leq$ $d-1$. Also, if $f \in c \mathbf{u}^{\mathbf{n}}+p R_{d}$, where $c \not \equiv 0(\bmod p)$, then $\langle p, f\rangle=R_{d}$, so in this case $X_{M}$ is a single point. We will therefore assume from now on that the reduction of $f$ modulo $p$ contains at least two nonzero terms.

For $\mathbf{n} \in \mathbb{Z}^{d}$, let $c_{\mathbf{n}}$ denote the coefficient of $\mathbf{u}^{\mathbf{n}}$ in $f$. Define the $\bmod p$ Newton polytope $\Delta(p, f)$ of $f$ to be the convex hull in $\mathbb{R}^{d}$ of those $\mathbf{n} \in \mathbb{Z}^{d}$ for which $c_{\mathbf{n}} \not \equiv 0$ $(\bmod p)$. This polytope has more than one point by our assumption on $f$. We will call $\Delta(p, f)$ nondegenerate if it is not contained in a translate of a $(d-1)$-plane.

Recall from Remark 3.11 that $\widetilde{\mathbb{G}}_{d-1}$ denotes the compact space of all oriented $(d-1)$-planes. Then $\widetilde{V} \in \widetilde{\mathbb{G}}_{d-1}$ is determined by its underlying $(d-1)$-plane $V$ together with an outward unit normal vector $\mathbf{v}$. Let $H_{\tilde{V}}$ denote the half-space spanned by $V$ and $[0, \infty) \mathbf{v}$. 
A support plane for $\Delta=\Delta(p, f)$ is a translate $\tau(V)$ of a $(d-1)$-plane containing at least one point of $\Delta$, and with all of $\Delta$ lying to one side of $\tau(V)$. An oriented support plane for $\Delta$ is a translate $\tau(\widetilde{V})$ of an oriented $(d-1)$-plane $\widetilde{V}$ such that $\tau(V)$ is a support plane for $\Delta$ and with $\Delta$ contained in $\tau\left(H_{\widetilde{V}}\right)$. For each $\widetilde{V} \in \widetilde{\mathbb{G}}_{d-1}$, there is a unique translate $\tau(\widetilde{V})$ that is an oriented support plane for $\Delta$. We let the bijection $\tau$ give the space of oriented support planes the topology of $\widetilde{\mathbb{G}}_{d-1}$.

The polytope $\Delta$ has a dual polytope $\Delta^{*}$. A concrete realization of $\Delta^{*}$ using polar sets is described in [G, §3.4]. This realization shows that the space of oriented support planes of $\Delta$ is homeomorphic to the $(d-1)$-skeleton of $\Delta^{*}$.

Let $\widetilde{V} \in \widetilde{\mathbb{G}}_{d-1}$ and $\tau(\widetilde{V})$ be the corresponding oriented support plane for $\Delta$. We say that $\Delta$ is $\widetilde{V}$-exposed if $\tau(V)$ contains exactly one point of $\Delta$, where $V$ is the underlying plane of $\widetilde{V}$. In Example 2.8, $p=2, f\left(u_{1}, u_{2}\right)=1+u_{1}+u_{2}, \Delta$ is the convex hull of $(0,0),(1,0)$, and $(0,1)$, and $\Delta$ is $\widetilde{V}$-exposed for all but three oriented lines $\widetilde{V}$, one for each side of the triangle $\Delta$. In general $\Delta$ is not $\widetilde{V}$-exposed exactly when the boundary of $\tau(\widetilde{V})$ contains a 1-dimensional edge of $\Delta$.

Recall from Remark 3.11 that $\widetilde{V} \in \widetilde{\mathbb{G}}_{d-1}$ is called a causal plane for $\alpha$ if $H_{\widetilde{V}}$ is an expansive set for $\alpha$.

Theorem 7.2. Let $p$ be a rational prime, $f$ be a polynomial in $R_{d}$ whose reduction $\bmod p$ has at least two terms, and $\Delta=\Delta(p, f)$ be the mod $p$ Newton polytope of $f$. Then $\widetilde{V} \in \widetilde{\mathbb{G}}_{d-1}$ is a causal plane for $\alpha=\alpha_{R_{d} /\langle p, f\rangle}$ if and only if $\Delta$ is $\widetilde{V}$-exposed.

If $\Delta$ is nondegenerate, then the space of non-causal planes in $\widetilde{\mathbb{G}}_{d-1}$ is homeomorphic to the $(d-2)$-skeleton of the dual polytope $\Delta^{*}$.

Proof. Let $E=\left\{\mathbf{n} \in \mathbb{Z}^{d}: c_{\mathbf{n}} \not \equiv 0(\bmod p)\right\}$. Then $X=X_{R_{d} /\langle p, f\rangle}$ is the set of those $x \in(\mathbb{Z} / p \mathbb{Z})^{\mathbb{Z}^{d}}$ for which

$$
\sum_{\mathbf{n} \in E} c_{\mathbf{n}} x(\mathbf{n}+\mathbf{k}) \equiv 0 \quad(\bmod p)
$$

for all $\mathbf{k} \in \mathbb{Z}^{d}$. By definition, $\Delta$ is the convex hull of $E$, and it contains more than one point by our assumption on $f$.

Suppose that $\widetilde{V} \in \widetilde{\mathbb{G}}_{d-1}$, and that $\Delta$ is not $\widetilde{V}$-exposed. Then $\tau(\widetilde{V})$ contains at least two points of $E$. As in Example 2.8, we can inductively construct a nonzero point $x$ in $X$ all of whose coordinates in $\tau(\widetilde{V})$ are 0 . This shows that $\widetilde{V}$ is not a causal plane for $\alpha$.

Conversely, suppose that $\widetilde{V} \in \widetilde{\mathbb{G}}_{d-1}$ and $\Delta$ is $\widetilde{V}$-exposed. Let $\mathbf{w}$ be the unit normal to $V$ which does not lie in $\widetilde{V}$. Choose $\epsilon>0$ such that $(-\epsilon) w+\tau(\widetilde{V})$ contains all points of $\mathbb{Z}^{d} \cap \Delta$ except the unique point $\mathbf{m}$ of $\Delta$ on the boundary of $\tau(\widetilde{V})$. Let $\widetilde{V}_{t}$ denote $\widetilde{V}+t w$ and suppose $\mathbf{k} \in\left(\widetilde{V}^{t+\epsilon} \backslash \widetilde{V}^{t}\right) \cap \mathbb{Z}^{d}$. Since $c_{\mathbf{m}}$ is invertible $(\bmod p)$, the condition

$$
\sum_{\mathbf{n} \in E} c_{\mathbf{n}} x(\mathbf{n}+\mathbf{k}-\mathbf{m}) \equiv 0 \quad(\bmod p)
$$

shows we can compute $x(\mathbf{k})$ from the value of the $x(\mathbf{n})$ with $\mathbf{n} \in V_{t}$. This completes the proof that $\widetilde{V}$ is a causal plane.

Finally, suppose that $\Delta$ is nondegenerate. It is straightforward to use the polar set realization of $\Delta^{*}$ given in $[\mathrm{G}, \S 3.4]$ to show that the set of oriented support 
planes of $\Delta$ that contain an edge of $\Delta$ is homeomorphic to the $(d-2)$-skeleton of $\Delta^{*}$.

For $V \in \mathbb{G}_{d-1}$, we say that $\Delta$ is $V$-exposed if each support plane parallel to $V$ contains exactly one point of $\Delta$. Recall that $p_{d-1}: \widetilde{\mathbb{G}}_{d-1} \rightarrow \mathbb{G}_{d-1}$ is the covering map that forgets orientation.

Theorem 7.3. Let $p$ be a rational prime, $f$ be a polynomial in $R_{d}$ whose reduction $\bmod p$ has at least two terms, and $\Delta=\Delta(p, f)$ be the $\bmod p$ Newton polytope of $f$. Then $V \in \mathbb{G}_{d-1}$ is expansive for $\alpha=\alpha_{R_{d} /\langle p, f\rangle}$ if and only if $\Delta$ is $V$-exposed. All subspaces of $\mathbb{R}^{d}$ with dimension $\leq d-2$ are nonexpansive for $\alpha$.

If $\Delta$ is nondegenerate, then $\mathbb{N}_{d-1}(\alpha)$ is the image under the covering map $p_{d-1}$ of a set homeomorphic to the $(d-2)$-skeleton of the dual polytope $\Delta^{*}$.

Proof. Recall from Remark 3.11 that $V \in \mathbb{G}_{d-1}$ is expansive if and only if both its oriented versions in $p_{d-1}^{-1}(V)$ are causal planes. By Theorem 7.2, this is equivalent to $\Delta$ being $V$-exposed.

Suppose that $U$ is a subspace with dimension $\leq d-2$. Since $\Delta$ contains nontrivial 1-dimensional edges, we can increase $U$ to a $(d-1)$-dimensional subspace $V$ that contains a translate of an edge of $\Delta$. Then $\Delta$ is not $V$-exposed, so that $V$, and hence $U$, is not expansive for $\alpha$.

Finally, recall from Remark 3.11 that the nonexpansive $(d-1)$-planes are the image of the non-causal planes under the map $p_{d-1}$. Then the last claim follows from Theorem 7.2.

In our previous examples, subspaces stop being expansive when their dimension is small enough. Let us introduce a quantity to reflect this idea.

Definition 7.4. Let $\alpha$ be a $\mathbb{Z}^{d}$-action on a compact metric space. Define the expansive rank $\operatorname{erk}(\alpha)$ of $\alpha$ to be the smallest $k$ for which there is an expansive subspace for $\alpha$ with dimension $k$. If $\alpha$ is not expansive, by convention we put $\operatorname{erk}(\alpha)=d+1$.

For instance, Example 2.7 shows that if $M=R_{d} /\langle k\rangle$ for some integer $k$, then $\operatorname{erk}\left(\alpha_{M}\right)=d$. The previous theorem provides examples of the form $M=R_{d} /\langle p, f\rangle$ for which $\operatorname{erk}(\alpha)=d-1$. We will show below that, roughly speaking, the more polynomials needed to generate an ideal $\mathfrak{a}$, the smaller is $\operatorname{erk}\left(\alpha_{R_{d} / \mathfrak{a}}\right)$.

In order to quantify this idea, recall that the Krull dimension of a ring is the length of the longest strictly increasing chain of prime ideals in the ring. We denote the Krull dimension of a ring $R$ by $\operatorname{kdim} R$. For example, in $R_{d}$ the chain

$$
0 \subset\langle 2\rangle \subset\left\langle 2, u_{1}\right\rangle \subset\left\langle 2, u_{1}, u_{2}\right\rangle \subset \cdots \subset\left\langle 2, u_{1}, u_{2}, \ldots, u_{d}\right\rangle
$$

of prime ideals has length $d+1$ and it is known that there is no longer chain, so that $\operatorname{kdim} R_{d}=d+1$. We use Krull dimension to estimate the expansive rank of some algebraic examples.

Theorem 7.5. Let $\mathfrak{a}$ be an ideal in $R_{d}$ that can be generated by $g$ elements. Then

$$
\operatorname{erk}\left(\alpha_{R_{d} / \mathfrak{a}}\right) \geq \operatorname{kdim} R_{d} / \mathfrak{a}-1 \geq d-g .
$$

If one of the $g$ generators of $\mathfrak{a}$ can be chosen to be a rational prime, then

$$
\operatorname{erk}\left(\alpha_{R_{d} / \mathfrak{a}}\right) \geq d-g+1 .
$$


Proof. Call a subspace of $\mathbb{R}^{d}$ rational if it has a basis of integral vectors. The set of rational $k$-dimensional subspaces is clearly dense in $\mathbb{G}_{k}$. Since $\mathbb{E}_{k}(\alpha)$ is open, we may choose a rational subspace $V$ whose $\operatorname{dimension}$ is $\operatorname{erk}\left(\alpha_{R_{d} / \mathfrak{a}}\right)$.

Suppose that $V \in \mathbb{G}_{k}$ is expansive for $\alpha_{R_{d} / \mathfrak{a}}$, and that $V$ is spanned by vectors $\mathbf{n}_{1}, \ldots, \mathbf{n}_{k} \in \mathbb{Z}^{d}$. Let $R_{k}=\mathbb{Z}\left[v_{1}^{ \pm 1}, \ldots, v_{k}^{ \pm 1}\right]$. We can consider $R_{d} / \mathfrak{a}$ as an $R_{k}$-module by the rule $v_{j}(f+\mathfrak{a})=\mathbf{u}^{\mathbf{n}_{j}} f+\mathfrak{a}$. Since $V$ is expansive, we must have that $R_{d} / \mathfrak{a}$ is finitely generated as an $R_{k}$-module. Elementary dimension theory for commutative rings then shows that

$$
\operatorname{kdim} R_{d} / \mathfrak{a} \leq \operatorname{kdim} R_{k}=k+1,
$$

from which we obtain the first inequality in (7-1).

To derive the second inequality in (7-1), start with an arbitrary minimal prime ideal $\mathfrak{p}$ over $\mathfrak{a}$. Krull's generalized principal ideal theorem [K, Thm. 152] shows that a maximal chain of prime ideals from $\{0\}$ to $\mathfrak{p}$ has length at most $g$. This chain can be extended to one of length $d+1$ [K, p. 114], proving that $\mathrm{kdim} R_{d} / \mathfrak{a} \geq d+1-g$. Putting this together, we have

$$
\operatorname{dim} V \geq \operatorname{kdim} R_{d} / \mathfrak{a}-1 \geq d-g .
$$

This proves $(7-1)$.

If one of the generators of $\mathfrak{a}$ is a rational prime $p$, then we can replace $R_{k}$ by $(\mathbb{Z} / p \mathbb{Z})\left[v_{1}^{ \pm 1}, \ldots, v_{k}^{ \pm 1}\right]$, which has Krull dimension $k$ rather than $k+1$. The same arguments then give the strengthened inequality (7-2).

Although (7-2) is sharp in the cases considered in Theorem 7.3, in general the inequalities can be strict. For example, let $f \in R_{d}$ have a zero $\left(z_{1}, \ldots, z_{d}\right) \in \mathbb{C}^{d}$ with $\left|z_{j}\right|=1$ for all $j$. Then by [S1, Thm. 3.9], $\alpha_{R_{d} /\langle f\rangle}$ is not expansive. Thus $\operatorname{erk}\left(\alpha_{R_{d} /\langle f\rangle}\right)=d+1$, while $d-g=d-1$.

\section{MARKOV SUBDYNAMICS}

In this section we consider the 1-dimensional subdynamics of a $\mathbb{Z}^{d}$-action, focusing on those properties involving stable sets. The basic approach is to define a version of the property for 1 -frames (i.e., vectors) $\mathbf{v}$ implying the standard version for $\alpha^{\mathbf{v}}$ when $\mathbf{v} \in \mathbb{Z}^{d}$, and then to show that the general version holds locally.

We first discuss stable foliations for maps. Let us say that two homeomorphisms $S$ and $T$ of a compact metric space $(X, \rho)$ have the same stable foliation if, for all $x, y \in X$,

$$
\lim _{n \rightarrow \infty} \rho\left(S^{n} x, S^{n} y\right)=0 \Longleftrightarrow \lim _{n \rightarrow \infty} \rho\left(T^{n} x, T^{n} y\right)=0 .
$$

Recall that $B(R)$ denotes the closed ball in $\mathbb{R}^{d}$ of radius $R$, and $E^{t}$ is the set of vectors in $\mathbb{R}^{d}$ within $t$ of $E$. For $\mathbf{v} \in \mathbb{R}^{d}$ we let $H_{\mathbf{v}}=[0, \infty) \mathbf{v}$ denote the half-line through $\mathbf{v}$. For $\theta>0$ let $K_{\theta}(\mathbf{v})$ denote the open cone of nonzero vectors making angle less that $\theta$ with $\mathbf{v}$. By an expansive radius for $\mathbf{v}$ we mean an expansive radius for $\mathbb{R} \mathbf{v}$ in the sense of Definition 2.4. A uniform expansive radius for a set in $\mathbb{R}^{d}$ is a number that is simultaneously an expansive radius for every element of the set.

Proposition 8.1. Suppose that $\mathcal{C}$ is an expansive component of 1-frames for a $\mathbb{Z}^{d}$-action $\alpha$.

(1) Let $\mathbf{u} \in \mathcal{C}$. Then there are $\theta>0, t>0$, and $R>0$ such that for all $\mathbf{v}, \mathbf{w} \in K_{\theta}(\mathbf{u})$, the set $H_{\mathbf{v}}^{t}$ codes $K_{2 \theta}(\mathbf{w})$, and the set $B(R) \cup H_{\mathbf{v}}^{t}$ codes $H_{\mathbf{w}}^{t}$. 
(2) Let $E$ be a compact subset of $\mathcal{C}$. Then $E$ has a uniform expansive radius. For each uniform expansive radius $\tau$ for $E$, there is an $R>0$ such that $B(R) \cup H_{\mathbf{v}}^{\tau}$ codes $B(R) \cup H_{\mathbf{w}}^{\tau}$ for all $\mathbf{v}, \mathbf{w} \in E$.

(3) All the maps $\alpha^{\mathbf{n}}, \mathbf{n} \in \mathcal{C} \cap \mathbb{Z}^{d}$, have the same stable foliation.

Proof. (1) Pick $\gamma$ small enough so that $\overline{K_{\gamma}(\mathbf{u})} \subset \mathcal{C}$, and let $\mathcal{K}$ be the corresponding compact set of lines in $\mathbb{G}_{1}$. For this $\mathcal{K}$, let $t>0$ and $r>0$ be the numbers provided by Proposition 3.8, and let $\beta=\sin ^{-1}(1 / r)$. Then for every $\mathbf{v} \in K_{\gamma}(\mathbf{u})$, the set $H_{\mathbf{v}}^{t}$ codes $K_{\beta}(\mathbf{v})$. Thus the first claim in (1) holds with $\theta=\beta / 3$. We can choose $R$ for the second claim because $K_{2 \theta}(\mathbf{w})$ contains all but a bounded subset of $H_{\mathbf{w}}^{t}$.

(2) If $\tau$ and $\tau^{\prime}$ are two uniform expansive radii for $E$, then there is an $R$ such that for all $\mathbf{u} \in E, B(R) \cup H_{\mathbf{u}}^{\tau}$ codes $H_{\mathbf{u}}^{\tau^{\prime}}$. Hence it suffices to prove (2) for a single uniform expansive radius for $E$.

Choose a compact set $F$ with $E \subset F \subset \mathcal{C}$ such that $F$ is the closure of a connected union of finitely many open balls that cover $E$. The number $t$ provided by Proposition 3.8 is a uniform expansive radius for $F$, hence for $E$. Thus we need only prove (2) for $F$ in place of $E$, and for the uniform expansive radius $t$.

By our choice of $t$, we can cover $F$ with open sets $K_{\theta_{i}}\left(\mathbf{u}_{i}\right), 1 \leq i \leq M$, satisfying (1) with numbers $R_{i}$ and the expansive radius $t$. Let $R=\max _{i} R_{i}$. Suppose that $\mathbf{x} \in K_{\theta_{i}}\left(\mathbf{u}_{i}\right), \mathbf{y} \in K_{\theta_{j}}\left(\mathbf{u}_{j}\right)$, and $\mathbf{z} \in K_{\theta_{i}}\left(\mathbf{u}_{i}\right) \cap K_{\theta_{j}}\left(\mathbf{u}_{j}\right)$. Then $B(R) \cup H_{\mathbf{x}}^{t}$ codes $B(R) \cup H_{\mathbf{z}}^{t}$, and $B(R) \cup H_{\mathbf{z}}^{t} \operatorname{codes} B(R) \cup H_{\mathbf{y}}^{t}$. For arbitrary $\mathbf{u}, \mathbf{v} \in F$, there is a finite chain of $K_{\theta_{i}}\left(\mathbf{u}_{i}\right)$ whose first set contains $\mathbf{u}$, whose last set contains $\mathbf{v}$, and for which each pair of successive sets has nonempty intersection. Repeated application of the previous argument then gives (2).

(3) Suppose that $\mathbf{n} \in \mathcal{C} \cap \mathbb{Z}^{d}$. Then $x, y \in X$ are in the same stable set for $\alpha^{\mathbf{n}}$ if and only if there is an $a>0$ such that

$$
\rho_{\alpha}^{([a, \infty) \mathbf{n})^{t}}(x, y) \leq \delta .
$$

Let $\theta, t$, and $R$ be provided by (1) for $\mathbf{u}=\mathbf{n}$. Let $\mathbf{m} \in K_{\theta}(\mathbf{u}) \cap \mathbb{Z}^{d}$. Chose $b>0$ so that $([-b, b] \mathbf{n})^{t}$ codes $B(R)$. Then $([a, \infty) \mathbf{n})^{t}=(a+b) \mathbf{n}+([-b, \infty) \mathbf{n})^{t}$, which codes $(a+b) \mathbf{n}+B(R)$. Hence $([a, \infty) \mathbf{n})^{t} \operatorname{codes}(a+b) \mathbf{n}+K_{2 \theta}(\mathbf{n})$, which contains all but a compact subset of $K_{\theta}(\mathbf{m})$. In particular, there is a $c>0$ such that $([a, \infty) \mathbf{n})^{t}$ codes $([c, \infty) \mathbf{m})^{t}$. This proves that the stable foliations of $\mathbf{m}$ and $\mathbf{n}$ are the same. Thus the stable foliation is locally constant, hence constant on $\mathcal{C}$ since $\mathcal{C}$ is connected.

We next define a Markov direction, and this requires some notation. For $\mathbf{v} \in \mathbb{R}^{d}$, let $B\left(t, \mathbf{v}^{\perp}\right)$ denote the closed ball of radius $t$ in the orthogonal complement of $\mathbf{v}$ in $\mathbb{R}^{d}$. We put

$$
\begin{aligned}
& H_{\mathbf{v}}^{+}(t, r)=[-r, \infty) \mathbf{v} \oplus B\left(t, \mathbf{v}^{\perp}\right), \\
& H_{\mathbf{v}}^{-}(t, r)=(-\infty, r] \mathbf{v} \oplus B\left(t, \mathbf{v}^{\perp}\right),
\end{aligned}
$$

which are overlapping semi-infinite "tubes" of radius $\tau$ surrounding $\mathbb{R} \mathbf{v}$.

Definition 8.2. Let $\mathbf{v}$ be an expansive vector, and $V=\mathbb{R} \mathbf{v}$. Then $\mathbf{v}$ (or $V$ ) is Markov if there is an $r>0$ and an expansive radius $t$ for $V$ such that whenever $x, y \in X$ with $\rho_{\alpha}^{V^{t}(r)}(x, y) \leq \delta$, then there is a $z \in X$ such that

$$
\rho_{\alpha}^{H_{\mathbf{v}}^{-}(t, r)}(x, z) \leq \delta \quad \text { and } \quad \rho_{\alpha}^{H_{\mathbf{v}}^{+}(t, r)}(y, z) \leq \delta .
$$

Roughly speaking, this definition says that if two points agree on the overlap $V^{t}(r)$ on the future $H_{\mathbf{v}}^{+}(t, r)$ and the past $H_{\mathbf{v}}^{-}(t, r)$, then the past of one and the 
future of the other can be pasted together to form a new point. When $\mathbf{v} \in \mathbb{Z}^{d}$, this definition coincides with the usual one for $\alpha^{\mathbf{v}}$ using canonical coordinates.

Proposition 8.3. Let $\mathcal{C}$ be an expansive component of 1-frames for a $\mathbb{Z}^{d}$-action. Then either every vector in $\mathcal{C}$ is Markov or no vector in $\mathcal{C}$ is Markov.

Proof. Suppose that $\mathbf{v}, \mathbf{w} \in \mathcal{C}$, and that $\mathbf{v}$ is Markov. Let $r, t$ be as in Definition 8.2. Increase $t$ so that it is also an expansive radius for $\mathbf{w}$. Then $\mathbf{v}$ is also Markov for this larger $t$ and all sufficiently large $R$. Choose $R>r$ satisfying Proposition 8.1(2) for the set $\{\mathbf{v}, \mathbf{w}\}$ and also for $\{-\mathbf{v},-\mathbf{w}\}$. Let $W=\mathbb{R} \mathbf{w}$. Choose $s$ such that $W^{t}(s)$ codes $B(R)$. We claim that $\mathbf{w}$ is Markov using $s$ and $t$. For if $\rho_{\alpha}^{W^{t}(s)}(x, y) \leq \delta$, then $\rho_{\alpha}^{B(R)}(x, y) \leq \delta$, so $\rho_{\alpha}^{V^{t}(r)}(x, y) \leq \delta$. Choose $z$ as in Definition 8.2 for $\mathbf{v}$. The coding relation from Proposition 8.1(2) shows that this same $z$ also works for $\mathbf{w}$.

Using this proposition, we may define an expansive component of 1-frames to be Markov provided that some (hence all) of its elements are Markov. If one integral vector in a Markov component $\mathcal{C}$ is mixing, then all elements of $\mathcal{C} \cap \mathbb{Z}^{d}$ are also mixing, since a shift of finite type commuting with a mixing shift of finite type must be mixing. We will refer to a component that contains a mixing Markov integral vector as a mixing Markov component.

The next result follows from Corollary 8.7 below, and can also be proved using results of Nasu [N2]. We give a simple geometric argument adapted from [Mi, p. 383].

Corollary 8.4. If $\mathcal{C}$ is a mixing Markov component of 1-frames, then $h_{1}$ is linear on $\mathcal{C}$.

Proof. First note that $\mathcal{C}$ is open, and convex by Remark 4.6. Since $h_{1}$ is continuous on $\mathcal{C}$ and rational lines are dense in $\mathcal{C}$, it suffices to prove that $h_{1}(\mathbf{v}+\mathbf{w})=h_{1}(\mathbf{v})+$ $h_{1}(\mathbf{w})$ for all $\mathbf{v}, \mathbf{w} \in \mathcal{C} \cap \mathbb{Z}^{d}$.

Recall that $Q_{\mathbf{v}}$ denotes $[\mathbf{0}, \mathbf{v}]$. As in the proof of Theorem 6.9, the set $Q_{\mathbf{v}} \cup$ $\left(\mathbf{v}+Q_{\mathbf{w}}\right)$ shades $Q_{\mathbf{v}+\mathbf{w}}$. By Lemma $6.28, Q_{\mathbf{v}+\mathbf{w}}$ shades $Q_{\mathbf{v}}$, and by symmetry also $\mathbf{v}+Q_{\mathbf{w}}$. Therefore the sets $Q_{\mathbf{v}+\mathbf{w}}$ and $Q_{\mathbf{v}} \cup\left(\mathbf{v}+Q_{\mathbf{w}}\right)$ shade each other, so that

$$
h_{1}(\mathbf{v}+\mathbf{w})=\eta_{1}\left(Q_{\mathbf{v}+\mathbf{w}}\right)=\eta_{1}\left(Q_{\mathbf{v}} \cup\left(\mathbf{v}+Q_{\mathbf{w}}\right)\right) .
$$

Thus it is enough to show that

$$
\eta_{1}\left(Q_{\mathbf{v}} \cup\left(\mathbf{v}+Q_{\mathbf{w}}\right)\right)=\eta_{1}\left(Q_{\mathbf{v}}\right)+\eta_{1}\left(Q_{\mathbf{w}}\right)=h_{1}(\mathbf{v})=h_{1}(\mathbf{w}) .
$$

Translating by $-\mathbf{v}$, we may just as well consider $\eta_{1}\left(Q_{-\mathbf{v}} \cup Q_{\mathbf{w}}\right)$.

Let $t$ be large, and fix $R$ for the set $\{\mathbf{v}, \mathbf{w}\}$ as in Proposition 8.1(2). For $x \in X$ and $\mathbf{u} \in \mathbb{R}^{d}$, let $x[\mathbf{u}]$ be the restriction of $x$ to $H_{\mathbf{u}}^{t} \cap \mathbb{Z}^{d}$, and $x(R)$ be the restriction of $x$ to $B(R)$. Then $x[-\mathbf{u}]$ and $x(R)$ determine $x[-\mathbf{v}]$ and $x(R)$, and vice versa. Because $\alpha^{\mathbf{v}}$ is mixing Markov, there is a transition length $N$ such that for every $y \in X$ there is a $z \in X$ such that $z(R)=x(R), z[-\mathbf{w}]=x[-\mathbf{w}]$, and $z(\mathbf{n})=y(\mathbf{n})$ for all $\mathbf{n} \in([N, \infty) \mathbf{w})^{t}$. Hence every configuration in $H_{-\mathbf{v}}^{t}$ occurs with every configuration in $N \mathbf{w}+H_{\mathbf{w}}^{t}$. Thus $\eta_{1}\left(Q_{-\mathbf{v}} \cup Q_{\mathbf{w}}\right)=\eta_{1}\left(Q_{-\mathbf{v}}\right)+\eta_{1}\left(Q_{\mathbf{w}}\right)$, as required.

The independence of configurations resulting from the Markov properties lies a the heart of this proof. This independence fails in the construction of examples with nonlinear $h_{1}$ from Theorem 6.33.

Corollary 8.5. Let $\mathcal{C}$ be a mixing Markov component of 1-frames, and $\mathbf{m}, \mathbf{n} \in$ $\mathcal{C} \cap \mathbb{Z}^{d}$. Then $\alpha^{\mathbf{m}}$ and $\alpha^{\mathbf{n}}$ have the same unique measure of maximal entropy. 
Proof. By Corollary 8.4, $h_{1}$ is linear on $\mathcal{C}$. Then Theorem 6.25 shows that $\alpha^{\mathbf{m}}$ and $\alpha^{\mathbf{n}}$ have a common measure of maximal entropy. Since both $\alpha^{\mathbf{m}}$ and $\alpha^{\mathbf{n}}$ are mixing Markov, this measure is unique.

The next result deals with the dimension group of a Markov shift, and assumes familiarity with, say, [BMT]. For an $n \times n$ integral matrix $A$, let

$$
\Delta_{A}=\left\{\mathbf{v} \in A^{n}\left(\mathbb{Q}^{n}\right): A^{k} \mathbf{v} \in \mathbb{Z}^{d} \text { for all large } k\right\},
$$

and $\delta_{A}$ denote the automorphism of $\Delta_{A}$ induced by restriction of $A$. Then $\left(\Delta_{A}, \delta_{A}\right)$ is called the dimension pair of $A$. If two mixing Markov shifts are defined by matrices $A$ and $B$, then they are shift equivalent if and only if they have isomorphic dimension pairs, i.e., there is an isomorphism $\theta: \Delta_{A} \rightarrow \Delta_{B}$ such that $\theta \delta_{a}=\delta_{B} \theta$. The group $\Delta_{A}$ is a presentation of the dimension group of $A$, which was defined intrinsically by Krieger (see $[\mathrm{Kr} 1],[\mathrm{Kr} 2],[\mathrm{BK}],[\mathrm{BMT}]$ ). Dimension groups are ordered, and when $A$ is mixing the order is easily determined from the dominant (or Perron) eigenvalue and its Perron eigenline.

In the following, if $\mathbf{a}=\left(a_{1}, \ldots, a_{d}\right)$ is a $d$-tuple of complex numbers and $\mathbf{n} \in \mathbb{Z}^{d}$, put $\mathbf{a}^{\mathbf{n}}=a_{1}^{n_{1}} a_{2}^{n_{2}} \ldots a_{d}^{n_{d}}$. Similarly, if $\mathbf{B}=\left(B_{1}, \ldots, B_{d}\right)$ is a $d$-tuple of matrices, put $\mathbf{B}^{\mathbf{n}}=B_{1}^{n_{1}} B_{2}^{n_{2}} \ldots B_{d}^{n_{d}}$.

Theorem 8.6. Let $\mathcal{C}$ be a mixing Markov component of 1-frames for $\alpha$. Then there is a group $\Delta$ and a d-tuple $\mathbf{B}=\left(B_{1}, \ldots, B_{d}\right)$ of nonsingular commuting rational matrices such that for all $\mathbf{n} \in \mathcal{C} \cap \mathbb{Z}^{d}$ the dimension pair of $\alpha^{\mathbf{n}}$ is $\left(\Delta, \mathbf{B}^{\mathbf{n}}\right)$. The $\mathbf{B}^{\mathbf{n}}$ for $\mathbf{n} \in \mathcal{C} \cap \mathbb{Z}^{d}$ all have a common Perron eigenline.

Proof. By Proposition 8.1, all $\alpha^{\mathbf{n}}$ with $\mathbf{n} \in \mathcal{C} \cap \mathbb{Z}^{d}$ have the same stable foliation. Then the argument in [BK, Thm. 2.17] shows that they have the same ordered dimension group $\left(\Delta, \Delta^{+}\right)$, and give rise to a commuting system of order-preserving automorphisms, which can clearly be realized by rational matrices. Because the ordered group does not change, these matrices must have the same Perron eigenline.

We do not know whether we can arrange that the matrices $\mathbf{B}^{\mathbf{n}}$ in this theorem are integral.

Corollary 8.7. Let $\mathcal{C}$ be a mixing Markov component of 1-frames for a $\mathbb{Z}^{d}$-action $\alpha$. Then there are d-tuples $\boldsymbol{\Theta}_{1}=\left(\theta_{11}, \ldots, \theta_{1 d}\right), \ldots, \boldsymbol{\Theta}_{r}=\left(\theta_{r 1}, \ldots, \theta_{r d}\right)$ of algebraic numbers such that for all $\mathbf{n} \in \mathcal{C} \cap \mathbb{Z}^{d}$ the zeta function $\zeta_{\mathbf{n}}(z)$ of $\alpha^{\mathbf{n}}$ is given by

$$
\zeta_{\mathbf{n}}(z)=\frac{1}{\prod_{j=1}^{r}\left(1-\mathbf{\Theta}_{j}^{\mathbf{n}} z\right)} .
$$

Proof. If the dimension automorphism of $\alpha^{\mathbf{n}}$ is induced by a matrix $B$ with eigenvalues $\lambda_{1}, \ldots, \lambda_{r}$, then $\zeta_{\mathbf{n}}(z)=1 / \prod_{j=1}^{r}\left(1-\lambda_{j} z\right)$. The result now follows from Theorem 8.6.

Remark 8.8. For $\mathbb{Z}^{d}$-actions on a zero-dimensional space, one can give an analogue of Definition 8.2 for the sofic property in a direction $\mathbf{v}$ using follower sets, such that if $\mathbf{v} \in \mathbb{Z}^{d}$ this is the same as $\alpha^{\mathbf{v}}$ being a sofic homeomorphism. The analogue of Proposition 8.3 holds, so that in an expansive component either all elements are sofic or none are. 
Remark 8.9. For a rational Markov or sofic direction of a $\mathbb{Z}^{2}$-action, the local analysis of this section could be done using Nasu's textile system machinery [N2]. This provides finer detail and constructive matrix tools. The difficulty is that without defining properties on a completed space of directions, it is difficult to show how the local analysis gives results valid over an entire expansive component.

Remark 8.10. There is an analogous definition of Markov for $k$-planes. Let $V$ be an expansive $k$-plane, and $L$ be a $(k-1)$-dimensional subspace of $V$. Let $V^{+}$ and $V^{-}$denote the two half-planes determined by $L$. Say that $V$ is $L$-Markov if there are $R>0$ and an expansive radius $t$ to $V$ such that whenever $x, y \in X$ with $\rho_{\alpha}^{L^{R}}(x, y) \leq \delta$, then there is a $z \in X$ such that

$$
\rho_{\alpha}^{\left(V^{-}\right)^{t}}(x, z) \leq \delta \quad \text { and } \quad \rho_{\alpha}^{\left(V^{+}\right)^{t}}(y, z) \leq \delta .
$$

Then the higher dimensional analogue of Proposition 8.1 is true. This can be used to prove that for a fixed $(k-1)$-plane $L$, within a connected component of expansive $k$-planes containing $L$, either all are $L$-Markov or none are.

\section{Problems}

Our analysis of expansive subdynamics provides a framework for investigating $\mathbb{Z}^{d}$-actions, and suggests a number of further problems and research directions.

Structure. By Theorem 3.7, $\mathbb{N}_{d-1}(\alpha)$ determines all the other $\mathbb{N}_{k}(\alpha)$. We do not know a complete description of what $\mathbb{N}_{d-1}(\alpha)$ can look like.

Problem 9.1. Which closed sets of $\mathbb{G}_{d-1}$ are $\mathbb{N}_{d-1}(\alpha)$ for some $\mathbb{Z}^{d}$-action $\alpha$ on a compact metric space? Does the answer change if we require $\alpha$ to be topologically mixing?

Theorem 4.3 provides a nearly complete answer when $d=2$. However, it leaves open one case that has resisted strenuous efforts.

Problem 9.2. Given an irrational line $L$ in $\mathbb{R}^{2}$, is there a $\mathbb{Z}^{2}$-action $\alpha$ for which $\mathbb{N}_{1}(\alpha)=\{L\} ?$

There is what appears to be a related question about when $\mathbb{N}_{1}(\alpha)$ consists of a single rational line.

Problem 9.3. Suppose that $\alpha$ is a $\mathbb{Z}^{2}$-action for which $\mathbb{N}_{1}(\alpha)=\{L\}$, where $L$ is a rational line through $\mathbf{n} \in \mathbb{Z}^{2}$. Must there be a $k \geq 1$ for which $\alpha^{k \mathbf{n}}$ is the identity map?

With Markov assumptions, these sorts of problems take on a different flavor.

Problem 9.4. Which closed sets in $\mathbb{G}_{d-1}$ are $\mathbb{N}_{d-1}(\alpha)$ for a Markov $\mathbb{Z}^{d}$-action $\alpha$ ?

Note that there are only countably many possibilities for Markov $\mathbb{Z}^{d}$-actions (up to conjugacy), so the class of closed sets here is much more restricted.

Problem 9.5. If $\alpha$ is a $\mathbb{Z}^{2}$-action with a Markov direction, must $\mathbb{E}_{1}(\alpha)$ be a finite union of intervals with rational endpoints?

Compatibility of component subdynamics. How does certain dynamical behavior in one expansive component influence behavior in other components?

Problem 9.6. If one expansive component of $\mathbb{E}_{1}(\alpha)$ is Markov, must all other expansive components be Markov? 
This can be recast as the following problem due to Nasu [N2], which arises when deciding whether certain algorithms in [N2] terminate.

Problem 9.7. If an expansive homeomorphism commutes with a shift of finite type, must it also be of finite type?

Theorem 8.6 shows that associated to each Markov component is a dimension group. Work of Nasu [N2] shows that the dimension groups of different Markov components need not be isomorphic.

Problem 9.8. Which pairs of groups can arise as the dimension groups of different Markov components of a $\mathbb{Z}^{2}$-action?

The next problem asks whether a pair of mixing shifts of finite type can be embedded in a $\mathbb{Z}^{2}$-action.

Problem 9.9. Given two mixing shifts of finite type $S$ and $T$, is there a $\mathbb{Z}^{2}$-action $\alpha$ for which $\alpha^{\mathbf{e}_{1}}$ is topologically conjugate to $S$ and $\alpha^{\mathbf{e}_{2}}$ is topologically conjugate to $T$ ?

Observe that for this problem there are some obstructions involving periodic point counts, since the set of points with $S$-period $n$ is $T$-invariant.

Entropy. There are a number of unresolved problems concerning the "entropy geometry" of actions and directional entropy. The first is about the information function $\eta_{k}$, defined for all compact subsets of $\mathbb{R}^{d}$.

Problem 9.10. Let $\alpha$ be an algebraic action (see $\S 7$ ) having expansive rank $k$. Compute $\eta_{k}(E)$ for all compact sets $E \subset \mathbb{R}^{d}$. In particular, if $V$ is a $k$-plane, is $\eta_{k}(E)=h_{k}(V) \lambda_{V}(E)$ for all compact $E \subset V$ ? Note that according to Theorem 6.3, this is true for all $E$ with $\lambda_{V}(\partial E)=0$.

Problem 9.11. Understand the shading relation among compact sets (see Definition 6.7), and its relation to expansiveness. For which compact sets $E$ is there a unique maximal closed set shaded by $E$ (which would naturally be called the "shadow" of $E)$ ?

Continuity properties of directional entropy are still obscure. Work of Sinai [Si1], which contains an inaccuracy corrected by Park [P], shows that if $\alpha$ is a $\mathbb{Z}^{2}$-action on a zero-dimensional space containing a Markov direction, then $h_{1}^{\mu}$ is upper semicontinuous. A detailed account of this is given in Lecture 8 of [Si2].

Problem 9.12. Suppose that $\alpha$ is a $\mathbb{Z}^{d}$-action having an expansive 1-dimensional line. Must $h_{1}$ be continuous? Must $h_{1}^{\mu}$ be continuous for every $\alpha$-invariant measure $\mu$ ?

Regularity on components. How regular is dynamical behavior on an expansive component?

Problem 9.13. Is there always a uniform expansive radius for an expansive component?

Problem 9.14. If $\mathbf{m}$ and $\mathbf{n}$ are integral vectors in the same expansive component for $\alpha$, and if $\alpha^{\mathbf{m}}$ is mixing, must $\alpha^{\mathbf{n}}$ also be mixing?

Transitional subdynamics. We know very little that constrains the subdynamics of an action in the nonexpansive directions. Here is a sample problem. 
Problem 9.15. Suppose that $\alpha$ is a $\mathbb{Z}^{2}$-action having a Markov direction. Must $h_{1}$ be piecewise linear on a closed interval of nonexpansive directions separating a pair of expansive components?

One can check using skew products and topological pressure that this is true for the class of $\mathbb{Z}^{2}$-actions generated by automorphisms of a shift of finite type studied in $[\mathrm{L}]$.

Other parameter groups. In our study of the subdynamics of $\mathbb{Z}^{d}$-actions, "completing" the space of directions plays a crucial role. A further step is to "complete" the acting group $\mathbb{Z}^{d}$ by taking the $\mathbb{R}^{d}$ constant-time suspension, so that now every direction corresponds to an element of the acting group. Katok and Spatzier [KaSp] did exactly this in their fruitful analysis of jointly invariant measures for certain geometric $\mathbb{Z}^{d}$-actions.

An open problem is to develop a topological dynamical theory of "expansive subdynamics" for an expansive $\mathbb{R}^{d}$-action on a compact metric space. One requirement of such a theory is that theorems about the subdynamics of $\mathbb{Z}^{d}$-actions should correspond to theorems about the subdynamics of their $\mathbb{R}^{d}$-suspensions. Some care is needed when defining expansiveness for a subaction (see [BW]). To make this development worth the effort, there should be adequate motivating examples of expansive $\mathbb{R}^{d}$-actions that are not suspensions of $\mathbb{Z}^{d}$-actions.

Finally, we have been considering the lattice $\mathbb{Z}^{d}$ in $\mathbb{R}^{d}$, and considering the subdynamics of closed subgroups in $\mathbb{R}^{d}$. What generalizes to lattices in Lie groups?

\section{REFERENCES}

[AKM] R. L. Adler, A. G. Konheim and M. H. McAndrew, Topological entropy, Trans. Amer. Math. Soc. 114 (1965) 309-319. MR 30:5291

[A] N. Aoki, Topological dynamics, in Topics in General Topology, North-Holland, Amsterdam (1989) 625-740. MR 91m:58120

[AM] N. Aoki and K. Moriyasu, Expansive homeomorphisms of solenoidal groups Hokkaido Math. J. 18 (1989), 301-319. MR 90i:58148

[BW] R. E. Bowen and P. Walters, Expansive one-parameter flows, J. Diff. Equations 12 (1972), 180-193. MR 49:6202

[BK] M. Boyle and W. Krieger, Periodic points and automorphisms of the shift, Trans. Amer. Math. Soc. 302 (1987), 125-149. MR 88g:54065

[BLR] M. Boyle, D. Lind, and D. Rudolph, The automorphism group of a shift of finite type, Trans. Amer. Math. Soc. 306 (1988), 71-114. MR 89m:54051

[BMT] M. Boyle, B. Marcus and P. Trow, Resolving maps and the dimension group for shifts of finite type, Memoirs of the Amer. Math. Soc. 377 (1987). MR 89c:28019

[DGS] M. Denker, C. Grillenberger and K. Sigmund, Ergodic Theory on Compact Spaces in Springer Lecture Notes in Math 527, Springer-Verlag, (1976). MR 56:15879

[Fa] A. Fathi, Expansiveness, hyperbolicity and Hausdorff dimension, Commun. Math. Phys. 126 (1989), 249-262. MR 90m:58159

[Fr1] D. Fried, Metriques naturelles sur les espaces de Smale, C. R. Acad. Sc. Paris 297 (1983), 77-79. MR 85c:58085

[Fr2] D. Fried, Entropy for smooth abelian actions, Proc. Amer. Math. Soc. 87 (1983), 111-117. MR 83m:54078

[Fr3] D. Fried, Rationality for isolated expansive sets, Advances in Math. 65 (1987), 35-38. MR 88i:58144

[Fr4] D. Fried, Finitely presented dynamical systems, Ergod. Th. E3 Dyn. Syst. 7 (1987), 489-507. MR 89h:58157

[FR] D. B. Fuks and V. A. Rokhlin, Beginner's Course in Topology, Springer-Verlag, New York, (1984). MR 86a:57001

[Go] L. W. Goodwyn, Some counterexamples in topological entropy, Topology 11 (1972), 377-385. MR 47:2575 
[GH] W. Gottschalk and G. Hedlund, Topological Dynamics, AMS Colloq. Publ., 36 Providence (1955). MR 17:650e

[G] Branko Grünbaum, Convex Polytopes, Interscience, London (1967). MR 37:2085

[H1] K. Hiraide, Expansive homeomorphisms of compact surfaces are pseudo-Anosov, Osaka J. Math. 27 (1990), 117-162. MR 91b:58184

[H2] K. Hiraide, Dynamical systems of expansive maps on compact manifolds, Sugaku Expositions 5 (No.2) (1992), 133-154. MR 91d:58197

[IT] Sh. Ito and Y.Takahashi, Markov subshifts and realization of beta-expansions, J. Math. Soc. Japan 26 (1974), 33-55. MR 49:10860

[K] Irving Kaplansky, Commutative Rings, Univ. of Chicago Press, Chicago (1974). MR 49:10674

[Ka] H. Kato, Expansive homeomorphisms in continuum theory, Topology Appl. 45 (1992), 223-243. MR 93j:54023

[KaSp] A. Katok and R. J. Spatzier, Invariant measures for higher rank hyperbolic abelian actions, Ergod. Th. E Dyn. Syst., to appear.

[KR] K. H. Kim and F. W. Roush, Williams' conjecture is false for reducible subshifts, Jour. Amer. Math. Soc. 5 (1992), 213-215. MR 92j:54055

[KRW] K. H. Kim, F. W. Roush and J. B. Wagoner, Automorphisms of the dimension group and gyration numbers of automorphisms of the shift, Jour. Amer. Math. Soc. 5 (1992), 191-212. MR 93h:54026

[Ki] J. L. King, A map with topological minimal self joinings in the sense of del Junco, Erg. Th. E Dyn. Syst. 10 (1990), 745-761. MR 92a:54036

[KS1] B. Kitchens and K. Schmidt, Automorphisms of compact groups, Ergod. Th. E Dyn. Syst. 9 (1989), 691-735. MR 91g:22008

[KS2] B. Kitchens and K. Schmidt, Markov subgroups of $(\mathbb{Z} / 2 \mathbb{Z})^{\mathbb{Z}^{2}}$, in Symbolic Dynamics and its Applications, American Math. Soc., Providence (1992). MR 93k:58136

[Kr1] W. Krieger, On dimension functions and topological Markov chains, Inventiones Math. 56 (1980), 239-250. MR 81m:28018

[Kr2] W. Krieger, On a dimension for a class of homeomorphism groups, Math. Ann. 252 (1980), 87-95. MR 82b:46083

[La] W. Lawton, The structure of compact connected groups which admit an expansive automorphism, Springer Lecture Notes Math. 318 (1973), 182-196. MR 52:11873

[Led] F. Ledrappier, Un champ markovian peut être d'entropie nulle et mélangeant, $C$. $R$. Acad. Sci. Paris, Ser. A 287 (1978), 561-563. MR 80b:28030

[Lew] J. Lewowicz, Expansive homeomorphisms of surfaces Bull. Soc. Brasil Math. 20 (1989), 113-133. MR 92i:58139

[L] D. A. Lind, Entropies of automorphisms of a topological Markov shift, Proc. Amer. Math. Soc. 99 (1987), 589-595. MR 88c:54034

[LSW] D. Lind, K. Schmidt, and T. Ward, Mahler measure and entropy for commuting automorphisms of compact groups, Invent. Math. 101 (1990), 593-629. MR 92j:22013

[LW] D. Lind and T. Ward, Automorphisms of solenoids and p-adic entropy, Ergod. Th. \& Dynam. Sys. 8 (1988), 411-419. MR 90a:28031

[Man1] Ricardo Mañé, Expansive homeomorphisms and topological dimension, Trans. Amer. Math. Soc. 252 (1979), 313-319. MR 80i:58032

[Man2] Ricardo Mañé, Ergodic theory and differentiable dynamics, Springer-Verlag, (1987). MR 88c: 58040

[Mt] H. Matsumura, Commutative Ring Theory, Cambridge Univ. Press, Cambridge (1989). MR 90i:13001

[Mi] John Milnor, On the entropy geometry of cellular automata, Complex Systems 2 (1988), 357-386. MR 90c:54026

[Mis] M. Misiurewicz, A short proof of the variational principle for a $\mathbb{Z}_{+}^{N}$ action on a compact space, Asterisque 40 (1976), 147-187. MR 56:3250

[N1] M. Nasu, Lecture on textile systems, CBMS Conference on Symbolic Dynamics, University of Washington (1989).

[N2] M. Nasu, Textile systems for endomorphisms and automorphisms of the shift, Memoirs Amer. Math. Soc. 546 (1995). MR 95i:54051

[P] K. Park, Continuity of directional entropy, Osaka J. Math. 31 (1994), 613-628. MR 95m:28021 
[Pe] J. B. Pesin, Lyapunov characteristic exponents and smooth ergodic theory, Russian Math. Surveys 32 (1977), 55-114.

[R] W. Reddy, Expansive canonical coordinates are hyperbolic, Topology Appl. 15 (1983), 205-210. MR 84a:54076

[S1] K. Schmidt, Automorphisms of compact abelian groups and affine varieties, Proc. London Math. Soc. 61 (1990), 480-496. MR 91j:28015

[S2] K. Schmidt, Dynamical Systems of Algebraic Origin, Progress in Math., 128, Birkhäuser, 1995. CMP 95:16

[SW] K. Schmidt and T. Ward, Mixing automorphisms of compact groups and a theorem of Schlickewei, Invent. Math. 111 (1993), 69-76. MR 95c:22011

[Sh] M. Shereshevsky, Expansiveness, entropy and polynomial growth for groups acting on subshifts by automorphisms, Indag. Math. (2) 4 (1993), 203-210. MR 94c:54074

[Si1] Ya. Sinai, An answer to a question of J. Milnor, Comment. Math. Helv. 60 (1985), 173-178. MR 86m:28012

[Si2] Ya. Sinai, Topics in Ergodic Theory (1994), Princeton Univ. Press, Princeton, N.J. MR 95j: 28017

[Sma] S. Smale, Differentiable dynamical systems, Bull. Amer. Math. Soc. 73 (1967), 747-817. MR 37:3598

[Smi] J. Smillie, Properties of the directional entropy function for cellular automata, Springer Lecture Notes in Math 1342 (1988), 689-705. MR 90b:58150

[W] Peter Walters, An Introduction to Ergodic Theory, Springer-Verlag, New York (1982). MR 84e:28017

Department of Mathematics, University of Maryland, College Park, Maryland 20742

E-mail address: mmb@math.umd.edu

Department of Mathematics, University of Washington, Box 354350, Seattle, WashINGTON 98195-4350

E-mail address: lind@math.washington.edu 